\title{
Handhold-Mediated Strand Displacement: A Nucleic Acid-Based Mechanism for Generating Far-from-Equilibrium Assemblies Through Templated Reactions
}

\author{
Javier Cabello-Garcia + , Wooli Bae, Guy-Bart V. Stan and Thomas E. Ouldridge * $^{*}$ \\ Department of Bioengineering and Centre for Synthetic Biology, Imperial College London, SW7 2AZ London, U.K.
}

\begin{abstract}
The use of templates is a well-established method for the production of sequence-controlled assemblies, particularly long polymers. Templating is canonically envisioned as akin to a self-assembly process, wherein sequence-specific recognition interactions between a template and a pool of monomers favour the assembly of a particular polymer sequence at equilibrium. However, during the biogenesis of sequence-controlled polymers, template recognition interactions are transient; RNA and proteins detach spontaneously from their templates to perform their biological functions and allow template reuse. Breaking template recognition interactions puts the product sequence distribution far from equilibrium, since specific product formation can no longer rely on an equilibrium dominated by selective copy-template bonds. The rewards of engineering artificial polymer systems capable of spontaneously exhibiting non-equilibrium templating are large, but fields like DNA nanotechnology lack the requisite tools; the specificity and drive of conventional DNA reactions rely on product stability at equilibrium, sequestering any recognition interaction in products. Here we introduce handhold-mediated strand displacement (HMSD), a DNA-based reaction mechanism suited to producing out-ofequilibrium products. HMSD decouples the drive and specificity of the reaction by introducing a transient recognition interaction, the handhold. We measure the kinetics of 98 different HMSD systems to prove that handholds can accelerate displacement by four orders of magnitude without being sequestered in the final product. We then use HMSD to template the selective assembly of any one product DNA duplex from an ensemble of equally stable alternatives, generating a far-from-equilibrium output. HMSD thus brings DNA nanotechnology closer to the complexity of out-of-equilibrium biological systems.
\end{abstract}

KEYWORDS: reaction mechanism, self-assembly, fluorescence, nucleic acids, kinetics

Over the last 25 years, DNA has proven a versatile engineering material for developing synthetic molecular systems. Achievements have included self-assembled structures, information-processing architectures, and molecular motors. ${ }^{1-5}$ However, a class of processes essential to biology - the chemically-driven far-fromequilibrium templating - has been largely neglected.

Far-from-equilibrium templating underlies the processes that constitute the central dogma of molecular biology. ${ }^{6-8}$ In a templated process, a pool of monomers assembles into a product of a specific sequence by interacting with the sequence elements of a template. ${ }^{9}$ For example, during RNA transcription, ribonucleotides use sequence-specific recognition interactions with a DNA template to polymerise into a sequence-complementary RNA strand. ${ }^{6}$ The essence of templating is illustrated in the context of dimerization in Fig.1, where it is contrasted with conventional self-assembly.

Therefore, as in any templating process, the sequence of the product (the RNA) is determined solely by the information transmitted by the recognition interactions with the template, rather than by interactions within the product itself (the key determinant in conventional self-assembly, Fig.1-a). This information transferred by the template is essential to biological complexity. For example, there is simply not enough information stored in the interactions of amino acids alone to direct their assembly into tens of thousands of distinct proteins, and only those proteins. ${ }^{10}$

A crucial feature of natural templating processes is that the product must eventually detach from the template, breaking all recogni- tion interactions (Fig.1-c). Firstly, this decoupling allows the product to operate independently of the template - proteins would be extremely restricted if they were anchored to their template polynucleotides. Secondly, the decoupling of the product allows the template to be used repeatedly. Without template recovery, templates would not solve the mechanistic problem of directing the assembly of proteins, since a new template would need to be assembled de novo from its monomers for each protein produced.

It is product detachment the step that necessarily pushes the products of natural templating processes far from equilibrium. Before separation, templated processes can rely on highly specific recognition interactions between template and monomers to assemble the correct product at equilibrium (Fig.1-b). ${ }^{11}$ However, if interactions between template and product are transient, there can be no systematic equilibrium bias for a template-matching sequence in the final product, since no specific interactions remain to provide this bias (Fig.1-c). ${ }^{11,12}$ Sequence-matched products and templates are therefore necessarily far from equilibrium in this setting.

We emphasize that "far from equilibrium" in this sense does not mean the creation of steady states with fuel-consuming cycles. Rather, the reactants are directed by the templates into assemblies that are effectively long-lived kinetic traps. Template-favoured product sequences are formed faster, and the slow conversion into alternative sequences means that the system does not relax to equilibrium on accessible timescales. Unlike the unintentional kinetic traps that often impede reliable self-assembly, ${ }^{13}$ these kinetic traps are the required product. Moreover, each template sequence must reliably direct the reactants towards a distinct trapped state. 
a)

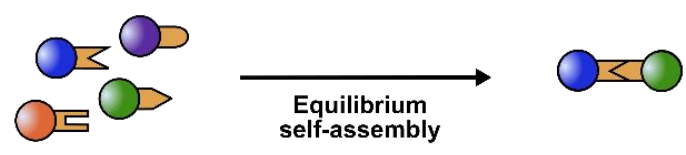

b)

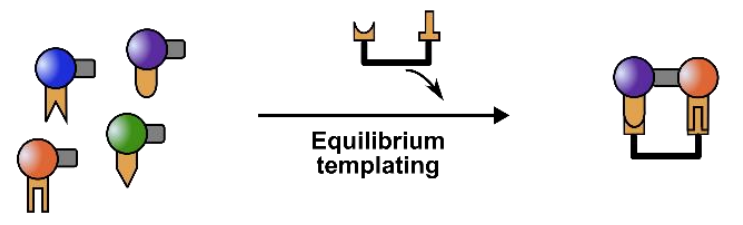

c)

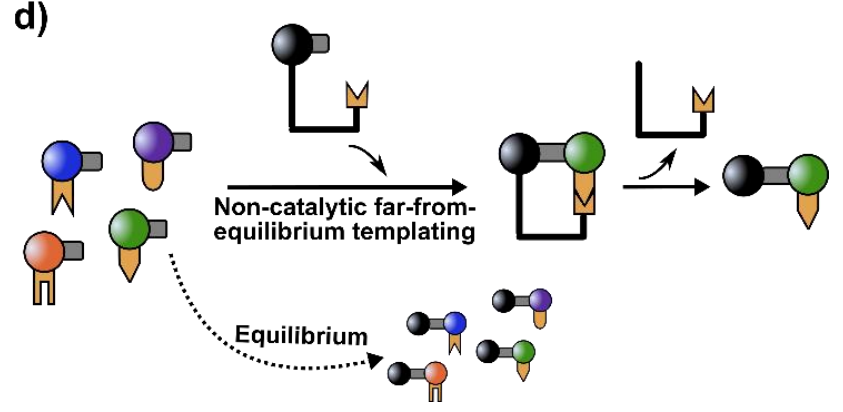

Figure 1: Dimer formation mechanisms. a) Equilibrium self-assembly. The introduction of complementary recognition interactions between monomers biases the formation of specific products at equilibrium. b) Equilibrium templating. Templates use complementary recognition interactions to determine the spatial organisation of the two interacting monomers. Once bound to the template, the monomers bind to each other through a generic bond. The template-complementary dimer is favoured at equilibrium due to its specific interactions with the template. c) Catalytic far-fromequilibrium templating. After the production of the generic bond between monomers, the dimer and template can detach spontaneously. The template can then be reused to catalyse again the formation of that dimer and push its concentration out-of-equilibrium. Since only the generic bond persists, all possible dimers are equally probable in the unwanted equilibrium ensemble. d) Non-catalytic far-from-equilibrium templating as implemented in this work. A process simpler than (c) in which templates are pre-loaded with one monomer, with the focus on the selective production and detachment of a dimer once the first monomer is bound. Although simpler, the products are still in a far-from equilibrium ensemble driven by transient recognition interactions with the template.

Specifically, the distribution $p(\boldsymbol{s})$ of product sequences, $\boldsymbol{s}$, must be sharply peaked around the complement of the template sequence $\boldsymbol{s}_{0}$. Even though the equilibrium distribution $p_{\mathrm{eq}}(\boldsymbol{s})$ is necessarily independent of $\boldsymbol{s}_{0}$, and essentially uniform. For a perfectly matching product of length $N$, and an alphabet of $M$ distinct monomers, the excess free energy stored in this non-equilibrium product distribution is approximately $N R T \ln M$ per mole of product. ${ }^{11}$

Given the fundamental significance of far-from-equilibrium templating, achieving similar functionality in synthetic systems would provide a platform for the development of more complex chemical and biomolecular systems. The success of DNA nanotechnology suggests that short, synthetic oligonucleotides may be the ideal engineering material for this challenge. ${ }^{14-16}$ Yet, it is far more challenging to engineer systems to reach these far-from-equilibrium target states than to design a system that relaxes to a specific structure at equilibrium. ${ }^{17}$ Moreover, the central reaction underlying dynamic DNA nanotechnology - toehold-mediated strand displacement (TMSD) - is poorly suited to non-equilibrium templating.

TMSD is a spontaneous reaction in which an invader nucleic acid strand $(I)$ replaces an incumbent strand $(N)$ in a duplex with a target strand (T) (Fig.2-a). ${ }^{18}$ TMSD is driven by toeholds, overhanging single-stranded domains in $T$ recognised by the $I$ strand. Toeholds increase TMSD reaction rates by inhibiting the detachment of the $I$ strand from $T$ during the competition with $N,{ }^{19}$ a process known as branch migration. Thus, TMSD reaction rates increase exponentially with toehold length until saturating at approximately six orders of magnitude faster than toehold-free displacement, at a toehold length of 6-7 nucleotides (nt).$^{20}$ Toehold versatility and ease of design have made TMSD a great building block for constructing an enormous range of chemical reaction networks. ${ }^{21,22}$

The toehold and the contiguous displacement domain act cooperatively to ensure the stability of the $I T$ complex. Toehold and displacement domain cooperativity remains even for more complex TMSD topologies - like associative ${ }^{23}$ (Fig.2-b) and remote ${ }^{24}$ toeholds - that locate the toeholds in positions that are non-contiguous with the displacement domain. The result is that toeholds - the recognition domains of strand displacement - are typically tightly sequestered in products after the reaction takes place. TMSD is therefore poorly suited to far-from-equilibrium templating, in which recognition interactions are transient interactions with a template, rather than permanent interactions incorporated into the product. As a result, attempts at far-from-equilibrium templating using TMSD are inherently limited.

Other nucleic acid-based approaches to templating that rely directly on hybridisation between DNA strands have faced related challenges. The tendency of assembled products to bind cooperatively to templates and "inhibit" their function ${ }^{25,26}$ restricts its output to the production of dimers. ${ }^{27}$ unless non-chemical mechanisms such as mechanical agitation, ${ }^{28}$ or cyclically varying conditions, are used to separate the product from the template. ${ }^{14-16}{ }^{29}$ However, these externally-driven approaches to reuse the template are much harder to implement as an autonomous reaction networks in general contexts. Other, non-nucleic-acid-based synthetic templated systems have faced similar challenges. ${ }^{30,31}$

Here, we introduce HMSD (Fig.2-c), a strand displacement topology capable of introducing far-from-equilibrium templating to DNA reaction networks. HMSD increases the rate of displacement of $N$ from a complex $N T$ through recognition of an overhanging "handhold" domain located in $N$, rather than a toehold in $T$. The molecular logic of recognising one molecule $N$ and binding to another $T$ is more naturally suited to templating than TMSD. Moreover, since the handhold is not cooperatively sequestered in the product $I T$, it can instead function as a transient bond with a template $N$. 
a)

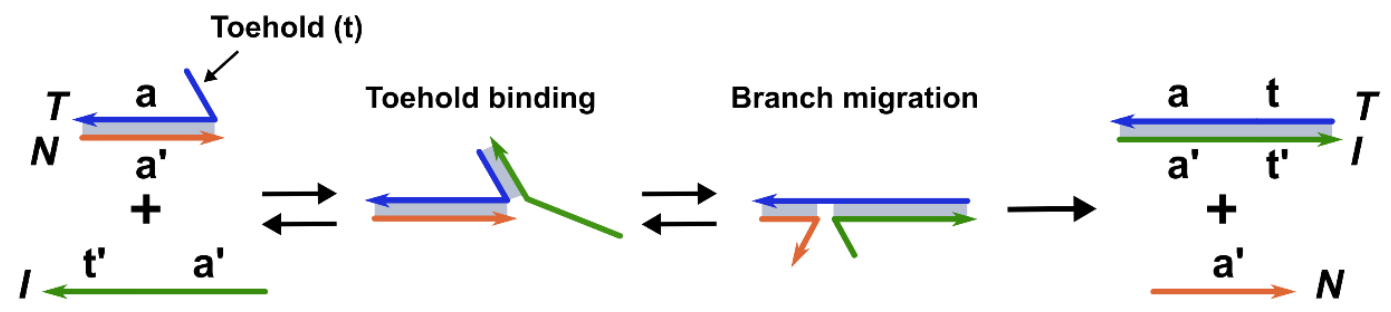

b)

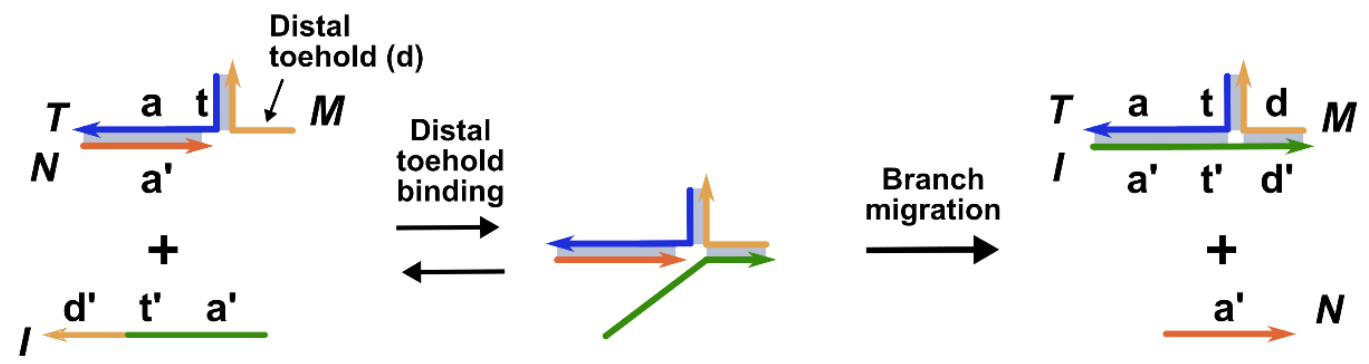

c)

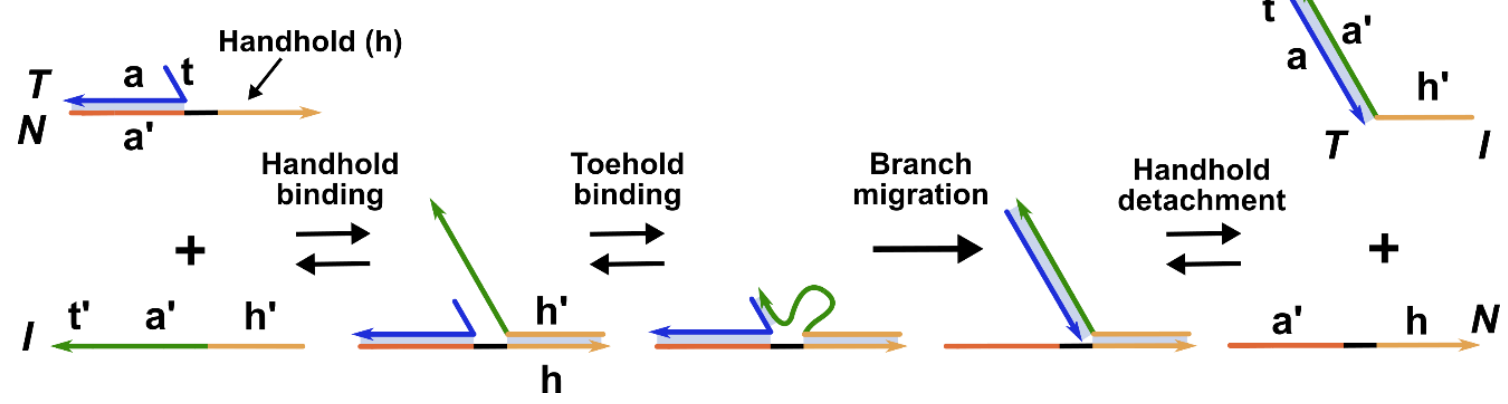

Figure 2: Strand displacement topologies. a) Toehold-mediated strand displacement. The presence of a proximal toehold (t) in the target strand $(T)$ mediates the displacement of the incumbent strand $(N)$ by the invader strand $(I)$. After displacement, $\mathrm{t}$ remains cooperatively sequestered in the product $I T$. b) Associative toehold. An auxiliary strand $(M)$, complementary to the strand $I$ and $T$, accelerates the displacement by effectively increasing the length of t. The reaction results in the formation of a stable three-stranded product $I T M$ that cooperatively sequesters the associative toehold. c) Handhold-mediated strand displacement (HMSD). The incorporation of a handhold (h) - an independent overhang in $N$-increases the effective concentration of the $I$ strand in the vicinity of $T$, enhancing the efficiency of displacement with short proximal toeholds. Reversible handhold binding for handholds of moderate length results in a displacement rate increase for handhold-complementary I strands, while still allowing the detachment of IT from short handholds after the reaction takes place.

In this work, we first thoroughly characterise the effect of handhold complementarity in the displacement rate. We then demonstrate that the displacement rate can be increased by several orders of magnitude when using $I$-strands with handhold complementarity (Ihc) over identical strands but without handhold complementarity $\left(I_{w c}\right)$. Next, we show that it is possible to both increase the displacement rate and allow the $I T$ product to detach from $N$ when using handholds of moderate length. Finally, we demonstrate the templating of specific, far-from-equilibrium IT complexes via transient handhold interactions. The process we demonstrate - illustrated schematically in Fig.1-d - is a simplified non-catalytic version of far-from-equilibrium templating which, nonetheless, retains the central challenge of producing a non-equilibrium distribution of products through transient recognition interactions with a template.

\section{Results and discussion}

Handhold-mediated strand displacement. The proposed handhold mechanism is as follows (Fig.2-c): First, $I_{h c}$ recognises and reversibly binds to the handhold, producing a transient 3 -stranded complex that colocalises $I_{h c}$ and the duplex $T N$; then $I_{h c}$ displaces $N$ from $T$. A short proximal toehold between $I_{h c}$ and $T$ can further ac- celerate the displacement step. The distinguishing feature of handholds relative to toeholds is that $I$ recognises $N$ but uses this recognition to strongly bind to $T$ while, in the process, weakening the interaction between $N$ and $T$. It is this reversal of the reaction topology relative to TMSD that inspires the name HMSD. The crucial functional difference between TMSD and HMSD is that the formation of the $I T$ product does not produce binding cooperativity between the handhold recognition interaction (between $N$ and $I$ ) and the displacement domain binding (between $I$ and $T$ ). Hence, the product IT can spontaneously detach from $N$ 's handhold, breaking the recognition interaction.

We therefore hypothesize that handholds can provide reaction specificity by increasing the displacement rate of a particular $I$. But, in contrast to toeholds, for short enough handholds, the handhold detachment reaction can dominate the reverse re-binding reaction, resulting in the production of IT complexes free in solution, instead of bound to $N$. Since the detached $I T$ product resultant from just HMSD have similar stability as $T N$, the displacement process can be driven by introducing a small proximal toehold, mismatches in $N,{ }^{32}$ or coupling it with a downstream displacement reaction that acts as a product sink. 
a)

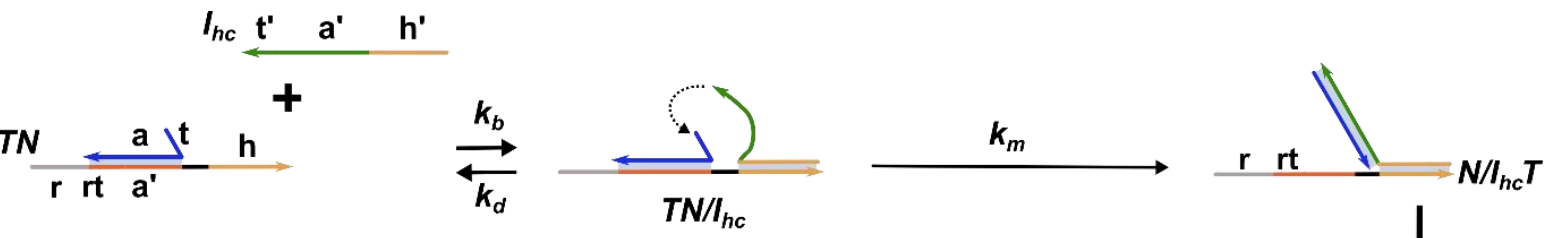

With handhold complementarity (h')

Without handhold complementarity $\left(x^{\prime}\right)$

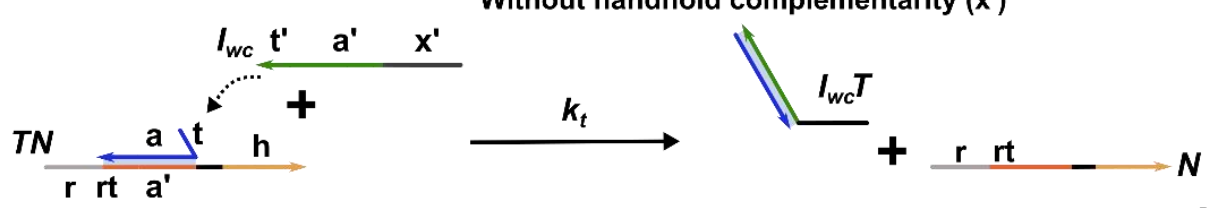

b)
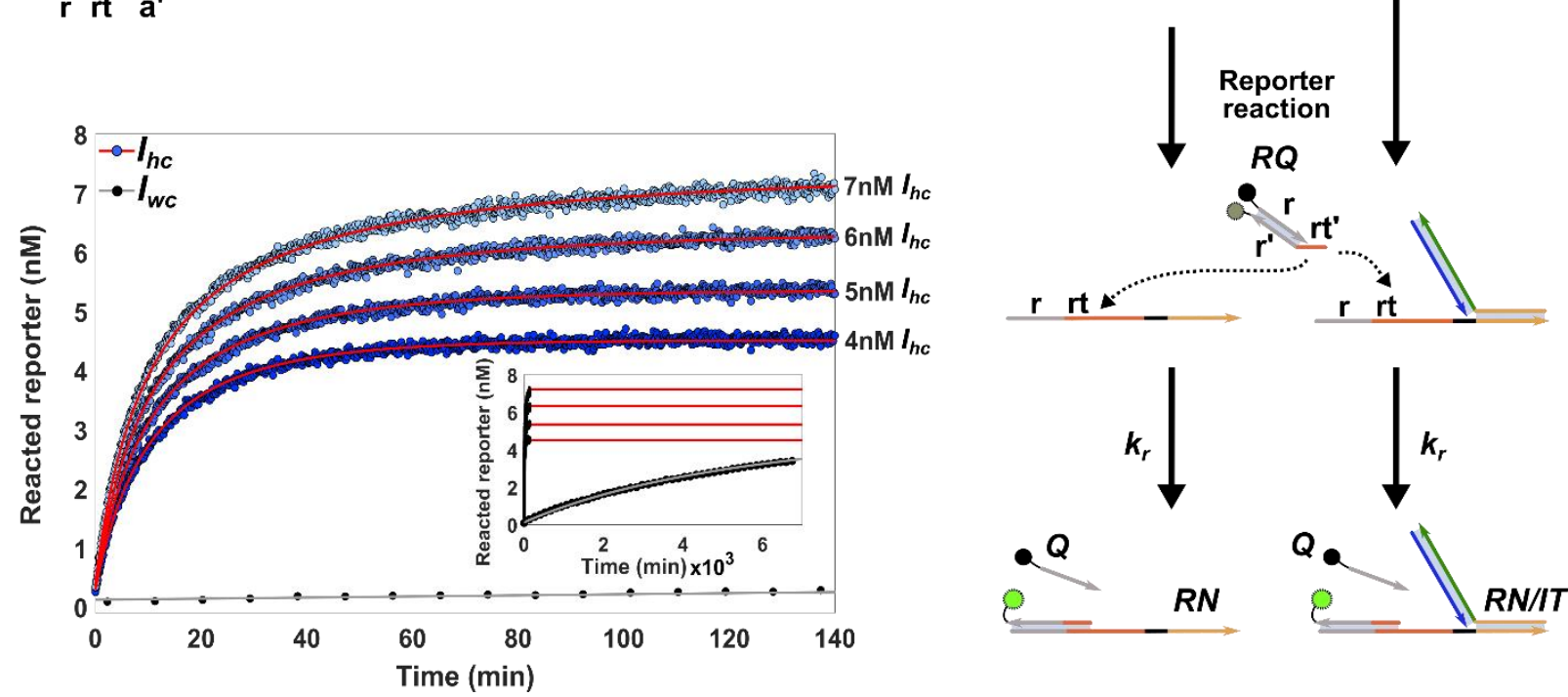

Figure 3: Handhold complementarity substantially enhances displacement rates for short toeholds. a) Handhold-mediated strand displacement (HMSD) characterisation and fluorescent reporter $(\boldsymbol{R} Q)$ reaction: During HMSD characterisation, several target-incumbent duplexes $(T N)$ with different combinations of proximal toehold $(\mathrm{t})$ and handhold $(\mathrm{h})$ lengths are tested. To test the handhold contribution to the kinetics, each $T N$ is triggered with different concentrations of invader strands, with $\left(I_{h c}\right)$ and without $\left(I_{w c}\right)$ handhold complementarity. Completion of the displacement reaction is followed via the external reporter $R Q$. Once $T$ is displaced from $N$, the first 6 nucleotides (nt) of the 5' edge of the displacement domain (rt) can trigger the displacement of $Q$ from the reporter, independently of the handhold occupation, producing a fluorescence signal. b) Measured and fitted fluorescence traces: Representative results for a reaction mediated by a 2 nt t- $T$ strand and $7 \mathrm{nt} \mathrm{h-N}$ strand. A single set of reaction parameters is fitted simultaneously to all the $I_{h c}$ trajectories (red), as outlined in Supplementary Note XVI. Conditions: $[R Q]_{0}=15 \mathrm{nM},[T N]_{0}=10 \mathrm{nM},\left[I_{h c}\right]_{0}=[7-4] \mathrm{nM}, 1 \mathrm{M} \mathrm{NaCl}$ in $1 \mathrm{x}$ TAE at $25^{\circ} \mathrm{C}$. Final fluorescence signal is used to confirm experimentally the precise reagent concentrations as described in Supplementary Note I. Inset: Kinetics for 6nM of $I_{w c}$ (grey), alongside the data from the main figure.

We first test the influence of different handhold and proximal toehold lengths on strand displacement kinetics. We monitor strand displacement with an external fluorescence reporter to minimise fluorophore interference ${ }^{33}$ in the reaction system. The external reporter is a DNA duplex $(R Q)$ labelled with a quenched fluorophore. As shown in Fig.3, $N$ triggers $R Q$ by conventional TMSD, producing fluorescence only after $I T$ formation. The toehold in $R Q$ is complementary to 6 nucleotides (nt) in the 5' edge of $N$ 's displacement domain, and so, reporter triggering does not happen unless $N$ has been displaced. Clamp domains are also added to both sides of $R Q$ to minimise any leak reactions, which are indeed negligible as shown in Supplementary Note VIII. ${ }^{34}$

Typical HMSD reactions consist of adding $I_{h c}$ to a solution containing $T N$ and $R Q$. We also compare HMSD kinetics to those of a TMSD reaction triggered by $I_{w c}$ in the same conditions. We analyse the recorded kinetics by modelling HMSD as a two-step process, where $I_{h c}$ reversibly attaches to the handhold and once bound, triggers the displacement (Fig.2-c):

$$
\begin{aligned}
& k_{b} \quad k_{m} \\
& T N+I \rightleftarrows T N / I \rightarrow N / I T \\
& k_{d}
\end{aligned}
$$

Here, $X / Y$ indicates that species $Y$ is bound to the handhold of $X$. $k_{b}$ and $k_{d}$ are rate constants for $2^{\text {nd }}$ order binding and $1^{\text {st }}$ order detachment of $I_{h c}$ from $N$ 's handhold, respectively. $k_{m}$ is the $1^{\text {st }}$ order rate constant for the strand displacement once $I_{h c}$ is bound to $N$ by its handhold.

Once displacement is completed, the incumbent strand $N$ can trigger the reporter. At this stage, we are agnostic as to whether IT has detached from $N$, assuming that both $N$ and $N / I T$ activate the reporter with the same $2^{\text {nd }}$ order rate constant $k_{r}$.

$$
\begin{gathered}
N / I T+R Q \stackrel{k_{r}}{\rightarrow} R N / I T+Q, \\
N+R Q \stackrel{k_{r}}{\rightarrow} R N+Q .
\end{gathered}
$$

We treat all displacement reactions as effectively irreversible, even in the absence of a proximal toehold to drive the reaction, because $R Q$ acts as a sink for displacement products. 
a)

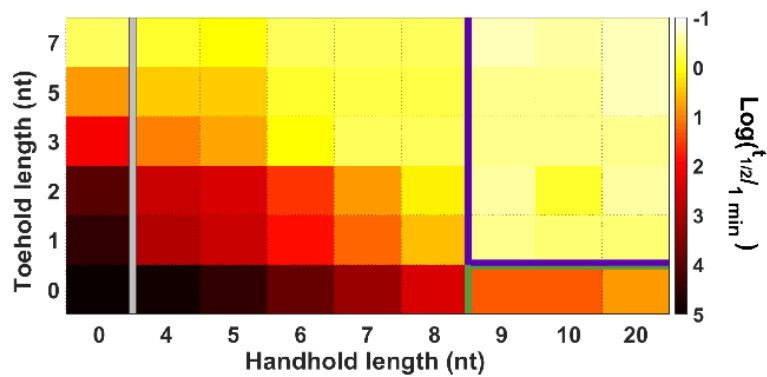

b)

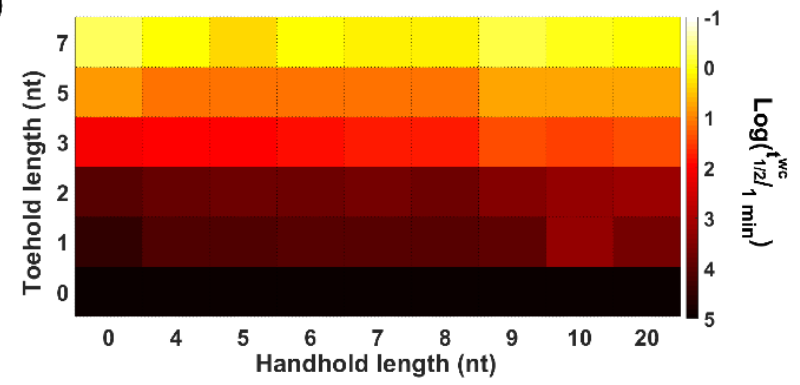

c)

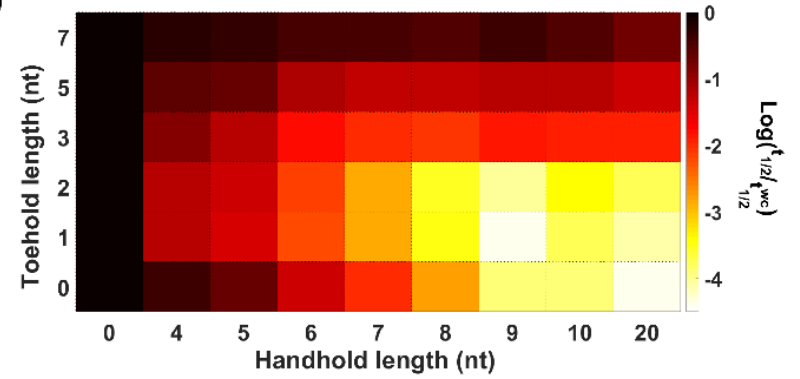

Figure 4: Handhold-complementarity systematically enhances strand displacement rates for short toeholds, as inferred from the fits shown in Supplementary Table 15. a) Half-lives $\left(t_{1 / 2}\right)$ for HMSD. The analysis shows three different reaction regimes: $2^{\text {nd }}$ order reactions with reversible handhold binding (from 4 to $8 \mathrm{nt}$ handhold) and with effectively irreversible handhold binding (outlined in purple); Also a $1^{\text {st }}$ order reaction regime, where the limiting factor is the displacement once bound to the handhold (outlined in green). Reactions in the absence of a handhold are outlined in grey. Displacement is systematically favourable even in the absence of toehold due to the downstream $R Q$ reaction acting as a sink. $t_{1 / 2}$ calculated for $\left[I_{0}=6 \mathrm{nM}\right.$ and $[T N]_{0}=10 \mathrm{nM}$ with parameters inferred at $25^{\circ} \mathrm{C}$. b) Half-lives for $I$ strands without handholdcomplementarity $\left(t_{1 / 2}^{w c}\right)$. Handhold presence in $N$ produces negligible displacement rate changes when $I$-strands lack handhold-complementarity. $t_{1 / 2}^{W C}$ is calculated in the same conditions as $t_{1 / 2}$. c) Comparison between $t_{1 / 2}$ and $t_{1 / 2}^{w c}$. HMSD can reduce displacement $t_{1 / 2}$ up to four orders of magnitude when compared to displacement triggered with $I$-strands without handhold-complementarity. $t_{1 / 2}$ difference translates as an increment of the displacement rate of more than 4 orders of magnitude. The displacement rate increment produced by handhold-complementarity is substantially higher in the regime of toeholds below $3 \mathrm{nt}$.

To complete the model, we also consider displacement of $N$ from $I$ by direct interactions with the toehold, bypassing the handhold. These reactions, with a $2^{\text {nd }}$ order rate $k_{t}$, could occur for both $I_{h c}$ and $I_{w c}$. We estimate the value of $k_{t}$ for every HMSD system from experiments triggered with $I_{w c}$. Toehold-only triggering is essentially a leak reaction, and our results will identify a regime where these leaks are extremely slow compared to the intended handhold-triggered reaction. See Supplementary Note XI for the full implementation of the reaction model as an ODE system.
We numerically solve the ODE system to fit a single set of values for $k_{d}, k_{m}$ and $k_{b}$ to the fluorescent traces produced for each reaction system at a range of $I_{h c}$ concentrations. We obtain the values for $k_{r}$, the constraints for $k_{b}$, and the competing TMSD rate $k_{t}$, from the results of complementary experiments (See Experimental methods). The fitted results (Supplementary Table 15) reveal that HMSD can operate in three different reaction regimes determined by the behaviour of the intermediate species TN/I. The three defined reaction regimes are $1^{\text {st }}$ order and $2^{\text {nd }}$ order with either reversible or effectively irreversible handhold-binding. For the tested HMSD systems, the most frequent reaction regime is the $2^{\text {nd }}$ order rate with reversible handhold-binding (Condition: $k_{m}, k_{b}\left[I_{0}<k_{d}\right.$ ,with $\left[I_{0}\right.$ as the initial invader concentration). In this reaction regime, the complex $T N / I$ is short-lived, reaching a quasi-steady state concentration of $[T N / I]=[I][T N] k_{b} /\left(k_{m}+k_{d}\right)$ that balances detachment and migration events with binding. Branch migration events occur from this state at a rate $k_{b}$, and so combining the overall HMSD rate with the direct TMSD rate yields an apparent $2^{\text {nd }}$ order displacement rate:

$$
k=k_{b} \frac{k_{m}}{k_{m}+k_{d}}+k_{t} .
$$

When handholds become longer than $8 \mathrm{nt}\left(\Delta \mathrm{G}^{\circ}\right.$ binding $<-12.39$ $\mathrm{Kcal} / \mathrm{mol})^{35}$ we observe effectively irreversible handhold binding. For systems with a non-zero proximal toehold the kinetics are of $2^{\text {nd }}$ order. In this regime, $T N / I$ is quickly formed and consumed by the branch migration reaction. The above analysis holds, but with $k_{m} \rightarrow \infty$ : all handhold-binding events are stable on the timescale of branch migration, which is fast relative to binding. (Condition: $k_{d}$, $k_{b}\left[I_{0}<k_{m}\right)$. The resultant $k$ is insensitive to changes in $k_{m}$ and $k_{d}$ $\left(k \approx k_{b}+k_{t} \approx 10^{6} \mathrm{M}^{-1} \mathrm{~s}^{-1}\right)$. The $1^{\text {st }}$ order reaction regime occurs in systems with handhold binding irreversibility and no proximal toehold (Condition: $k_{b}[I]_{0}>k_{d}, k_{m} ; k_{t} \approx 0$ ). In this regime, $T N / I$ is quickly and irreversibly formed, accumulating due to the handhold-binding stability and the reduced branch migration rate. The intermediate thus reaches a concentration of $[T N / I]=\min \left([T N]_{0},\left[I_{0}\right)\right.$, and then undergoes a first order transition to the displaced state with rate constant $k_{m}$ (Mean fitted $k_{m}$ at $25^{\circ} \mathrm{C}=1.21 \pm 0.29 .10^{-3} \mathrm{~s}^{-1}$, see Supplementary Tables 15 and 16).

Given the existence of reaction regimes with $1^{\text {st }}$ and $2^{\text {nd }}$ order rates, we cannot use each HMSD system's apparent rate constant for direct comparison of the different systems tested. Instead, we use the fitted rate constants to infer each HMSD system half-life $\left(t_{1 / 2}\right)$ at a default reference concentration, as described in the Experimental methods, to use it as a comparable measure of each system reaction speed (Fig.4-a). From these results, we prove that handhold complementarity decreases $t_{1 / 2}$ by several orders of magnitude, analogously to toeholds in conventional TMSD. For HMSD systems with a $1 \mathrm{nt}$-long toehold and saturating handhold lengths (>8 nt), the obtained $t_{1 / 2}$ is consistent with conventional TMSD at saturating toehold lengths. In Fig.4-b, we report the half-life for systems triggered with $I_{w c}\left(t_{1 / 2}^{w c}\right)$. As expected, these systems only react by conventional TMSD, with rate $k_{t}$ and $t_{1 / 2}^{w c}$ is insensitive to handhold presence. Hence, we can conclude that $t_{1 / 2}^{w c}$ is mainly determined by proximal toehold length.

To estimate the contribution of handholds to the kinetics, we plot the ratio $t_{1 / 2} / t_{l / 2}^{w c}$ for every system in Fig.4-c. The handhold contribution to the kinetics is especially notable for systems with proximal toeholds smaller than $3 \mathrm{nt}$ and handholds longer than $6 \mathrm{nt}$, where handhold-complementarity can reduce $t_{1 / 2}$ by four orders of magnitude. This reduction of $t_{1 / 2}$ in our experimental conditions translates to $k$ increments of more than 4 orders of magnitude (Supplementary Figure 20). 
a)
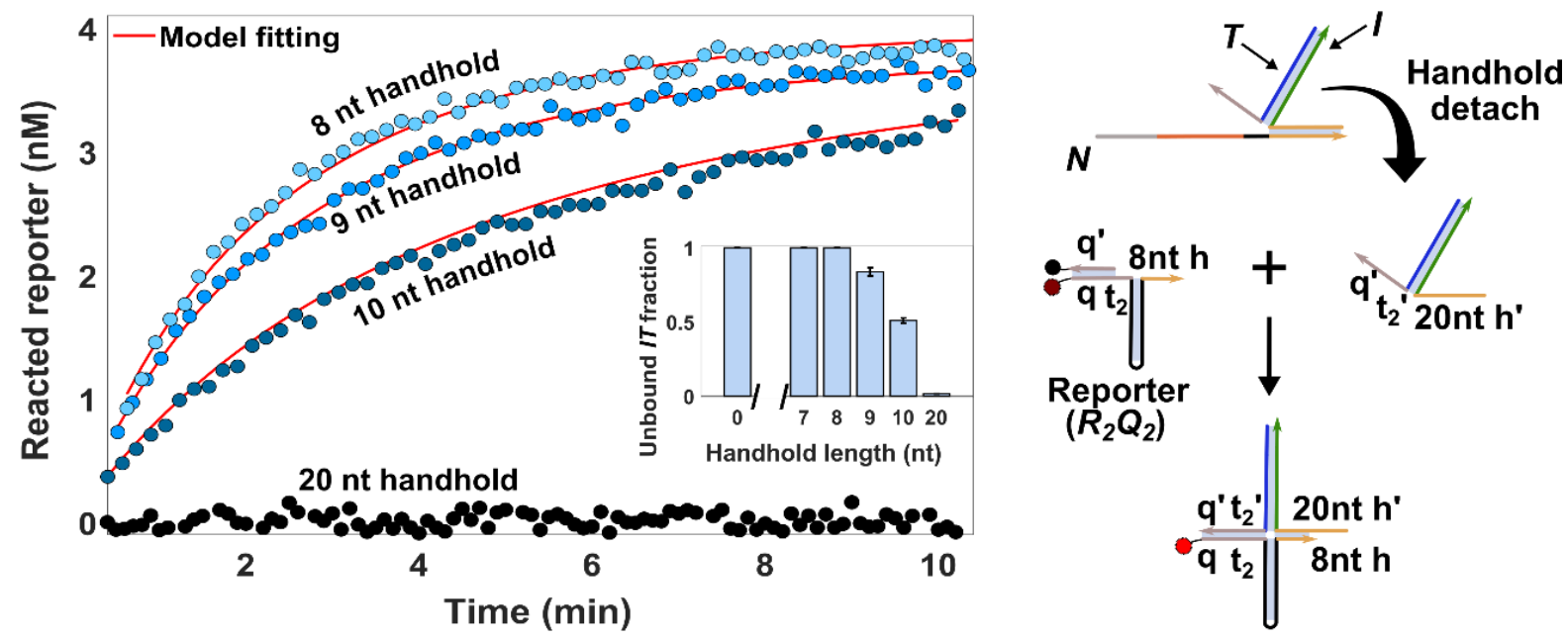

b)

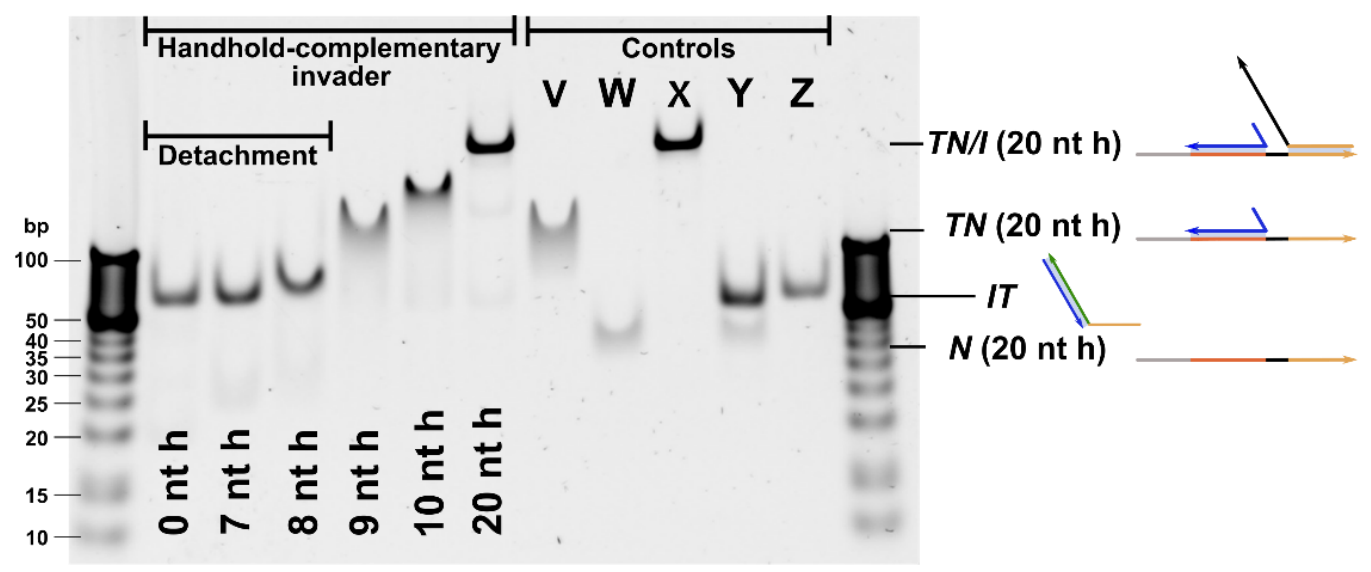

Figure 5: Handholds of moderate length accelerate displacement while allowing for detachment. a) Kinetic analysis. HMSD triggered under the same experimental conditions as described in Fig. 3 with $\left[I_{0}=4 \mathrm{nM}\right.$ and a $3 \mathrm{nt}$ proximal toehold. Once HMSD reaches steady state, reporter $R_{2} Q_{2}$ is added to react with any detached $I T$ by an associative toehold strand displacement. The rate of triggering is negatively correlated with handhold length, saturating for handholds shorter than $9 \mathrm{nt}$. Inset: The unbound fraction of IT after HMSD is complete is inferred from fitting $K_{e q}$ to $R_{2} Q_{2}$ kinetics as outlined in Supplementary Note XVII. b) Native polyacrylamide gel electrophoresis. 12.5 mM $\mathrm{MgCl}_{2}$ in $0.5 \mathrm{x} \mathrm{TBE}$ at $25^{\circ} \mathrm{C}$. A qualitative confirmation that, for handholds up to $8 \mathrm{nt}$, IT is mostly detached from the handhold at equilibrium. V: $T N$ duplex. W: $N$-strand. X: $T N$ duplex + random strand with handhold-complementarity (results in a 3-strand complex since it cannot displace $N$ ). Y: $T N+I$ without handhold-complementarity (results in $I T+N$ ). Z: $I T$ duplex. All controls have a handhold length of 20 nt.

Product Separation. For HMSD to underlie far-from-equilibrium templating, handhold binding must be transient and release the majority of $I T$ after the displacement. To verify release, we design a second reporter DNA duplex $\left(R_{2} Q_{2}\right)$ to detect detachment of $I T$ from $N$. Like $R Q$, we label $R_{2} Q_{2}$ strands with a quenched fluorophore. $R_{2} Q_{2}$ has an associative toehold ${ }^{23}$ formed by two singlestranded domains brought into proximity by a hairpin (Fig.5-a).

As shown in Supplementary Figure $14, R_{2} Q_{2}$ only reacts at highspeed with $I T$ duplexes free in solution. The reaction that describes the kinetics of $R_{2} Q_{2}$ is:

$$
I T+R_{2} Q_{2} \stackrel{k_{r 2}}{\rightarrow} R_{2} / I T+Q_{2},
$$

where $k_{r 2}$ is a $2^{\text {nd }}$ order rate constant. We infer $k_{r 2}$ value from systems with no handhold, where $I T$ is completely detached $\left([I T]=\left[I_{0}\right.\right.$; $[N / I T]=0)$. For systems with a handhold, it is possible to extrapolate the amount of detached $I T$ from the kinetics resultant from adding $R_{2} Q_{2}$ after HMSD reaches completion. We estimate the equilibrium constant:

$$
K_{e q}=\frac{[N][I T]}{[N / I T]},
$$

assuming that $I T$ remains in instantaneous equilibrium with the $N / I T$ complex, which is reasonable given that $k_{b}>k_{r 2}$. Hence, we fit the value of $K_{e q}$ with the experimental $R_{2} Q_{2}$ traces as described in the Experimental methods section. The fitted values for $K_{e q}$ reveal that, when HMSD reactions have reached completion, $>99 \%$ of the produced $I T$ is free in solution for handholds shorter than $9 \mathrm{nt}$ (See Experimental methods, Fig.5-a). For handhold lengths of 9 and 10 nt, the three-stranded complex N/IT becomes more prevalent, as evidenced by the slowdown in the reporter kinetics. For handholds $20 \mathrm{nt}$ long, all IT is sequestered in the N/IT form and reporter triggering is minimal.

We further perform a qualitative confirmation of HMSD products detachment with PAGE. ${ }^{36}$ To prevent sample heating, the gel is not resolved using identical buffer conditions: buffer $\mathrm{NaCl}$ content is exchanged for a lower concentration of $\mathrm{MgCl}$. Although the stability of the complexes could slightly vary between buffer conditions, the gel results confirm the substantial presence of threestranded N/IT complexes only for handholds longer than $8 \mathrm{nt}$ (Fig.4-b). Furthermore, we show in Supplementary Figure 5 that the PAGE buffer does not qualitatively alter the detachment observed with $R_{2} Q_{2}$. 
a)

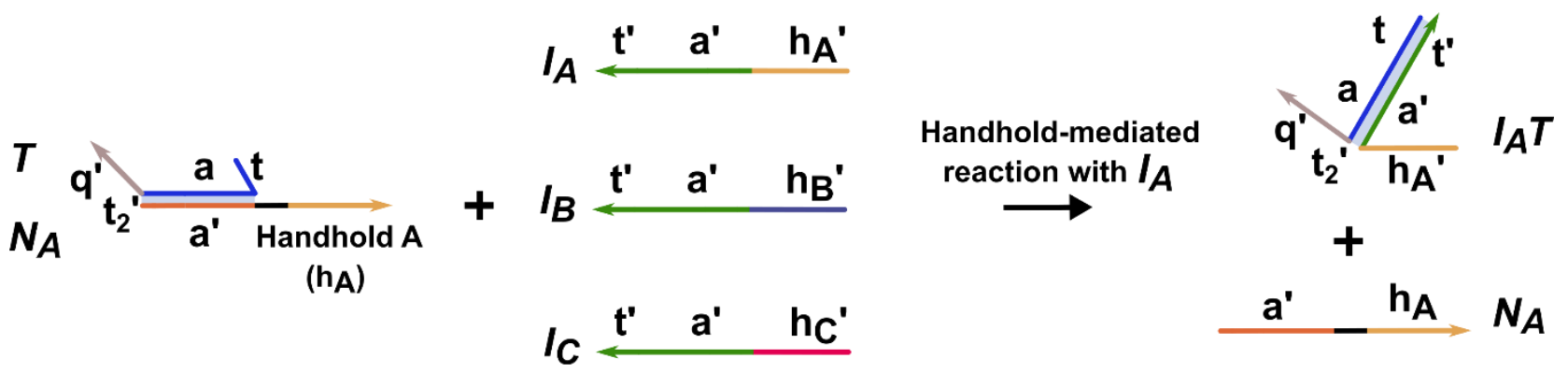

b)

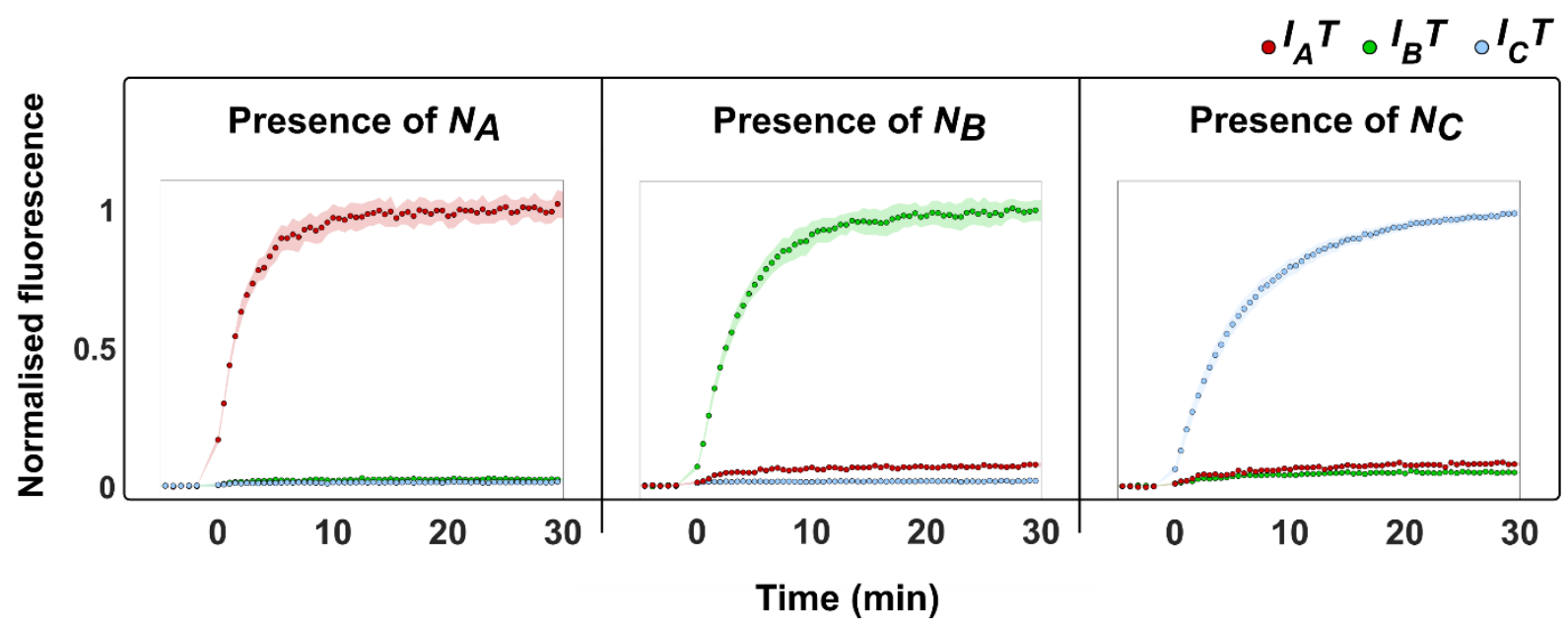

Figure 6: Selective production of a single complex from approximately equally stable alternatives $I_{A} T, I_{B} T$, and $I_{C} T$ through handhold-mediated templating. a) The specific production of $I_{x} T$ complexes is determined by the handhold sequence of three different strands $N_{x}$. Complementarity between $I_{x}$ and $T$ is identical for all the possible $I_{x} T$ products. Therefore, product selectivity cannot rely on a bias in equilibrium. The transient interaction with a complementary handhold (h) determines the rate of the reaction, without altering the relative product stability, and thereby pushing product distributions out of equilibrium. b) Three reporters are used to detect the formation of free $I_{x} T$ complexes via HMSD ( $8 \mathrm{nt}$ handhold/2 $\mathrm{nt}$ proximal toehold). Sequence differences in $\mathrm{h}$ result in slightly different trajectories, but the unambiguous ability to select any product is confirmation of the success of non-equilibrium templating. The shaded area represents the standard error of the mean fluorescence for each experiment $(\mathrm{n}=4)$. Conditions: $\left[T N_{x}\right]=10 \mathrm{nM},\left[I_{x}\right]=25 \mathrm{nM}$ for all three $I_{x}$ strands, and 30 $\mathrm{nM}$ of each specific reporter, $1 \mathrm{M} \mathrm{NaCl}$ in $1 \mathrm{x} \mathrm{TAE}$ at $25^{\circ} \mathrm{C}$.

Templating non-equilibrium assembly. We next harness HMSD to produce a far-from-equilibrium ensemble of $I T$ products through templating. We perform an iteration of the experiment depicted in Fig.5-a, but with three competing invading strands $I_{A}, I_{B}$ and $I_{C}$ (Fig.6-a). Each invader $I_{A}, I_{B}$ and $I_{C}$ is equally complementary to a $T$ strand and its 2 nt toehold, and complementary to one of the different $8 \mathrm{nt}$ handholds located in templates $N_{A}, N_{B}$ and $N_{C}$.

From the law of mass action, the equilibrium yields of free $I T$ products would be related by expressions of the form:

$$
\frac{\left[I_{A} T\right] /\left[I_{A}\right]}{\left[I_{B} T\right] /\left[I_{B}\right]}=\exp \left(\left(G_{B}-G_{A}\right) / \mathrm{RT}\right)
$$

in which $G_{i}$ is the standard free energy of formation of $I_{i} T$. These ratios are independent of which template $N$ is present, and predict relatively similar yields of all products, since the free energies of formation of all $I T$ are similar. Indeed for an equimolar mixture of all the strands in our experimental conditions, NUPACK ${ }^{37}$ predicts a ratio of product formation of $0.21 / 0.27 / 0.52$ for the products $I_{A} T$, $I_{B} T$ and $I_{C} T$, respectively, equivalent to a free-energy difference of only around 1RT between the products.

However, we use handhold complementarity with either $N_{A}, N_{B}$ or $N_{C}$ to produce product ensembles that are heavily biased towards either $I_{A} T, I_{B} T$ or $I_{C} T$, respectively - as illustrated schematically in Fig.1-d. To do so, we mix all three invaders with initial solutions containing one species of the duplexes $N_{A} T, N_{B} T$ or $N_{C} T$. Since handholds of this length produce only transient binding, the products $I_{A} T, I_{B} T$ and $I_{C} T$ will overwhelmingly dissociate from $N_{A}, N_{B}$ and $N_{C}$ respectively.

We, add three different reporters, analogous to $R_{2} Q_{2}$, that detect the possible products $I_{A} T, I_{B} T$ and $I_{C} T$, when free in solution. Although the presence of $N_{X}$ cannot provide an equilibrium bias towards a detectable complementary $I_{x} T$ product free in solution, we nonetheless observe in Fig.6-b, the selective formation of any single $I_{x} T$ product, free in solution, can be templated by the appropriate transient handhold interaction from a solution containing all three invaders. HMSD is therefore capable of templating the system intentionally into specific kinetic traps for the formation of farfrom-equilibrium product ensembles.

\section{Conclusion}

We have introduced HMSD not only as a simple and rational method to control strand displacement kinetics, but to add an important feature to DNA nanotechnology: templating of far-fromequilibrium assemblies. Other superficially similar processes ${ }^{23-25}$, 38 do not involve a transient recognition interaction; when using toeholds as the source of specificity, they are permanently sequestered in the product complex due to cooperativity with the remainder of the base pairs in the product. In HMSD, this cooperativity does not arise; the base pairs between $N / I$ and $N T$ are essentially independent in the product state. 
In the current article, we have used transient recognition interactions with a template to selectively assemble a single DNA duplex from an ensemble of equally stable alternatives. The product state is far from the equilibrium distribution of approximately equal concentrations of all duplexes; HMSD thus successfully channels the system towards this non-equilibrium state of high free energy. We have also characterised the kinetics of HMSD and introduced a simple model to predict it. We hope our work will establish HMSD as an orthogonal reaction in DNA nanotechnology that can be used to create non-equilibrium assemblies for a great variety of uses.

The production of non-equilibrium assemblies is essential to biological processes such as transcription and translation. The second crucial requirement of these processes in nature is that the templates act catalytically - a single template molecule can be used to produce multiple products, whose assembly is kinetically suppressed in the absence of the template. The next challenge is to incorporate HMSD into a genuinely catalytic mechanism for dimerization (Fig.1-c), rather than initiating the templates with the $T$ monomer pre-loaded as in this work (Fig.1-d). A pool of functionalised strands in solution could then be continuously templated, working as a synthetic analogue of aminoacyl-tRNA synthetases, the molecules responsible for selectively combining tRNAs with their cognate amino acids. ${ }^{39}$

Eventually, HMSD has the potential to underlie the catalytic production of sequence-specific polymers from longer templates, to create synthetic analogues of transcription and translation. Although many challenges remain, HMSD solves an important problem in this context. A central challenge of engineering such systems in a purely chemically-driven context has always been "product inhibition": the cooperativity of interactions between long products and templates tends to prevent detachment unless the complexes are disrupted by another source of energy. ${ }^{14-16,25,29,31}$ HMSD is explicitly designed to overcome product inhibition; much of the free energy released by the binding between $I$ and $T$ is diverted into weakening the binding between $T$ and the template $N$, so that the $I T$ complex does not bind more strongly to the template than $I$ alone. This mechanism - using the free energy of a reaction to enhance dissociation from a template - was recently postulated to be ideal in a theoretical analysis of minimal catalysts. ${ }^{40}$

Other systems have been proposed in which the dimerization process weakens the interaction of the product with the template, 14-16 but a particular advantage of HMSD is that the binding of $I$ to $N$ is not weakened by the branch migration process, only the binding of $T$ to $N$. Thus, it is possible to imagine a long template with multiple handhold sites; an invader $I$ can bind to a target $T$ via HMSD, then, remain bound by a long handhold and in turn becoming the target for the next displacement. This way, creating a templated polymer of DNA strands step by step. A shorter handhold for the final step would allow full detachment of the complete copy at the appropriate time.

\section{Experimental methods}

DNA sequence design. DNA sequences that minimise undesired interactions during HMSD were designed with bespoke scripts using the NUPACK server (http://www.nupack.org). ${ }^{37}$ All strands were purchased from Integrated DNA Technologies (IDT) with HPLC purification and normalized at $100 \mu \mathrm{M}$ in LabReady ${ }^{\circledR}$ buffer. All used sequences are listed by function (Incumbent $(N)$, Target $(T)$, Invader $(I)$ and reporters) are collected in Supplementary Tables 20 to 23 .

DNA duplex preparation. $T N$ duplexes were formed by combining $200 \mathrm{nM}$ of $T$ with a $10 \%$ excess of $N$ to ensure the anneal of every $T$. For Reporter characterisations, the $T N$ anneal was prepared with a $10 \%$ excess of $T$ since $T$ is inert during those assays. Annealing was performed in the experimental buffer (TAE $1 \mathrm{X}$ and $1 \mathrm{M} \mathrm{NaCl}, \mathrm{pH} 8.3$ ), except for PAGE samples which were annealed in a solution of TAE $1 \mathrm{X}$ and $12.5 \mathrm{mM} \mathrm{MgCl} 2$. Strands forming reporter complexes, except $R_{2} Q_{2}$, were mixed at a concentration of $300 \mathrm{nM}$. $R Q$ was annealed in experimental buffer with a $10 \%$ excess of the quencher-labelled strand. $\operatorname{Rep}_{A}, \operatorname{Rep}_{B}$ and $\operatorname{Rep}_{C}$ were annealed with a $60 \%$ excess of the quencher-labelled strand to minimise the crosstalk between reporter complexes. Each $100 \mu \mathrm{L}$ of $T N$ or reporter solution, except $R_{2} Q_{2}$, was annealed by heating to $95^{\circ} \mathrm{C}$ for $4 \mathrm{~min}$ and cooling to $20^{\circ} \mathrm{C}$ at a rate of $1^{\circ} \mathrm{C} / \mathrm{min}$. $R_{2} Q_{2}$ reporter was annealed at a concentration of $60 \mathrm{nM}$ with a $10 \%$ excess of the quencher-labelled strand. Each $500 \mu \mathrm{L}$ of $R_{2} Q_{2}$ was heated up to $95^{\circ} \mathrm{C}$ for 5 minutes and then cooled to room temperature over 1 hour.

Bulk fluorescence spectroscopy. Bulk fluorescence assays were carried out in a Clariostar Microplate reader (BMG LABTECH) using flat $\mu$ Clear bottom 96-well plates (Greiner) and reading from the bottom. Kinetics were recorded, unless stated otherwise, after injecting $50 \mu \mathrm{L}$ of the reaction trigger in $150 \mu \mathrm{L}$ of experimental buffer containing the reactant species and the reporter complex (pump speed: $430 \mu \mathrm{L} / \mathrm{s}$ ). The final mixture was shaken for 3 seconds (double-orbital, at $400 \mathrm{rpm}$ ). Injected and reacting volumes were previously preheated to the experiment temperature. The samples were contained in Eppendorf ${ }^{\circledR}$ Lobind tubes, and the plate reader's injector system passivated by incubating with BSA $5 \%$ during 30 minutes to maximise concentration reproducibility during the assays ${ }^{41}$.

$R Q$ reporter was labelled with $\mathrm{Cy} 3$ and $\mathrm{FQ}$ IowaBlack ${ }^{\mathrm{TM}}$ quencher (Excitation: $530 / 20 \mathrm{~nm}$; emission: $580 / 30 \mathrm{~nm}$ ). $R_{2} Q_{2}$ reporter was labelled with Cy5 and RQ IowaBlack ${ }^{\mathrm{TM}}$ quencher (Excitation: 610/30 nm; emission: 675/50 nm). Signal was averaged for 20 flashes per data point. Experiments with durations over 12 hours were averaged for 100 flashes in a spiral area scan per data point. Reporter characterisation assays were measured individually with 1 flash/datapoint due to the fast rate of the reaction. Every system was assayed with at least three different reaction trigger concentrations.

Fluorescence calibrations. Fluorescence calibrations were made for the reporter DNA duplexes used during characterisation assays ( $R Q / \mathrm{Cy} 3$ and $\left.R_{2} Q_{2} / \mathrm{Cy} 5\right)$. Calibrations aimed to estimate the units of fluorescence produced per $\mathrm{nM}$ of the fluorophore-labelled strand to quantify the amount of reacted reporter during the assays. Calibration curves ranged from 1 to $15 \mathrm{nM}$ in $200 \mu \mathrm{L}$ and were made by triplicate from stock solutions at $100 \mu \mathrm{M}$. One replica included both fluorophores (Cy3 and $\mathrm{Cy} 5)$ to rule out bleed-through fluorescence. The calibration protocol and results are collected in Supplementary Notes IV and V and Supplementary Figures 7 to 10.

General protocol for characterisation experiments. HMSD systems with a combination of 9 different handhold lengths $(0,4,5,6,7,8,9,10$ and $20 \mathrm{nt})$ and 6 different proximal toeholds $(0,1,2,3,5$ and $7 \mathrm{nt})$ were tested at $25^{\circ} \mathrm{C}$. A smaller set of systems were also characterised at $37^{\circ} \mathrm{C}$ (Supplementary Table 17). The effect of a spacer sequence located after the handhold of $N$ was also tested. Spacer lengths of $0,1,2,3,5$ and $8 \mathrm{nt}$ were included for systems with handhold lengths of 0,5 and $20 \mathrm{nt}$ and proximal toehold lengths of 0,2 and $7 \mathrm{nt}$. The addition of a spacer sequence did not have a clear effect on HMSD except for systems with $5 \mathrm{nt}$ handholds and proximal toeholds of 0 or $2 \mathrm{nt}$, where a spacer length of $2 \mathrm{nt}$ was found optimal (Supplementary Table 16). Based on this finding, all systems reported in the main text contain a 2 nt spacer in $N$.

Characterisation protocols were adapted from the method employed by Srinivas et al. ${ }^{42}$ Each experiment consisted of the system kinetics and a set of complementary measurements. These complementary measurements quantified the fluorescence baseline and estimated the concentration of each species in the system from the fluorescence signal measured after sequentially triggering the reaction of all the species (Supplementary Figures 1 and 2). Intended species concentrations during the experiments, unless specified otherwise, were $[R Q]_{0}=15 \mathrm{nM},[T N]_{0}=10 \mathrm{nM}$ and $\left[I_{0}=[4-8] \mathrm{nM}\right.$, at 
a reference reaction volume of $200 \mu \mathrm{L}$. The excess of $[R Q]_{0}$ and $[T N]_{0}$ over $[I]_{0}$ was used to ensure that all invader strands reacted; this allowed us to estimate reagents' concentrations from fluorescence signals.

The purpose of considering a range of concentrations for the $I$ strand was to test the robustness of the reaction kinetics for their $2^{\text {nd }}$ order and pseudo- $1^{\text {st }}$ order approximations. Simultaneously, the experimental replicas provided an estimate of the statistical error in the inferred reaction kinetics. Supplementary Notes I to III contain a detailed description of the quantification method and the measurements during each type of characterisation assay.

General fitting procedure. All fittings were performed with MATLAB ${ }^{\circledR}$ R2019a Optimization Toolbox. Besides the relevant rate constants, each fitting also allowed a variation of the fitted curve's reaction initiation time $(t o)$. This correction amended the initial leak of fluorescence signal produced by defective duplexes that can trigger instantaneously the reporter $R Q$. to fitting became especially relevant for slow reactions where the initial increment of fluorescence could be equal to that after several hours of reaction. Also, for reactions that consumed at least $90 \%$ of its reaction trigger, the estimated trigger concentration was fitted to correct any discrepancy with the fluorescence signal of the kinetics equilibrium plateau. Supplementary Notes XI to XVII contain a detailed description of the fitting procedures.

Reporter characterisation. The value of $k_{r}$ was obtained experimentally for every species of $N$. Experiments consisted of a solution of $R Q$ triggered by $N / I T\left([N / I T]_{0}=[4-8] \mathrm{nM}\right)$. The injected $N / I T$ solution was obtained by mixing $T N$ (toehold $7 \mathrm{nt}$ long) with a $20 \%$ excess of $I_{h c}$. Each individual fluorescence trace was used to fit the analytical solution of the ODE describing equation 2 :

$$
[R N]=[N / I T]_{0} \frac{\left(1-\mathrm{e}^{\left(k_{r}\left(t-t_{0}\right)\left([N / I T]_{0}-[R Q]_{0}\right)\right)}\right.}{1-\frac{[N / I T]_{0}}{[R Q]_{0}} \mathrm{e}^{\left(k_{r}\left(t-t_{0}\right)\left([N / I T]_{0}-[R Q]_{0}\right)\right)}},(8)
$$

with $R N$ being the fluorescent species. Modifications in the size and sequence of the $N$ strands produced moderate variation of the fitted $k_{r}$ value. $k_{r}$ variation was particularly relevant during the fitting of HMSD systems with high reaction rates. To minimise the fitting error, subsequent fits used the $k_{r}$ value obtained for the $N$ present in each fitted system. All fitted $k_{r}$ are contained in Supplementary Tables 9 and 10.

Toehold-mediated reaction characterisation. The experimental value of $k_{t}$ was obtained for most of the combinations of handhold and toehold lengths assayed. Otherwise, $k_{t}$ was inferred from the mean of the $k_{t}$ obtained for systems with the same toehold length. Experiments consisted of a solution of $R Q$ and $T N$ triggered by $I_{w c}$. Each individual fluorescence trace was used to fit a reduced ODE model describing equation 4 and a TMSD $2^{\text {nd }}$ order reaction:

$$
T N+I \stackrel{k_{t}}{\rightarrow} N+I T .
$$

Subsequent fits used the $k_{t}$ obtained for each system to account for any unpredicted effect of the handhold length on $k_{t}$. All fitted $k_{t}$ values are collected in Supplementary Tables 15 to 17.

HMSD rate characterisations. A solution of $R Q$ and $T N$ was triggered by $I_{h c}$. For each system, their traces were used simultaneously to fit the ODE model implied by equations 1, 2, 9 and the additional handhold binding/unbinding reactions:

$k_{b}$

$$
\begin{aligned}
& T N+I T \rightleftharpoons T N / I T, \\
& k_{d} \\
& k_{b} \\
& \begin{array}{c}
R N+I \underset{k_{d}}{\rightleftharpoons} R N / I, \\
k_{b}
\end{array}
\end{aligned}
$$

$$
\begin{gathered}
k_{b} \\
R N+I T \underset{\rightleftharpoons}{\rightleftharpoons} R N / I T . \text { (12) } \\
k_{d}
\end{gathered}
$$

The species TN/IT resultant from equation 10 is unable to undergo displacement via the handhold due to the bound IT complex. At the same time, invasion of the free proximal toehold in the $T N$ duplex still occurs with the rate $k_{t}$ :

$$
\stackrel{k_{t}}{T N / I T+I \rightarrow I T+N / I T \text {. (13) }}
$$

The complete mass-action-based ODE model (equations 1, 2, 9, $10,11,12$ and 13) is described in Supplementary Note XI. During the fitting, parameters $k_{r}$ and $k_{t}$ were fixed at the values obtained as described in Reporter characterisation and Toehold-mediated reaction characterisation, respectively. $k_{b}$ was constrained to a certain range, determined by fittings done in systems confidently located in the $2^{\text {nd }}$ order regime with irreversible handhold binding (handhold=20 nt; toehold $>1 \mathrm{nt}$ ).

For the proposed reaction mechanism, most systems operated in the $2^{\text {nd }}$ order rate reaction regime with reversible handhold-binding, where the reaction rate was defined by relative values of $k_{b}, k_{d}$ and $k_{m}$ (equation 4). This fact precluded the fitting algorithm from obtaining comparable absolute values for the rate constants across all tested systems. Constraining $k_{b}$ to a fixed range eased this problem while still producing low error fittings for all tested systems; however, compensatory errors in the ratio $\frac{k_{m}}{k_{m}+k_{d}}$ could mitigate for an inaccurate $k_{b}$. Thus, the effective bimolecular rate constant (equation 4 ) is considered the most reliable descriptor of system kinetics in the $2^{\text {nd }}$ order rate reaction regime. For the $1^{\text {st }}$ order rate reaction regime, the rate is described with $k_{m}$. For all tested systems, the mean values of its fitted parameters and their effective bimolecular rates are collected in Supplementary Tables 15 to 17.

HMSD half-life $\left(\boldsymbol{t}_{1 / 2}\right)$ calculation. Using the rate constants obtained from HMSD rate characterisations, new trajectories were generated for each system with the complete ODE reaction model (Supplementary Note XI). Trajectories were generated for initial conditions $\left[I_{0}=6 \mathrm{nM},[T N]_{0}=10 \mathrm{nM}\right.$ and $[R Q]_{0}=1 \mu \mathrm{M}$. The $t_{1 / 2}$ of a system was defined as the time at which $3 \mathrm{nM}$ of $[I]$ had reacted. The high concentration of simulated $[R Q]_{0}$ made the reporter's reaction effectively instantaneous, removing variability produced by $k_{r}$. Obviating the reporter reaction also allowed calculation of the analytical expression for $t_{1 / 2}^{w c}$ as:

$$
t_{1 / 2}^{w c}=\frac{\ln \left(2-\frac{[I]_{0}}{[T N]_{0}}\right)}{k_{t}\left([T N]_{0}-[I]_{0}\right)}
$$

All calculated $t_{1 / 2}$ and $t_{1 / 2}^{w c}$ are collected in Supplementary Tables 15 to 17.

Detachment assays. $I_{h c}$ was added to a solution containing $R Q$ and $T N$ to produce a final mixture of $I T$ and $N / I T$ $\left([I T]+[N / I T]=[I]_{0}=[4-8] \mathrm{nM}\right)$. After the system reached steadystate, $50 \mu \mathrm{L}$ of $R_{2} Q_{2}$ was injected into the experimental solution $\left(\left[R_{2} Q_{2}\right]_{0}=15 \mathrm{nM}\right)$. During these assays, the $T$ strand contained an additional domain in 3' to trigger reporter $R_{2} Q_{2}$. The kinetics of $I T$ triggering $R_{2} Q_{2}$ were measured for 10 minutes. The initial concentrations for all the strands present in the solution $(I, N$ and $T)$ were inferred from the signal of reporter $R Q$. The results of the controls without reporter $R Q$, shown in Supplementary Table 19, confirmed that the presence of $R Q$ did not significantly alter the equilibrium between $I T$ and $N / I T$. For controls in the absence of $R Q$, concentrations were inferred from the signal of reporter $R_{2} Q_{2}$.

To value of $k_{r 2}$ was fitted, analogously to $k_{r}$, by using the traces of a system without handhold with the analytical solution of the ODE describing equation 5 (See Reporter characterisation and 
Supplementary Figure 21). For systems with a handhold, each individual trace was used to fit the value of $K_{e q}$ with the ODE describing equation 5 , and concentrations constrained by equation 6 . The percentage of detached $I T$ was calculated from the fitted $K_{e q}$, for concentrations $[I]_{0} ;[N]_{0}=10 \mathrm{nM}$. All fitted $K_{e q}$ are collected in Supplementary Table 18.

Polyacrylamide gel electrophoresis (PAGE). The gel for detachment analysis (Figure 5-b) was casted with 12\% 37.5:1 acrylamide:bisacrylamide (Millipore ${ }^{\circledR}$ ) and $12.5 \mathrm{mM} \mathrm{MgCl}_{2}$ (effective concentration $12 \mathrm{mM} \mathrm{MgCl} 2$ ) in TBE $0.5 \mathrm{x}$ and used in the same day. Samples were annealed in the presence of $\mathrm{MgCl}_{2}$ and saturated afterwards with $I_{h c}$ while incubating at $25^{\circ} \mathrm{C}$ (See Duplexes annealing). Controls and O'range ruler 5 bp ladder (Thermo Scientific ${ }^{\mathrm{TM}}$ ) were incubated simultaneously. The gel was pre-ran for $10 \mathrm{~min}$ at $120 \mathrm{~V}$. Then, the samples were loaded and ran for 2 hours at the same voltage. $15 \mu \mathrm{L}$ of each sample were mixed with $3 \mu \mathrm{L}$ of orange DNA loading dye (6x) (Thermo Scientific ${ }^{\mathrm{TM}}$ ) and $17 \mu \mathrm{L}$ of the mixture were loaded in each well. The gel ran in a Mini-Protean ${ }^{\circledR}$ electrophoresis chamber (Bio-Rad) keeping the running buffer $(0.5 \times \mathrm{TBE}$ and $12.5 \mathrm{mM} \mathrm{MgCl} 2)$ at $25{ }^{\circ} \mathrm{C}$ using a water bath. Gels were stained for $30 \mathrm{~min}$ with a GelRed® $3 \mathrm{x}$ solution (Biotium.inc) and visualised in a FluorChem ${ }^{\circledR}$ FC2 imager (Alpha Innotech). Excitation: $300 \mathrm{~nm}$. Bandpass filter: 620/40 nm.

Selective production of complexes far-from-equilibrium. The experimental system contained three reporter complexes: RepA (Identical to $R_{2} Q_{2}$ ) and two analogous reporters: Repв and RepC. Each reporter differed in its distal toehold sequence, which enabled them to specifically recognise the handhold of one of the product complexes $I_{A} T, I_{B} T$ or $I_{C} T$ when free in solution (Supplementary Note X). Repв was labelled with Black Hole Quencher(® 2 and Rhodamine $\operatorname{Red}^{\mathrm{TM}}-\mathrm{X}$ (Excitation: 550/20 nm; emission: 605/40nm). RepC was labelled with FQ IowaBlack ${ }^{\mathrm{TM}}$ quencher and Alexa Fluor ${ }^{\circledR} 488$ (Excitation: 488/14 nm; emission: 535/40 nm).

Each experiment consisted of $190 \mu \mathrm{L}$ of experimental buffer at $25^{\circ} \mathrm{C}$, containing the three reporters $\left(\operatorname{Rep}_{\mathrm{A}}, \operatorname{Rep}_{\mathrm{B}}\right.$ and $\left.\mathrm{Rep}_{\mathrm{C}}\right)$ and the three invader species $\left(I_{A}, I_{B}\right.$ and $\left.I_{C}\right)$. The reaction kinetics were triggered by adding $10 \mu \mathrm{L}$ of $T N_{x}$ complex solution with a specific handhold $\left(T N_{A}, T N_{B}\right.$, or $\left.T N_{C}\right)$. Initial concentrations were $25 \mathrm{nM}$ of each species of $I_{x}, 30 \mathrm{nM}$ of each reporter and $10 \mathrm{nM}$ of $T N_{x}$ complex. An excess of $\left[I_{x}\right]_{0}$ over $\left[T N_{x}\right]_{0}$ was used to minimise the effect produced by any discrepancies between $\left[I_{A}\right]_{0},\left[I_{B}\right]_{0}$ and $\left[I_{C}\right]_{0}$.

The traces for each different $T N_{x}$ were assayed 4 times, and the fluorescence of each reporter normalised with its mean maximum fluorescence during the assays.

\section{ASSOCIATED CONTENT}

Supporting information: Extended description of experimental and computational protocols, experimental controls and calibration curves, complete kinetic modelling of the handhold-mediated strand displacement mechanism, DNA sequences, and tables containing the fitting results of $k_{r}, k_{r}, K_{e q}, k_{t}, k_{b}, k_{m}$ and $k_{d}$ for all tested conditions at $25^{\circ}$ and $37^{\circ} \mathrm{C}$. This material is available free of charge via the Internet at http://pubs.acs.org.

All experimental files and MATLAB $®$ scripts used for its analysis are available for download through the file repository Zenodo at www.zenodo.org/record/4389212.

\section{AUTHOR INFORMATION}

\section{Corresponding Author}

Thomas E. Ouldridge - Department of Bioengineering and Centre for Synthetic Biology, Imperial College London, SW7 2AZ London, U.K. orcid.org/0000-0001-8114-8602. email: t.ouldridge@imperial.ac.uk

\section{Authors}

Javier Cabello-García - Department of Bioengineering and Centre for Synthetic Biology, Imperial College London, SW7 2AZ London, U.K. orcid.org/0000-0002-4025-1053

Wooli Bae - Department of Bioengineering and Centre for Synthetic Biology, Imperial College London, SW7 2AZ London, U.K. orcid.org/0000-0001-5396-3263

Guy Bart-Stan - Department of Bioengineering and Centre for Synthetic Biology, Imperial College London, SW7 2AZ London, U.K. orcid.org/0000-0002-5560-902X

\section{Author Contributions}

$\$$ These authors conceived the project and planned the experiments. + This author performed the experiments and analysed experimental data. All authors interpreted the results and co-wrote the paper.

\section{Funding Sources}

This work is part of a project that has received funding from the European Research Council (ERC) under the European Union's Horizon 2020 research and innovation programme (Grant agreement No. 851910).

\section{Notes}

The authors declare no competing interests.

\section{ACKNOWLEDGMENT}

The authors acknowledge financial support from the Royal Society (J.C.G. and T.E.O.), from the Royal Academy of Engineering via the RAE Chair in Emerging Technologies (GBS), and from the Engineering and Physical Sciences Research Council (WB) (grant agreement EP/P02596X/1).

\section{REFERENCES}

1. Chen, Y. J.; Dalchau, N.; Srinivas, N.; Phillips, A.; Cardelli, L.; Soloveichik, D.; Seelig, G., Programmable chemical controllers made from DNA. Nat. Nanotechnol. 2013, 8 (10), 755762.

2. Xin, L.; Zhou, C.; Duan, X.; Liu, N., A rotary plasmonic nanoclock. Nat. Commun. 2019, 10 (1), 5394.

3. Zhang, D. Y.; Seelig, G., Dynamic DNA nanotechnology using strand-displacement reactions. Nat. Chem. 2011, 3 (2), 10313.

4. Li, J.; Johnson-Buck, A.; Yang, Y. R.; Shih, W. M.; Yan, H.; Walter, N. G., Exploring the speed limit of toehold exchange with a cartwheeling DNA acrobat. Nat. Nanotechnol. 2018, 13 (8), 723-729.

5. Woods, D.; Doty, D.; Myhrvold, C.; Hui, J.; Zhou, F.; Yin, P.; Winfree, E., Diverse and robust molecular algorithms using reprogrammable DNA self-assembly. Nature 2019, 567 (7748), 366-372.

6. Hirose, Y.; Manley, J. L., RNA polymerase II and the integration of nuclear events. Genes Dev. 2000, 14 (12), 14151429.

$7 . \quad$ Crick, F., Central Dogma of Molecular Biology. Nature 1970, 227 (5258), 561-563.

8. Rodnina, M. V.; Gromadski, K. B.; Kothe, U.; Wieden, H. J., Recognition and selection of tRNA in translation. FEBS Lett. 2005, 579 (4), 938-942.

9. $\quad$ Tjivikua, T.; Ballester, P.; Rebek, J., Self-replicating system. Journal of the American Chemical Society 1990, 112 (3), 1249-1250. 
10. Sartori, P.; Leibler, S., Lessons from equilibrium statistical physics regarding the assembly of protein complexes. Proceedings of the National Academy of Sciences 2020, 117 (1), 114-120.

11. Ouldridge, T. E.; Rein Ten Wolde, P., Fundamental Costs in the Production and Destruction of Persistent Polymer Copies. Phys. Rev. Lett. 2017, 118 (15), 158103.

12. Poulton, J. M.; Ten Wolde, P. R.; Ouldridge, T. E., Nonequilibrium correlations in minimal dynamical models of polymer copying. Proceedings of the National Academy of Sciences of the United States of America 2019, 116 (6), 1946-1951. 13. Doye, J. P. K.; Wales, D. J., On potential energy surfaces and relaxation to the global minimum. The Journal of Chemical Physics 1996, 105 (18), 8428-8445.

14. Gorska, K.; Winssinger, N., Reactions Templated by Nucleic Acids: More Ways to Translate Oligonucleotide-Based Instructions into Emerging Function. Angew. Chem. Int. Ed. 2013, 52 (27), 6820-6843.

15. Di Pisa, M.; Seitz, O., Nucleic Acid Templated Reactions for Chemical Biology. ChemMedChem 2017, 12 (12), 872-882.

16. Michaelis, J.; Roloff, A.; Seitz, O., Amplification by nucleic acid-templated reactions. Org. Biomol. Chem. 2014, 12 (18), 2821-2833.

17. Dunn, K. E.; Dannenberg, F.; Ouldridge, T. E.; Kwiatkowska, M.; Turberfield, A. J.; Bath, J., Guiding the folding pathway of DNA origami. Nature 2015, 525 (7567), 82-6.

18. Zhang, D. Y.; Turberfield, A. J.; Yurke, B.; Winfree, E., Engineering entropy-driven reactions and networks catalyzed by DNA. Science 2007, 318 (5853), 1121-5.

19. Srinivas, N.; Ouldridge, T. E.; Sulc, P.; Schaeffer, J. M.; Yurke, B.; Louis, A. A.; Doye, J. P.; Winfree, E., On the biophysics and kinetics of toehold-mediated DNA strand displacement. Nucleic Acids Res. 2013, 41 (22), 10641-58.

20. Zhang, D. Y.; Winfree, E., Control of DNA Strand Displacement Kinetics Using Toehold Exchange. Journal of the American Chemical Society 2009, 131 (47), 17303-17314.

21. Srinivas, N.; Parkin, J.; Seelig, G.; Winfree, E.; Soloveichik, D., Enzyme-free nucleic acid dynamical systems. Science 2017, 358 (6369).

22. Green, A. A.; Kim, J.; Ma, D.; Silver, P. A.; Collins, J. J.; Yin, P., Complex cellular logic computation using ribocomputing devices. Nature 2017, 548 (7665), 117-121.

23. Chen, X., Expanding the rule set of DNA circuitry with associative toehold activation. Journal of the American Chemical Society 2012, 134 (1), 263-71.

24. Genot, A. J.; Zhang, D. Y.; Bath, J.; Turberfield, A. J., Remote toehold: a mechanism for flexible control of DNA hybridization kinetics. J. Am. Chem. Soc. 2011, 133 (7), 2177-82. 25. Lanzmich, S. A. Replication in Early Evolution. LudwigMaximilians-Universität, 2016.

26. O'Flaherty, D. K.; Zhou, L. J.; Szostak, J. W., Nonenzymatic Template-Directed Synthesis of Mixed-Sequence 3 '-NP-DNA up to 25 Nucleotides Long Inside Model Protocells. Journal of the American Chemical Society 2019, 141 (26), 1048110488.

27. Lincoln, T. A.; Joyce, G. F., Self-Sustained Replication of an RNA Enzyme. Science 2009, 323 (5918), 1229-1232.

28. Schulman, R.; Yurke, B.; Winfree, E., Robust selfreplication of combinatorial information via crystal growth and scission. Proceedings of the National Academy of Sciences of the United States of America 2012, 109 (17), 6405-6410.

29. Zhou, L.; Kim, S. C.; Ho, K. H.; O'Flaherty, D. K.; Giurgiu, C.; Wright, T. H.; Szostak, J. W., Non-enzymatic primer extension with strand displacement. eLife 2019, 8, e51888.

30. Osuna Gálvez, A.; Bode, J. W., Traceless Templated Amide-Forming Ligations. Journal of the American Chemical Society 2019, 141 (22), 8721-8726.

31. Sadownik, J. W.; Mattia, E.; Nowak, P.; Otto, S., Diversification of self-replicating molecules. Nat. Chem. 2016, 8 (3), 264-9.

32. Haley, N. E. C.; Ouldridge, T. E.; Mullor Ruiz, I.; Geraldini, A.; Louis, A. A.; Bath, J.; Turberfield, A. J., Design of hidden thermodynamic driving for non-equilibrium systems via mismatch elimination during DNA strand displacement. Nat. Commun. 2020, 11 (1), 2562.

33. Moreira, B. G.; You, Y.; Owczarzy, R., Cy3 and Cy5 dyes attached to oligonucleotide terminus stabilize DNA duplexes: predictive thermodynamic model. Biophys. Chem. 2015, 198, 3644.

34. Wang, B.; Thachuk, C.; Ellington, A. D.; Winfree, E.; Soloveichik, D., Effective design principles for leakless strand displacement systems. Proceedings of the National Academy of Sciences 2018, 115 (52), E12182-E12191.

35. SantaLucia, J., Jr.; Hicks, D., The thermodynamics of DNA structural motifs. Annu Rev Biophys Biomol Struct 2004, 33, 415-40.

36. Stellwagen, N. C., Apparent pore size of polyacrylamide gels: Comparison of gels cast and run in Tris-acetate-EDTA and Tris-borate-EDTA buffers. Electrophoresis 1998, 19 (10), 15421547.

37. Wolfe, B. R.; Porubsky, N. J.; Zadeh, J. N.; Dirks, R. M.; Pierce, N. A., Constrained Multistate Sequence Design for Nucleic Acid Reaction Pathway Engineering. J. Am. Chem. Soc. 2017, 139 (8), 3134-3144.

38. Genot, A. J.; Bath, J.; Turberfield, A. J., Combinatorial Displacement of DNA Strands: Application to Matrix Multiplication and Weighted Sums. Angew. Chem. Int. Ed. 2013, 52 (4), 1189-1192.

39. Gomez, M. A. R.; Ibba, M., Aminoacyl-tRNA synthetases. RNA 2020, 26 (8), 910-936.

40. Deshpande, A.; Ouldridge, T. E., Optimizing enzymatic catalysts for rapid turnover of substrates with low enzyme sequestration. ArXiv 2019, 1905.00555.

41. Kanoatov, M.; Krylov, S. N., DNA Adsorption to the Reservoir Walls Causing Irreproducibility in Studies of ProteinDNA Interactions by Methods of Kinetic Capillary Electrophoresis. Anal. Chem. 2011, 83 (20), 8041-8045.

42. Srinivas, N.; Parkin, J.; Seelig, G.; Winfree, E.; Soloveiehile, D., Enzyme-free nucleic acid dynamical systems. Science 2017, 358 (6369). 


\section{Handhold-mediated DNA strand displacement}

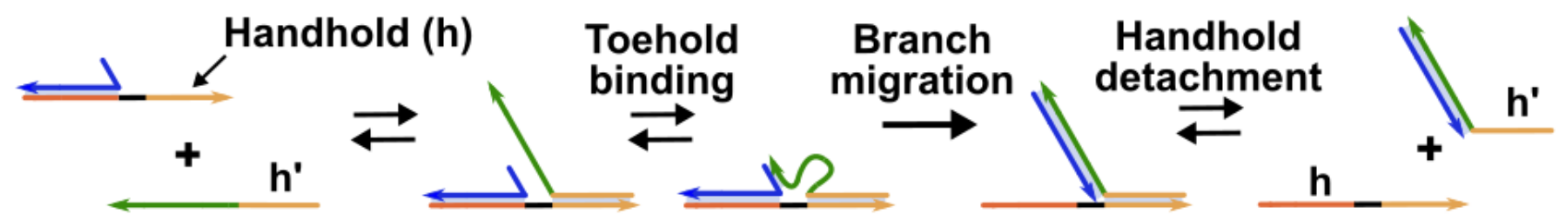

Selective DNA duplex production out of equilibrium

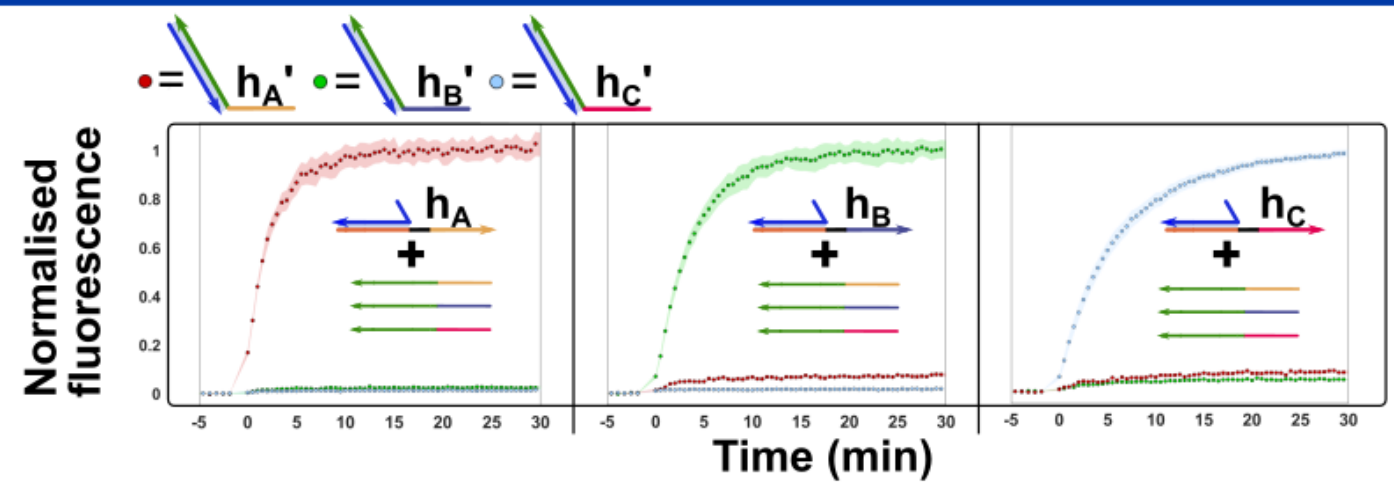

"For Table of Contents Only" 


\section{Handhold-Mediated Strand Displacement: A Nucleic Acid- Based Mechanism for Generating Far-from-Equilibrium Assemblies Through Templated Reactions}

\section{Supplementary Information}

Javier Cabello-Garcia Wooli Bae, Guy-Bart V. Stan and Thomas E. Ouldridge

\section{Supplementary Notes}

Supplementary Note I: General procedure for measuring reaction kinetics

Supplementary Note II: Experimental procedure for the kinetic analysis of handhold detachment .8

Supplementary Note III: Selective production of complexes far-from-equilibrium ........................ 12

Supplementary Note IV: Experimental protocol for fluorescence calibration curves.....................14

Supplementary Note V: Fitting procedure for fluorescence calibration curves ...........................15

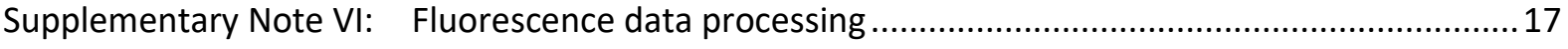

Supplementary Note VII: Additional fluorescence processing .............................................. 19

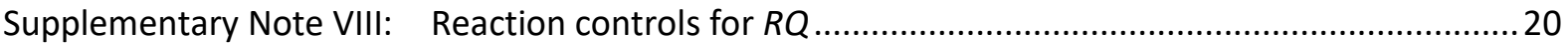

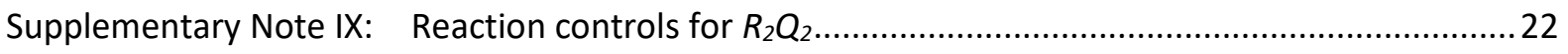

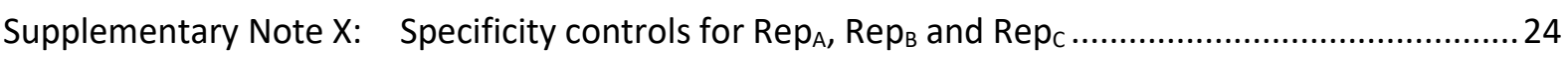

Supplementary Note XI: Implementation of handhold-mediated strand displacement reaction

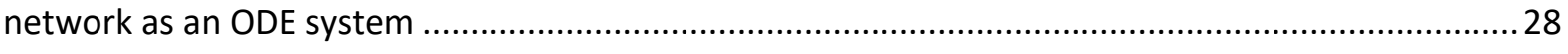

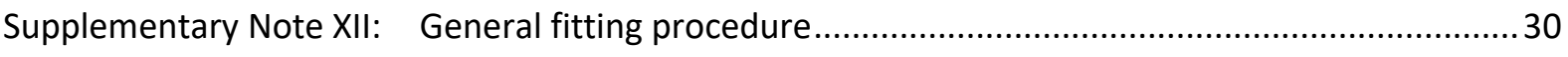

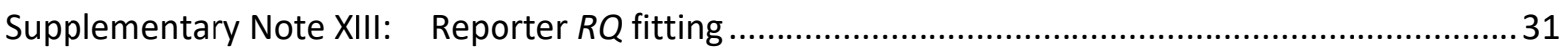

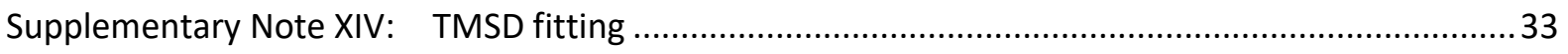

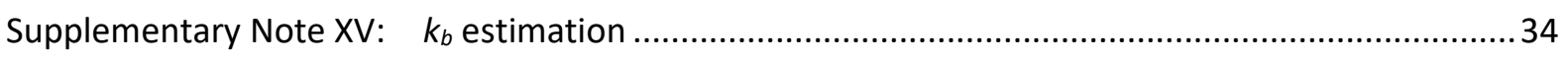

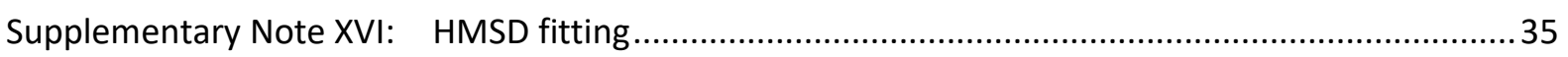

Supplementary Note XVII: Fitting of the kinetic analysis of handhold detachment .......................43

Supplementary Note XVIII: Derivation of HMSD bimolecular rate (equation 4) ........................... 45

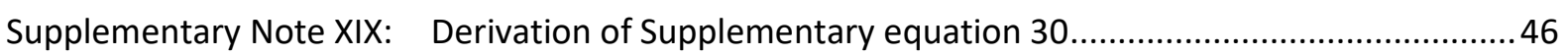

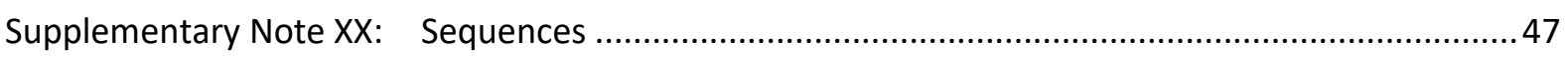




\section{Supplementary Figures}

Supplementary Figure 1 | Example of reporter complex $R Q$ reporter characterisation.

Supplementary Figure 2 | Example of a handhold-mediated strand displacement characterisation experiment.

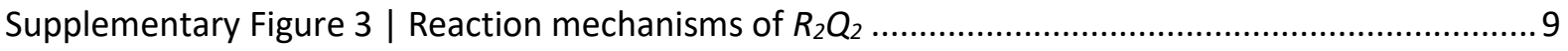

Supplementary Figure 4 | Example of the kinetic analysis of handhold detachment........................... 10

Supplementary Figure 5 | Kinetic analysis in PAGE buffer condition................................................. 11

Supplementary Figure 6 | Example of raw experimental data from the production of complex $I_{A} T$.. 13

Supplementary Figure 7 | RQ reporter complex (Cy3) calibration curve at $25^{\circ} \mathrm{C}$. Gain $2251 \ldots \ldots \ldots \ldots . . .15$

Supplementary Figure 8| RQ reporter complex (Cy3) calibration curve at $37^{\circ} \mathrm{C}$. Gain $2336 \ldots \ldots \ldots \ldots . .15$

Supplementary Figure $9 \mid \mathrm{RQ}$ reporter complex (Cy3) calibration curve at 25 degrees. Gain $2230 \ldots . . .16$

Supplementary Figure $10 \mid \mathrm{R}_{2} \mathrm{Q}_{2}$ reporter complex (Cy5) calibration curve at 25 degrees. Gain 281616

Supplementary Figure 11 | Example of fluorescence data processing ............................................... 18

Supplementary Figure 12 | Reporter $R Q$ control, proving the absence of leak when mixed with

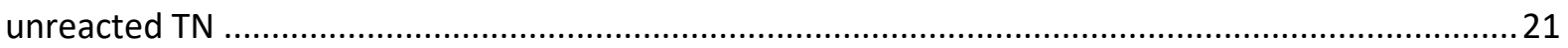

Supplementary Figure 13 | Reporter $R Q$ control using / without $T$-complementary, proving the absence of reaction when using an / strand that is only handhold complementary............................21

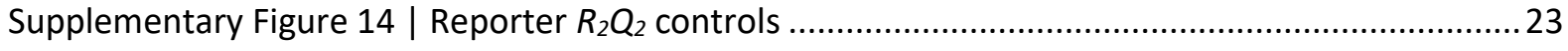

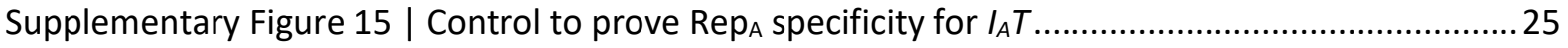

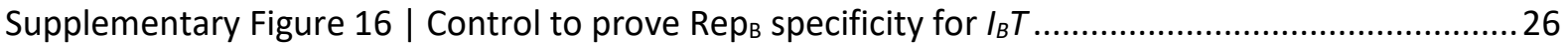

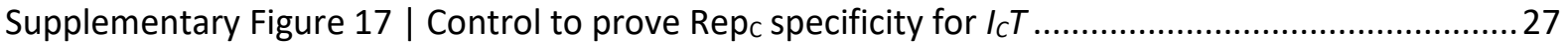

Supplementary Figure $18 \mid$ Inferred $2^{\text {nd }}$ order rate constants for handhold-mediated strand displacement.

Supplementary Figure 19 | Inferred $2^{\text {nd }}$ order rate constants for I-strands without handhold complementarity......

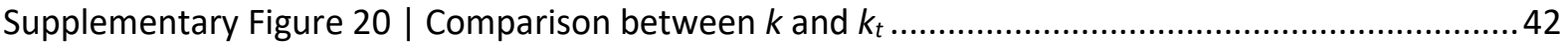

Supplementary Figure 21 | Triggering of reporter $R_{2} Q_{2}$ in the absence of handhold........................ 44 


\section{Supplementary Tables}

Supplementary Table 1 | Detailed experimental procedure for reporter $R Q$ characterisation.

Supplementary Table 2 | Detailed experimental procedure for toehold/handhold-mediated strand displacement characterisation.

Supplementary Table 3 | Detailed experimental procedure for the kinetic analysis of handhold detachment.

Supplementary Table 4 | Detailed experimental procedure to produce far-from-equilibrium complexes.....

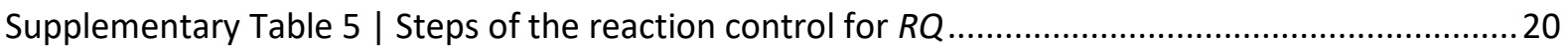

Supplementary Table 6 | Steps of the control for / without $T$-complementarity................................2 20

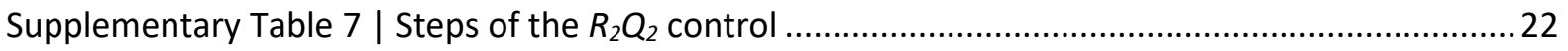

Supplementary Table 8 | Steps of the selectivity controls for Rep complexes .....................................24

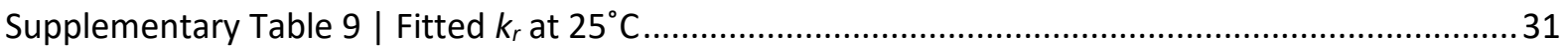

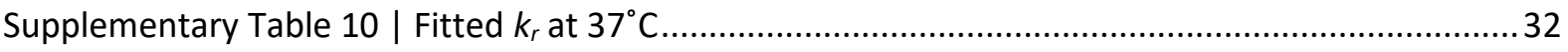

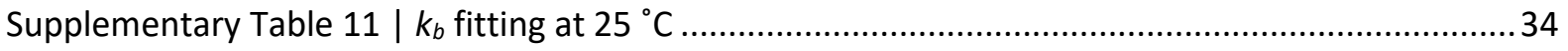

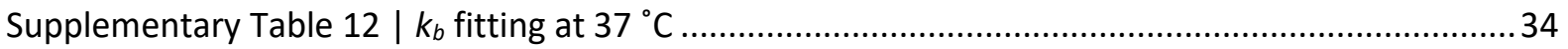

Supplementary Table 13 | Estimated $\Delta \mathrm{G}_{h b}$ and $k_{d}$ for each handhold at $25^{\circ} \mathrm{C}$...................................36

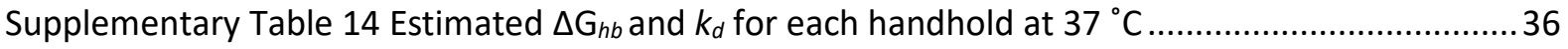

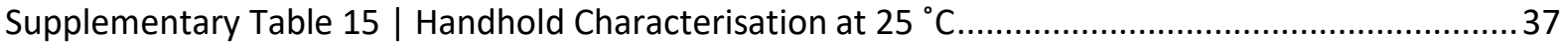

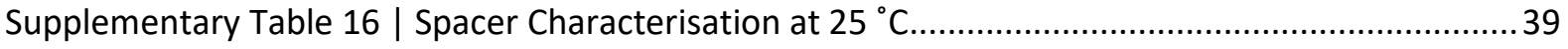

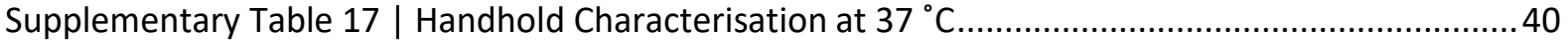

Supplementary Table 18 | Fitting results from the kinetic analysis of handhold detachment .............43

Supplementary Table 19 | Fitting results from the kinetic analysis of handhold detachment in the

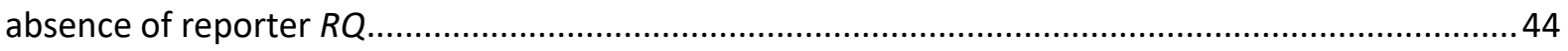

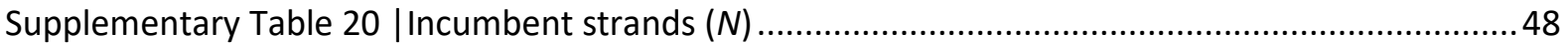

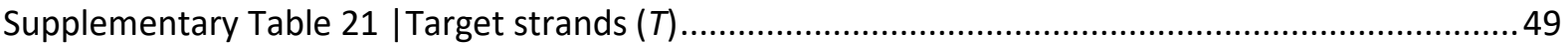

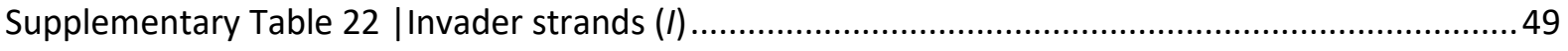

Supplementary Table 23 | Reporter strands ..................................................................................... 49 


\section{Supplementary Note I: General procedure for measuring reaction kinetics}

All experiments contained at least one negative control (buffer only) and one positive control per reporter used, which allowed correction of changes in fluorescence by temperature and reaction volume. Positive controls contained a variable concentration of the reporter's fluorophore-labelled strand in the experimental buffer (TAE $1 \times, 1 \mathrm{M} \mathrm{NaCl})$. The volume contained in the controls changed consistently with the volume of the main assays. The function of the controls during data analysis is further described in Supplementary Note VI.

All measurements consisted of at least 10 data points. The number of measurements per experiment depended on the class of characterisation experiment; However, they all followed a similar structure that helped to quantify all the species in the experimental solution from the steady-state fluorescence, as outlined below. Specific procedures for each type of experiment are collected in Supplementary Tables $\mathbf{1}$ and 2. Examples of their fluorescence traces are shown in Supplementary Figures $\mathbf{1}$ and $\mathbf{2}$. For convenience, in all Supplementary Tables and Figures, we report the quantity of each species in the reaction volume at any time as the concentration that would be present if the same amount was solvated in a reaction volume of $200 \mu \mathrm{L}$.

Measurement 1. Fluorescence baseline produced by residual fluorescence of $R Q$ :

The experiments started by mixing $10 \mu \mathrm{L}$ of a standard solution of $R Q(300 \mathrm{nM})$ with experimental buffer. A measurement was made after 30 mins of incubation at the experimental temperature, when the fluorescence value of the positive controls became stable.

Measurement 2. Estimation of $[N]_{\text {excess: }}$

$10 \mu \mathrm{L}$ of a standard solution of $T N(200 \mathrm{nM})$ were added to the solution, except during Reporter characterisations. After adding $T N$, the excess of $N$ resultant from the annealing of $T N\left([N]_{\text {excess}}\right)$ immediately reacted with $R Q$, producing an increment of the fluorescence baseline (See Supplementary Note $\mathbf{X I}$ ). The new baseline was measured and used to infer $[N]_{\text {excess. }}$.

\section{Measurement 3. Experimental kinetics:}

$50 \mu \mathrm{L}$ of a solution with the reaction trigger (/ or N/IT) was added for a final reaction volume of $200 \mu \mathrm{L}$. Intended reagent concentrations at $200 \mu \mathrm{L}$ were $[R Q]_{0}=15 \mathrm{nM},[N]_{0}=10 \mathrm{nM}$ and $[/]_{0}$ ranging from 4 to $8 \mathrm{nM}$ unless specified otherwise. The system kinetics were measured after the injection, when possible until the reaction reached equilibrium. For kinetics that took more than 2 hours to reach equilibrium, several systems, with their respective controls, were run in parallel in a single 96 -well plate. The kinetics of the experiments in a 96-well plate were always measured for at least 100 hours. After measuring, the plate was sealed and incubated for 1 month at the experimental temperature, and preserved from light, to wait for the reaction to reach equilibrium.

Measurement 4. Estimation of reaction trigger concentration from the steady-state fluorescence:

After the reaction reached equilibrium, a measurement recorded the fluorescence plateau. The total amount of reaction trigger added was inferred from this measurement. Some systems had such slow displacement rate that they did not reach equilibrium after 1 month (proximal toehold $=0 \mathrm{nt}$; short handholds). For the slow-rate systems, trigger concentration was inferred from the mean concentration of all the experiments that reached equilibrium in the same plate. All experiments in a plate were triggered with the same aliquot of reaction trigger, so significant variability was not expected between experiments. Experiments that reacted till at least $80 \%$ of the highest concentration of trigger measured in that same plate were considered to have reached equilibrium. 
Measurement 5. Saturation and quantification of the rest of the strands:

Finally, the experimental solution was sequentially saturated. High concentrations of single strands were added to trigger unreacted substrates and infer their actual initial concentrations from the resultant change in fluorescence. For example, to quantify $[T N]_{0}$, all the $T N$ in the solution was triggered with $10 \mu \mathrm{L}$ of a saturating solution of $I_{h c}$ (Measurement $\left.5 \mathrm{i}\right)$ ). [RQ $]_{0}$ was estimated similarly with a saturating solution of $N$ (Measurement $5 \mathrm{ii})$ ).

Supplementary Table 1| Detailed experimental procedure for reporter $R Q$ characterisation.

Due to the high rate of the reaction, the four iterations of each experiment were triggered and measured individually. The triggering species was a solution of $N / I T$. Since no $T N$ was added, no $[N]_{\text {excess }}$ existed, and no measurement 2 was needed.

\begin{tabular}{|c|c|c|c|c|}
\hline Steps & Addition & Purpose & $\begin{array}{l}\text { Expected concentrations in } 200 \mu \mathrm{L} \\
(\mathrm{nM})\end{array}$ & $\begin{array}{c}\text { Volume } \\
(\mu \mathrm{L})\end{array}$ \\
\hline Measurement 1 & $\begin{array}{c}10 \mu \mathrm{L} R Q(300 \mathrm{nM}) \\
140 \mu \mathrm{L} \text { Buffer }\end{array}$ & $\begin{array}{c}\text { Estimate residual } \\
\text { fluorescence baseline }\end{array}$ & $R Q: 15$ & 150 \\
\hline Measurement 3 & $\begin{array}{l}\text { [45-20] } \mu \mathrm{L} N / I T(40 \mathrm{nM}) \\
\quad+[5-30] \mu \mathrm{L} \text { Buffer }\end{array}$ & Experimental kinetics & Evolve with time & 200 \\
\hline Measurement 4 & - & Estimate $[N]_{0}$ & $R Q:[6-11] ; R N:[9-4] ; / T:[9-4] ;$ & 200 \\
\hline Measurement $5 \mathrm{ii)}$ & $10 \mu \mathrm{L} N(400 \mathrm{nM})$ & Estimate $[R Q]_{0}$ & $R N: 15 ; / T:[9-4] ; N:[14-9]$ & 210 \\
\hline
\end{tabular}

Supplementary Table 2| Detailed experimental procedure for toehold/handhold-mediated strand displacement characterisation.

The four trajectories of each reaction system and their controls were triggered by mechanical injection and measured simultaneously. To characterise the handhold-mediated strand displacement, the reaction was triggered with $I_{h c}$. To characterise the toehold rate $k_{t}, I_{w c}$ was used. The species $I_{w c}$ have, instead of the handhold-complementary domain, a random sequence of the same length.

\begin{tabular}{|c|c|c|c|c|}
\hline Steps & Addition & Purpose & $\begin{array}{l}\text { Expected concentrations in } 200 \mu \mathrm{L} \\
(\mathrm{nM})\end{array}$ & $\begin{array}{l}\text { Volume } \\
(\mu \mathrm{L})\end{array}$ \\
\hline Measurement 1 & $\begin{array}{c}10 \mu \mathrm{L} R Q(300 \mathrm{nM}) \\
130 \mu \mathrm{L} \text { Buffer }\end{array}$ & $\begin{array}{l}\text { Estimate residual } \\
\text { fluorescence baseline }\end{array}$ & $R Q: 15$ & 140 \\
\hline Measurement 2 & $\begin{array}{c}10 \mu \mathrm{L} T N(200 \mathrm{nM})+ \\
N \sim 20 \mathrm{nM}\end{array}$ & Estimate $[N]_{\text {excess }}$ & $R Q: 14 ; T N: 10 ; R N: \sim 1$ & 150 \\
\hline Measurement 3 & $\begin{array}{c}{[45-20] \mu \mathrm{L} /(40 \mathrm{nM})+} \\
{[5-30] \mu \mathrm{L} \text { Buffer }}\end{array}$ & System kinetics & Evolve with time & 200 \\
\hline Measurement 4 & - & Estimate $\left[I_{0}+[N]_{\text {excess }}\right.$ & $R Q:[5-10] ; T N:[1-6] ; R N:[10-5] ; / T:[9-4]$ & 200 \\
\hline Measurement 5 i) & $10 \mu \mathrm{L} / \mathrm{hc}(400 \mathrm{nM})$ & Estimate $[N]_{0}$ & $R Q: 4 ; R N: 11 ; I T: 10 ; I:[19-14]$ & 210 \\
\hline Measurement 5 ii) & $10 \mu \mathrm{L} N(400 \mathrm{nM})$ & Estimate $[R Q]_{0}$ & $R N: 15 ; I T: 10 ; I:[19-14] ; N: 16$ & 220 \\
\hline
\end{tabular}




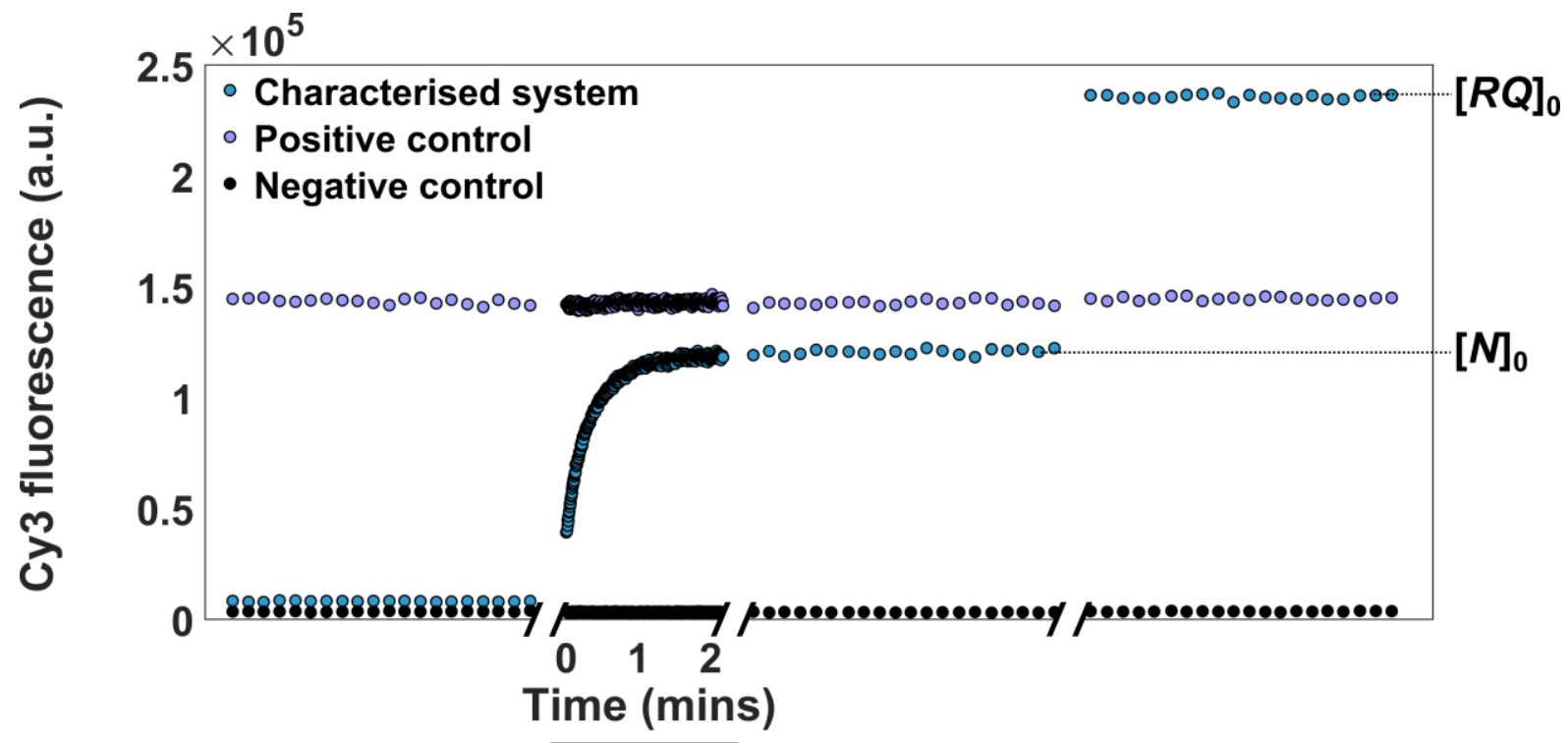

\section{Meas. 1 Meas. 3 Meas. $4 \quad$ Meas. 5 ii)}

Supplementary Figure 1| Example of reporter complex $R Q$ reporter characterisation.

Raw data from the triggering reaction of $R Q$ with an $N / I T$ complex with a $5 \mathrm{nt}$ handhold, $8 \mathrm{nt}$ spacer and $7 \mathrm{nt}$ toehold at $25^{\circ} \mathrm{C}\left(5 / 8 / 7 \_25\right)$. Measurement 1 contains the baseline of the experiment produced by $15 \mathrm{nM}$ of quenched $R Q$. In measurement 3 , a variable amount of trigger $N / I T$ was added, between 9 and $4 \mathrm{nM}$ ( $8 \mathrm{nM}$ in this case). After the reaction finished, the total amount of trigger $N / I T$ was estimated from measurement 4 . After that, $20 \mathrm{nM}$ of $N$ was added to trigger the rest of $R Q$ and quantify it in measurement $5 \mathrm{ii}$. All concentrations are defined for a reaction volume of $200 \mu \mathrm{L}$. The positive controls contained more than $10 \mathrm{nM}$ of the Cy3-labelled reporter strand. The negative controls consisted of only the experimental buffer. 


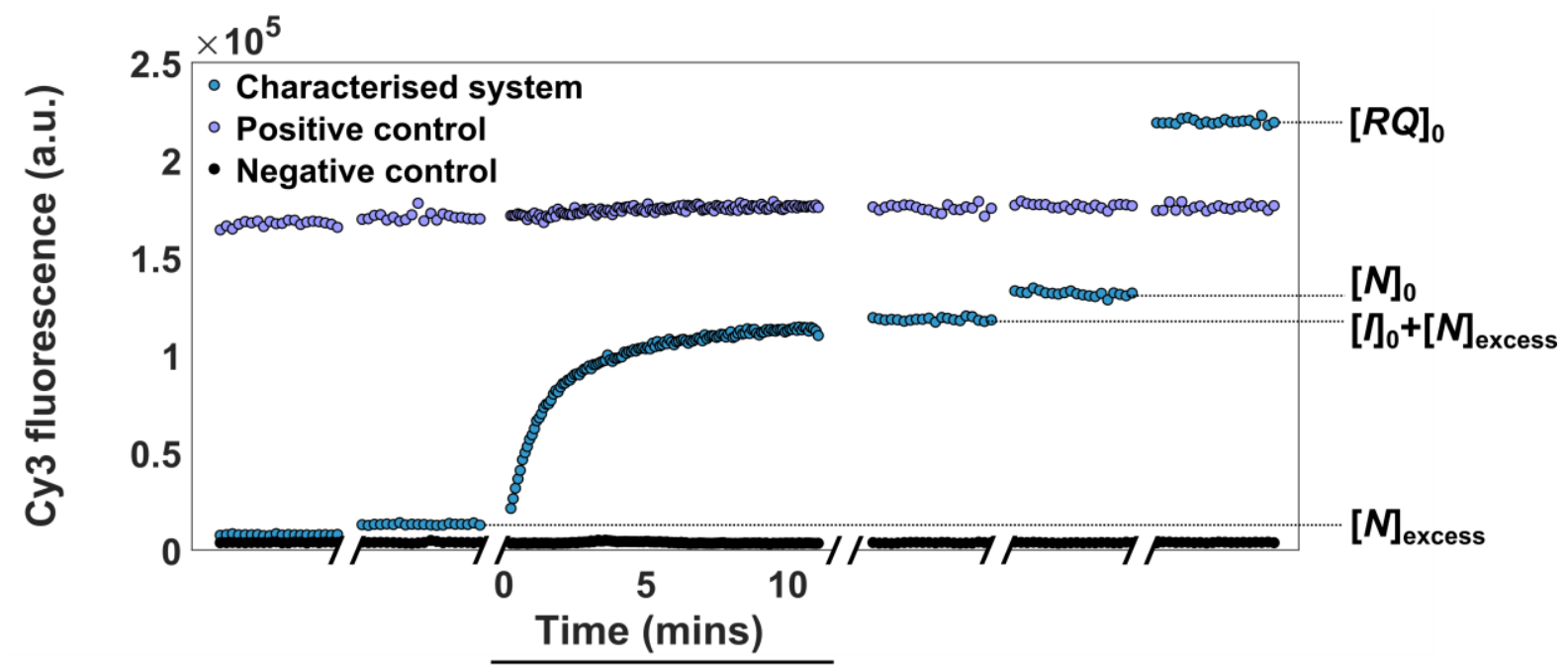

\section{Meas.1 Meas.2 Meas.3 Meas.4 Meas.5 i) ii)}

\section{Supplementary Figure 2| Example of a handhold-mediated strand displacement characterisation} experiment.

Raw data from the reaction system with a $7 \mathrm{nt}$ handhold, $2 \mathrm{nt}$ spacer and $5 \mathrm{nt}$ toehold at $25^{\circ} \mathrm{C}$ $\left(7 / 2 / 5\right.$ _25) triggered with $I_{h c}$. Measurement 1 contains the baseline of the experiment produced by 15 $\mathrm{nM}$ of quenched $R Q$. Then, $10 \mathrm{nM}$ of $T N$ was added. $[N]_{\text {excess }}$ was inferred from the annealing increase the baseline, which was recorded in measurement 2 . In measurement 3 , a variable amount of trigger $I_{h c}$ was added, between 9 and $4 \mathrm{nM}(8 \mathrm{nM}$ in this case). After the reaction finished, measurement 4 was used to estimate the total amount of trigger $I_{h c}$. Measurement 5 i) saturated the solution with 20 $\mathrm{nM}$ of $I_{\mathrm{hc}}$ to estimate the total amount of $N$ in the solution. After that, $20 \mathrm{nM}$ of $N$ was added to trigger the rest of $R Q$ and quantify it in measurement $5 \mathrm{ii}$ ). All concentrations are defined for a reaction volume of $200 \mu \mathrm{L}$. The positive controls contained more than $10 \mathrm{nM}$ of the Cy3-labelled reporter strand. The negative controls consisted of only the experimental buffer. 


\section{Supplementary Note II: Experimental procedure for the kinetic analysis of handhold detachment}

The design of detachment experiments had to be modified to minimise $R_{2} Q_{2}$ leak reactions (Supplementary Figure 3). The triggering mechanism for the reporter $R_{2} Q_{2}$ is composed of an associative toehold with an 8-nt distal toehold and a 2-nt proximal toehold. The distal toehold is complementary to the handhold-complementary domain of $I_{\mathrm{hc}}$ and is unable to bind $R_{2} Q_{2}$ until IT detaches. However, the proximal toehold is complementary with a $3^{\prime}$ additional domain in $T$. This 2-nt toehold-mediated displacement reaction can always take place when $R_{2} Q_{2}$ and $T$ are together in the solution.

To reduce the time that $R_{2} Q_{2}$ and the $T$ strand were together, $R_{2} Q_{2}$ was added as the trigger of the reaction. Steps of the experiments and an example of the fluorescence traces are shown in Supplementary Table 3 and Supplementary Figure 4. The modification required the addition of a new control to the experiment. Besides the negative control and the positive controls for Cy5 and Cy3 fluorescence, a $4^{\text {th }}$ control was added to quantify the residual fluorescence resultant produced by quenched $R_{2} Q_{2}$. This "quenching control" was composed of $15 \mathrm{nM}$ of the reporter complex $R_{2} Q_{2}$ from the same aliquot used during the experiments. This control was eventually saturated with IT complex to measure the total fluorescence and calculate the efficiency of quenching for $R_{2} Q_{2}$. Then, the quenching efficiency was used to infer the fluorescence baseline for the experimental trajectories.

\section{Control without $R Q$ reporter complex:}

The signal of reporter $R Q$ was used to infer the concentrations of all the species in the solution. The Cy3 signal had a higher signal-to-noise ratio and informed about $[N]_{\text {excess, }}$ which is relevant for the calculation of $K_{\text {eq }}$ (eq.15). To prove that $R Q$ did not modify $K_{\text {eq }}$, a subset of the reactions $(7,8,9$ and 10 nt handhold) was repeated without including $R Q$ in the solution. In this control without $R Q$, the concentration of each species was inferred only from the signal produced by $R_{2} Q_{2}$. The use of $R_{2} Q_{2}$ resulted in a more inexact determination of the species concentration, like $N_{\text {excess, }}$ which could not be quantified (it was assumed to be 0). Results from this control, collected in Supplementary Table 19, showed that rate saturation still occurred for handholds shorter than 9 nt. The obtained $K_{\text {eq }}$ slightly differed, but that effect could be attributed to the limitations during the quantification of the assay species.

\section{Control at PAGE conditions:}

To avoid excessive heating, during PAGE electrophoresis, $\mathrm{NaCl}$ was exchanged with a smaller amount of $\mathrm{MgCl}$. The expected decrease of Gibbs free energy for the magnesium buffer condition is approximately $0.1 \mathrm{kcal} /$ basepair.mol ${ }^{1,2}$. A new control was assayed to demonstrate that handhold detachment is comparable between both buffer conditions. The kinetic analysis experiment described in Supplementary Table 3 was repeated for a buffer condition of $12.5 \mathrm{mM}[\mathrm{MgCl} 2]$ in 0.5 X TAE for a subset of the reactions $(7,8,9,10$ and 20 nt handhold). Due to the presence of divalent cations, the behaviour of the reporter system could change, hindering reaction species quantification and comparison between conditions. The fluorescence signal of $R 2 Q 2$ reaction traces was normalised to the value obtained in measurement 5 , except for the condition with a 20 nt handhold, which was normalised with measurement 6 . The fluorescence signal was not processed with the positive controls either. The obtained trajectories are shown in Supplementary Figure 5. The result of the control show qualitatively the same behaviour observed in the $\mathrm{NaCl}$ buffer condition. The traces of the systems with 7 and $8 \mathrm{nt}$ handhold have comparable rates, but the initial reaction rate decreases for 9 and $10 \mathrm{nt}$. Also, the system with a $20 \mathrm{nt}$ handhold shows no detachment at all. 
Supplementary Table 3| Detailed experimental procedure for the kinetic analysis of handhold detachment.

The four trajectories of each reaction system and their controls were triggered by mechanical injection and measured simultaneously. All the tested $T N$ contained $3 \mathrm{nt}$ proximal toeholds and a $2 \mathrm{nt}$ spacer.

\begin{tabular}{|c|c|c|c|c|}
\hline Conditions & Addition & Purpose & $\begin{array}{l}\text { Expected concentrations in } \\
200 \mu \mathrm{L} \\
(\mathrm{nM})\end{array}$ & $\begin{array}{c}\text { Volume } \\
(\mu \mathrm{L})\end{array}$ \\
\hline Measurement 1 & $\begin{array}{c}10 \mu \mathrm{L} R Q(300 \mathrm{nM}) \\
80 \mu \mathrm{L} \text { Buffer }\end{array}$ & $\begin{array}{l}\text { Estimate residual } \\
\text { fluorescence baseline }\end{array}$ & $R Q: 15$ & 90 \\
\hline Measurement 2 & $\begin{array}{c}10 \mu \mathrm{L} T N(200 \mathrm{nM})+ \\
N \sim 20 \mathrm{Nm}\end{array}$ & Estimate $[N]_{\text {excess }}$ & $R Q: 14 ; T N: 10 ; R N: \sim 1$ & 100 \\
\hline Measurement 3 & $\begin{array}{c}{[45-20] \mu \mathrm{L} \operatorname{lnc}(40 \mathrm{nM})+} \\
{[5-30] \mu \mathrm{L} \text { Buffer }}\end{array}$ & $\begin{array}{l}\text { Trigger } T N \text { and estimate } \\
{\left[[]_{0}+[N]_{\text {excess }}\right.}\end{array}$ & $R Q:[5-10] ; T N:[1-6] ; R N:[10-5] ; / T:[9-4]$ & 150 \\
\hline Measurement 4 & $\begin{array}{l}50 \mu \mathrm{L} R_{2} Q_{2}(60 \mathrm{nM}) \text {. } \\
\text { Reporter is also added to } \\
\text { the quenched control. }\end{array}$ & $R_{2} Q_{2}$ kinetics & Evolve with time & 200 \\
\hline Measurement 5 & - & Estimate $[I T]^{\text {Total }}$ & $\begin{array}{c}R Q:[5-10] ; T N:[1-6] ; R N:[10-5] ; \\
R_{2} / T:[9-4] ; R_{2} Q_{2}:[6-11]\end{array}$ & 200 \\
\hline Measurement 6 & $10 \mu \mathrm{L} / \mathrm{hc}(400 \mathrm{nM})$ & Estimate $[N]_{0}$ & $R Q: 4 ; R N: 11 ; R_{2} / T: 10 ; R_{2} Q_{2}: 5 ; l:[19-14]$ & 210 \\
\hline Measurement 7 & $10 \mu \mathrm{L} T(400 \mathrm{nM})$ & Estimate $\left[R_{2} Q_{2}\right]_{0}$ & $R Q: 4 ; R N: 11 ; / T^{\sim}[10] ; R_{2} / T: 15$ & 220 \\
\hline
\end{tabular}

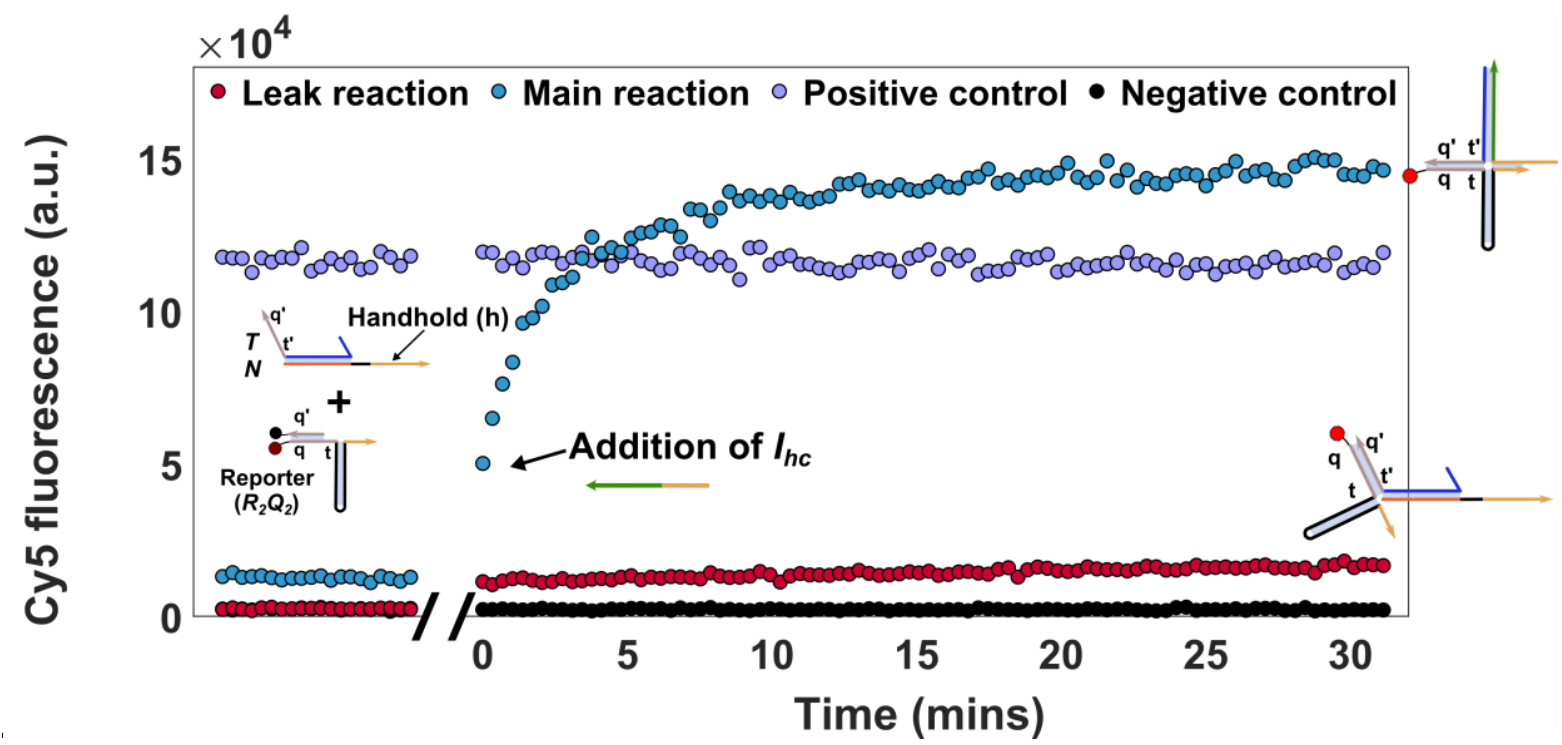

Supplementary Figure 3 | Reaction mechanisms of $R_{2} Q_{2}$

Raw data from $R_{2} Q_{2}$ characterisation experiments. Main reaction) Intended reaction mechanism for $R_{2} Q_{2}$. A solution of $15 \mathrm{nM}$ of $R_{2} Q_{2}$ is triggered by $10 \mathrm{nM}$ of $N / I T$. The detached $I T$ triggered an associative toehold reaction with a rate of $7.14 \pm 0.23 .10^{5} \mathrm{M}^{-1} \mathrm{~s}^{-1}\left(k_{r 2}\right.$; Supplementary Figure 21). Leak reaction) Leak produced after adding $15 \mathrm{nM}$ of $R_{2} Q_{2}$ to a solution with $10 \mathrm{nM} T N$. The mere presence of $R_{2} Q_{2}$ and $T$ in the same solution triggered a $2 \mathrm{nt}$ toehold-mediated strand displacement with an estimated rate of $4.66 \pm 0.89 .10^{2} \mathrm{M}^{-1} \cdot \mathrm{s}^{-1}$ (Rate inferred from the mean of the assayed $2 \mathrm{nt}$ toehold-mediated strand displacement reactions at $25^{\circ} \mathrm{C}$ ). All concentrations are defined for a reaction volume of $200 \mu \mathrm{L}$. The positive controls contained more than $10 \mathrm{nM}$ of the Cy5-labelled reporter strand. The negative controls consisted of only the experimental buffer. 


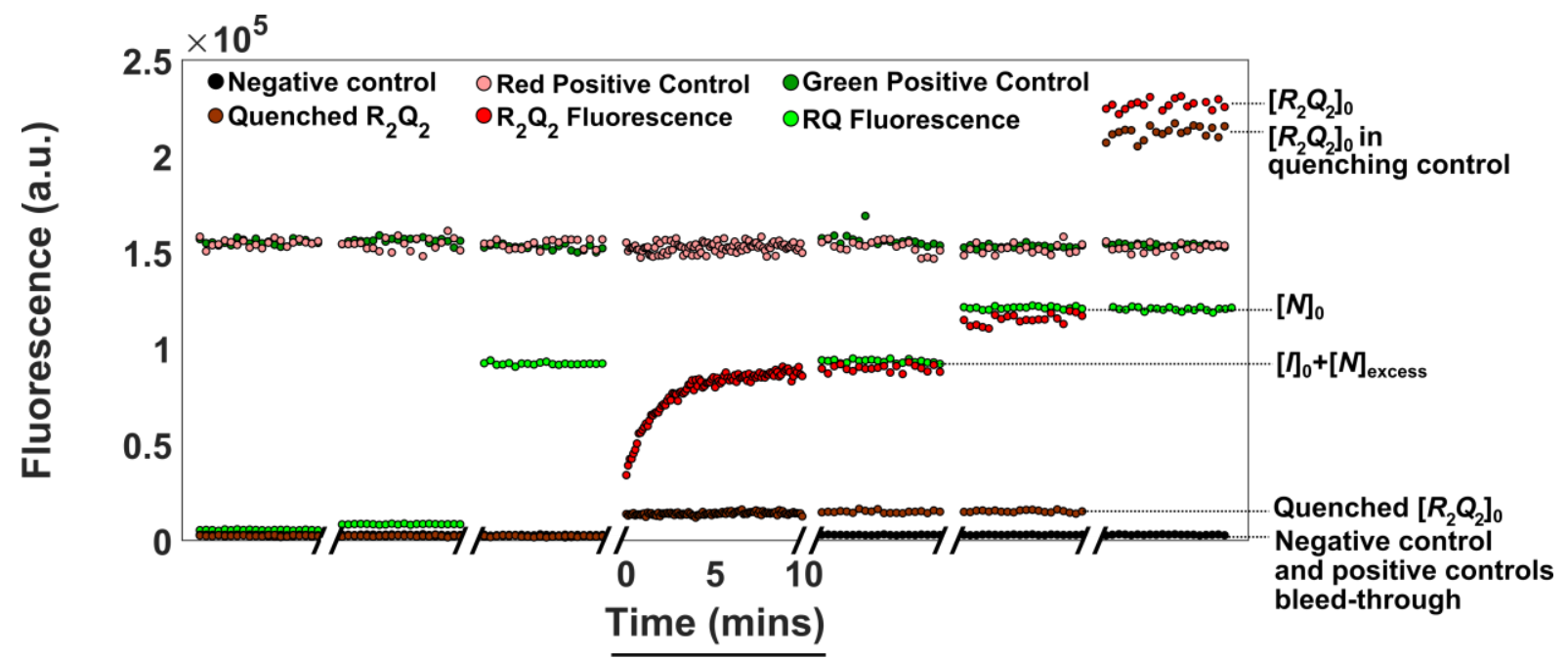

Meas. 1 Meas.2 Meas.3 Meas.4 Meas.5 Meas.6 Meas.7

Supplementary Figure 4 | Example of the kinetic analysis of handhold detachment

Raw data from the reaction system with a $7 \mathrm{nt}$ handhold, $2 \mathrm{nt}$ spacer and $3 \mathrm{nt}$ toehold at $25^{\circ} \mathrm{C}$ (7/2/3_25) triggered with $I_{\text {hc. }}$. $T$ strands in these experiments always contain an extra domain at $3^{\prime}$ that triggers reporter $R_{2} Q_{2}$. Measurement 1 contains the baseline of the experiment produced by $15 \mathrm{nM}$ of quenched $R Q$. Then, $10 \mathrm{nM}$ of $T N$ was added. $[N]_{\text {excess }}$ was inferred from the increase in Cy3 baseline, which was recorded in measurement 2 . After that, a variable amount of trigger $I_{h c}$ was added, between 9 and $4 \mathrm{nM}\left(8 \mathrm{nM}\right.$ in this case). After the reaction finished, measurement 3 estimated $[I]_{0}$, which is equal to the total amount of produce IT. During measurement 4, $15 \mathrm{nM}$ of $R_{2} Q_{2}$ was added, and only red fluorescence was recorded. From this measurement, the signal of the quenched $R_{2} Q_{2}$ control was also measured. Measurement 5 recorded again the baseline of $\mathrm{Cy} 3$ and $\mathrm{Cy} 5$ fluorescence after the reaction finished. Measurement 6 saturated the solution with $20 \mathrm{nM}$ of $I_{\mathrm{hc}}$ to estimate the total amount of $N$ in the solution. After that, $20 \mathrm{nM}$ of $T$ was added to trigger the rest of $R_{2} Q_{2}$ and quantify it in measurement 7. Quenched control was triggered with $20 \mathrm{nM}$ of IT. All concentrations are defined for a reaction volume of $200 \mu \mathrm{L}$. Cy3 fluorescence was pre-eminently used for species quantification due to its higher signal-to-noise ratio and Cy5 fluorescence to follow the actual reaction kinetics. The positive controls contained more than $10 \mathrm{nM}$ of the labelled reporter strands. The negative controls consisted of only the experimental buffer. 


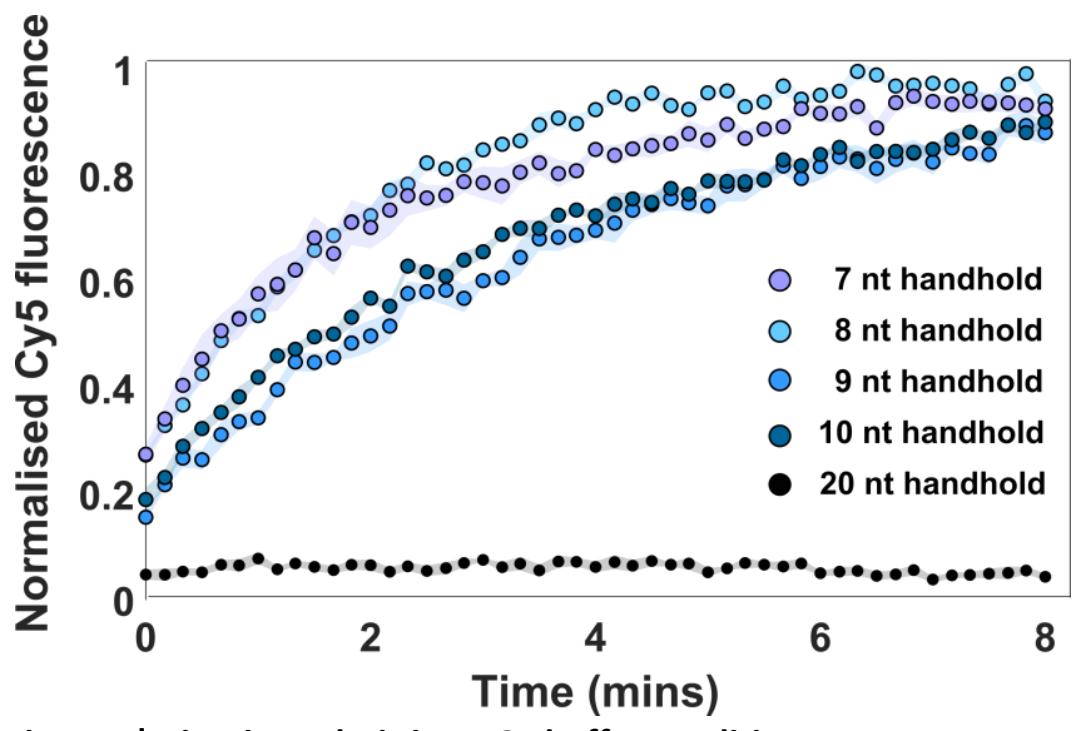

Supplementary Figure 5| Kinetic analysis in PAGE buffer condition.

This control followed the experimental procedure contained in Supplementary Table 3, using a reaction buffer $12.5 \mathrm{mM} \mathrm{MgCl} 2$ in $0.5 \mathrm{XTAE}$. Mean of the normalised fluorescence data of the addition of R2Q2 (Measurement 4) after the reaction of systems with variable handhold lengths, a 2 nt spacer, and a $3 \mathrm{nt}$ toehold that has reacted with $/ \mathrm{hc}$. Each trajectory included its standard error of the mean (shaded) for $n=4$. Fluorescence traces were normalised with the median value obtained in measurement 5 (except the $20 \mathrm{nt}$ handhold condition, which was normalised with measurement 6). As observed in main text Figure 5, the reaction rate is still inversely correlated with handhold length, saturating at $8 \mathrm{nt}$ long. 


\section{Supplementary Note III: Selective production of complexes far-from- equilibrium}

The aim of the experiment was to determine the rate difference between the three competing displacement reactions. To minimise the effect produced by any concentration uncertainty, all $I$ strands and reporter complexes were present at much higher concentrations than the reaction trigger (10 $\mathrm{nM} \mathrm{TN}$ ). Each set of three different experiments was assayed simultaneously using the same aliquots of $I_{x}$ strands and reporter complexes to ensure consistency between the different conditions. At the end of each experiment, all wells were triggered by adding $T$ strand to check that all $I_{x}$ and reporter complexes were present. Steps of the experiments and an example of the fluorescence traces are shown in Supplementary Table 4 and Supplementary Figure 6. Fluorescence signal in this class of experiments was not calibrated as in previous ones because they did not need to be fitted. The gain was set to arbitrary values that do not saturate for $30 \mathrm{nM}$ of each reporter strand. No bleed-through fluorescence was detected between channels during the experiments. The handhold sequences used during the experiments were: the first $8 \mathrm{nt}$ of sequence used during the HMSD characterisations for RepA, a similar sequence with the same GC content for RepB, and the sequence complementary to Iwc for RepC (Supplementary Table 23).

\section{Supplementary Table 4| Detailed experimental procedure to produce far-from-equilibrium complexes}

The three $T N_{x}$ conditions and their controls were triggered by mechanical injection and measured simultaneously. All the tested $T N$ were triggered with a $2 \mathrm{nt}$ proximal toehold and contained an $8 \mathrm{nt}$ handhold and a 2 nt spacer.

\begin{tabular}{|c|c|c|c|c|}
\hline Conditions & Addition & Purpose & $\begin{array}{l}\text { Expected concentrations in } \\
200 \mu \mathrm{L} \\
(\mathrm{nM})\end{array}$ & $\begin{array}{l}\text { Volume } \\
\qquad(\mu L)\end{array}$ \\
\hline Measurement 1 & $\begin{array}{c}20 \mu \mathrm{L} \text { of each Rep }(300 \\
\mathrm{nM})+130 \mu \mathrm{L} \text { Mix of } / \\
(38.46 \mathrm{nM} \text { each) }\end{array}$ & $\begin{array}{l}\text { Estimate residual } \\
\text { fluorescence baseline }\end{array}$ & $\begin{array}{c}\operatorname{Rep}_{A}: 30 ; \operatorname{Rep}_{B}: 30 ; \operatorname{Rep}_{\mathrm{C}}: 30 \\
I_{A}: 25 ; I_{B}: 25 ; I_{C}: 25\end{array}$ & 190 \\
\hline Measurement 2 & $10 \mu \mathrm{L} T N_{x}(200 \mathrm{nM})$ & System kinetics & Evolve with time & 200 \\
\hline Measurement 3 & 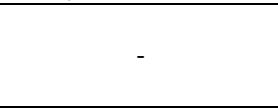 & $\begin{array}{l}\text { Give max. fluorescence } \\
\text { value for each Rep }\end{array}$ & $\begin{array}{l}\text { The I and Rep with the same handhold } \\
\text { complementarity as } N_{x} \text { will have } \\
\text { reacted. }\end{array}$ & 200 \\
\hline Measurement 4 & $20 \mu \mathrm{L} T(1000 \mathrm{nM})$ & $\begin{array}{l}\text { Trigger Rep to test that } \\
\text { all species were present }\end{array}$ & $\begin{array}{l}\text { Rep } x / T: \sim 25 ; \text { Rest of } \operatorname{Rep}_{x} \text { could react } \\
\text { with } T \text { free in solution. }\end{array}$ & 220 \\
\hline
\end{tabular}




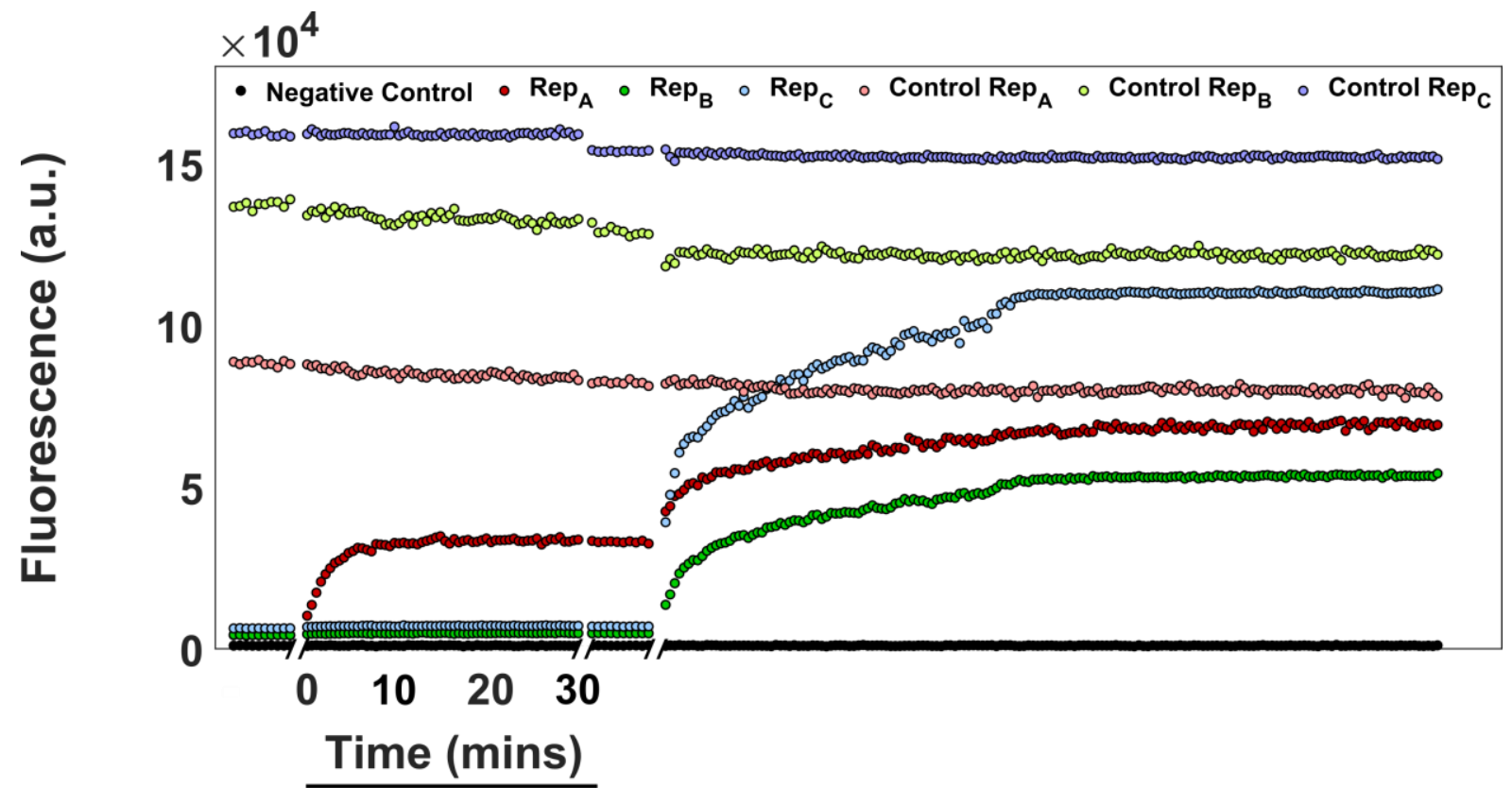

Meas.1 Meas.2 Meas.3 Meas.4

Supplementary Figure 6| Example of raw experimental data from the production of complex $I_{A} T$ The experiment consisted of three competing reaction systems with different $8 \mathrm{nt}$ handholds, $2 \mathrm{nt}$ spacer and $2 \mathrm{nt}$ toehold at $25^{\circ} \mathrm{C}\left(8 / 2 / 2 \_25\right)$. Measurement 1 contains the baseline of the experiment produced by $30 \mathrm{nM}$ of each quenched reporter and $25 \mathrm{nM}$ of each $I_{x}$. Then, $10 \mathrm{nM}$ of $T N_{A}$ were added, and the kinetics recorded in measurement 2 . After the reaction finished, measurement 3 estimated the total amount of produced IT. The fluorescence signal observed for Rep $\mathrm{A}_{\mathrm{A}}$ during this measurement was then used to normalise the Cy5 fluorescence of all the experiments. After the experiment, in measurement 4, the wells were saturated with $20 \mathrm{nM}$ of $T$ to trigger all the reporter complexes. All concentrations are defined for a reaction volume of $200 \mu \mathrm{L}$. The positive controls contained more than $30 \mathrm{nM}$ of the labelled reporter strands. The negative control consisted of only the experimental buffer. 


\section{Supplementary Note IV: Experimental protocol for fluorescence calibration curves}

Two fresh aliquots of $1 \mu \mathrm{M}$ of the fluorophore-labelled reporter strand were created from a stock solution of $100 \mu \mathrm{M}$. The aliquots contained $10 \mu \mathrm{L}$ of the stock solution in $990 \mu \mathrm{L}$ of TAE $1 \mathrm{x}$ buffer $(\mathrm{pH}=8.3)$. From each $1 \mu \mathrm{M}$ aliquot, two more were created with a final concentration of $200 \mu \mathrm{M}$, producing a total of four $200 \mu \mathrm{M}$ aliquots. Four replicas of the calibration curve were pipetted from the $200 \mu \mathrm{M}$ aliquots, for a range of 1 to $15 \mu \mathrm{M}$ and a final volume of $160 \mu \mathrm{L}$. The $200 \mu \mathrm{M}$ aliquot used for each point was chosen randomly. The volume was increased to $200 \mu \mathrm{L}$ with $40 \mu \mathrm{L}$ of a solution of $5 \mathrm{M} \mathrm{NaCl}$ in TAE 1x, for a final experimental buffer of $1 \mathrm{M} \mathrm{NaCl}$ in TAE 1x. The plate was incubated at the assay temperature for 30 minutes until the fluorescence signal stabilized. Each calibration point was obtained from the median value of 10 data points. For each replica of the calibration curve, calibration points were treated as outliers when deviating more than $2 \mathrm{nM}$ from its expected value in a preliminary linear regression.

The photomultiplier gain was set so that its dynamic range was $1.25 x$ the highest fluorescence value in the calibration experiments. Two replicas for the calibration curve of $\mathrm{Cy} 5$ contained concentration values up to $16 \mathrm{nM}$. No bleed-through fluorescence between Cy5 and Cy3 was observed during these calibration experiments. 


\section{Supplementary Note V: Fitting procedure for fluorescence calibration curves}

The value of a negative control consisting of only the experimental buffer was subtracted from all the points in the calibration curves. All calibration curves were fitted with a weighted linear regression to a model of the form $f(x)=a x$. The residuals were weighted with the inverse of the fluorescence reading to account for the Poisson distribution of the photomultiplier counts ${ }^{3}$. Regression coefficient value is reported with $95 \%$ confidence bounds. Fitting results and their residuals are shown in Supplementary Figures 7 to 10.

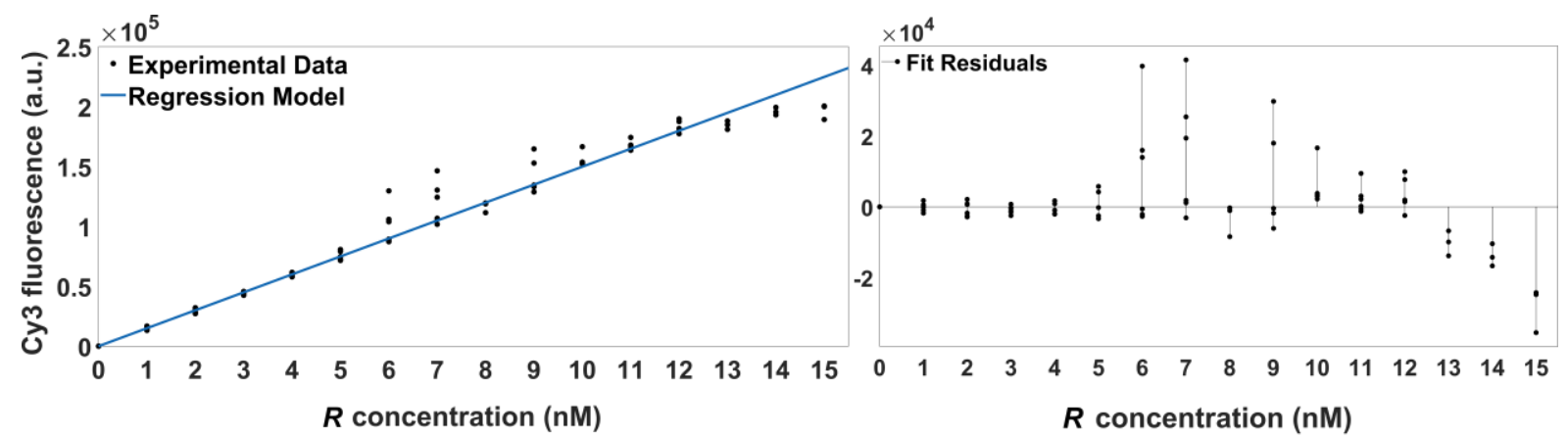

Supplementary Figure $7 \mid \mathrm{RQ}$ reporter complex (Cy3) calibration curve at $25^{\circ} \mathrm{C}$. Gain 2251

Used to extrapolate concentrations for all the characterisation experiments at $25^{\circ} \mathrm{C}$. Left: Regression line. Right: Fit residuals. $a=1.498 \mathrm{e}+04(1.469 \mathrm{e}+04,1.527 \mathrm{e}+04)$. Goodness of fit: SSE: $6.915 \mathrm{e}+04$. Rsquare: 0.9915. Adjusted R-square: 0.9915. RMSE: 27.87.

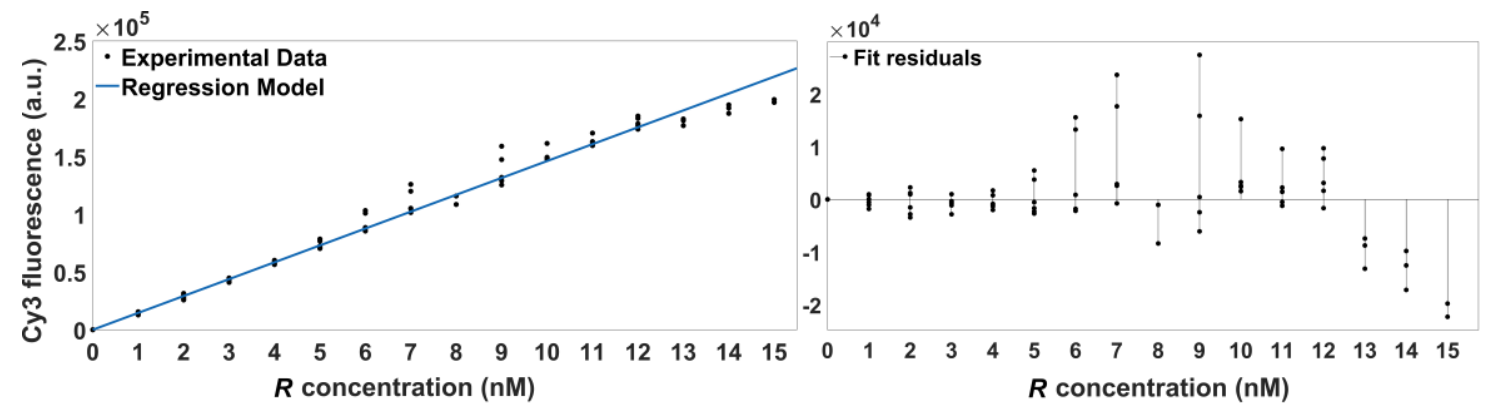

Supplementary Figure $8 \mid \mathrm{RQ}$ reporter complex (Cy3) calibration curve at $37^{\circ} \mathrm{C}$. Gain 2336 Used to extrapolate concentrations for all the characterisation experiments at $37^{\circ} \mathrm{C}$. Left: Regression line. Right: Fit residuals. a=1.461e+04 (1.44e+04, 1.481e+04). Goodness of fit: SSE: $3.386 \mathrm{e}+04$. Rsquare: 0.9955. Adjusted R-square: 0.9955. RMSE: 19.5. 


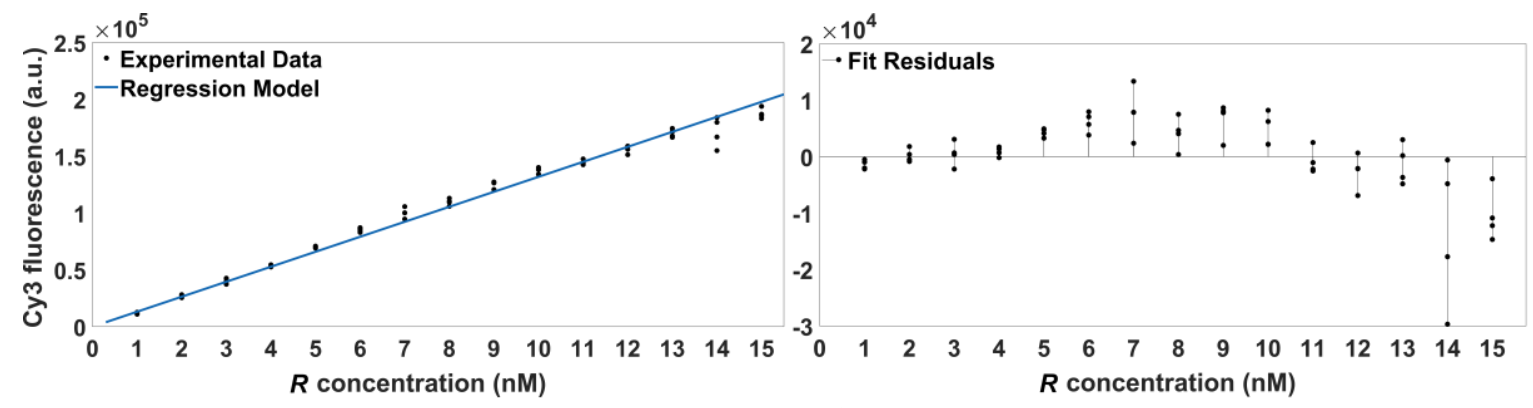

Supplementary Figure 9|RQ reporter complex (Cy3) calibration curve at 25 degrees. Gain 2230

Used to extrapolate concentrations for detachment assays. Gain was reduced during this calibration to avoid the frequency of detector saturation events. Left: Regression line. Right: Fit residuals. $a=$ $1.319 \mathrm{e}+04$ (1.298e+04, 1.339e+04). Goodness of fit: SSE: 2.189e+04. R-square: 0.9923. Adjusted Rsquare: 0.9923. RMSE: 19.26 .

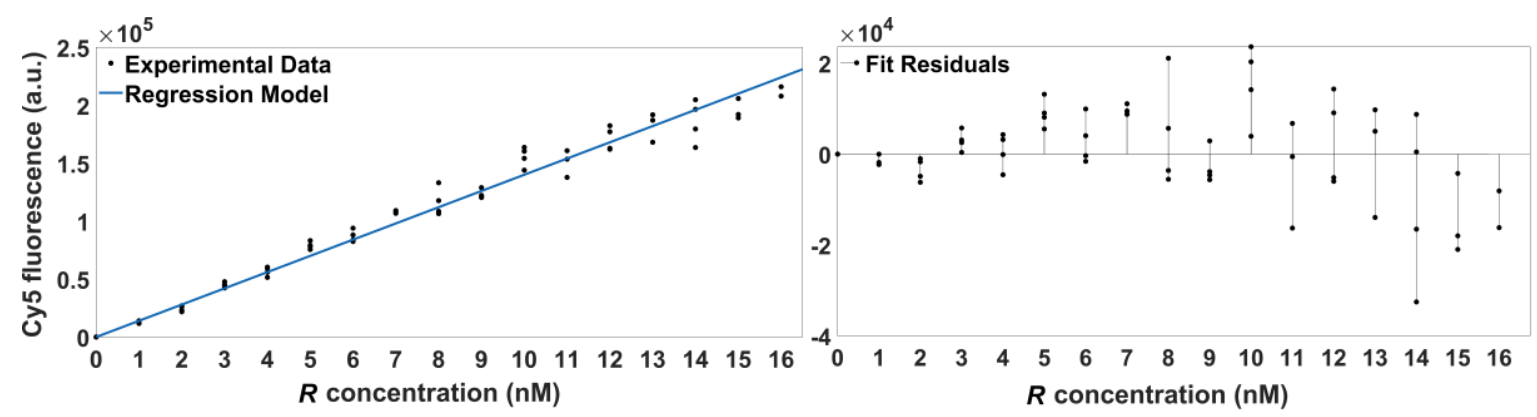

Supplementary Figure 10| $R_{2} Q_{2}$ reporter complex (Cy5) calibration curve at 25 degrees. Gain 2816 Used to extrapolate concentrations for detachment assays. Left: Regression line. Right: Fit residuals. $a=1.4 e+04$ (1.371e+04, 1.43e+04). Goodness of fit: SSE: 4.667e+04. R-square: 0.993. Adjusted Rsquare: 0.993. RMSE: 27.22 . 


\section{Supplementary Note VI: Fluorescence data processing}

To compare calibration lines with the fluorescence signal produced during the experiments, the experimental fluorescence signal was processed as follows:

Step 1. Removing background fluorescence produced by experimental buffer:

The value of the negative control at each time point was subtracted from the fluorescence value of all wells.

Step 2. Merging all measurements in a single file:

All measurements, except the actual kinetics, were converted in a single data point per well, with its fluorescence value given by the median of all the data points in that set. Then, all the single data points corresponding to the same experiment were merged into a file that also contained the complete kinetics of the experiment.

Step 3. Calculation of the fluorescence variation in the positive control signal:

During experiments, the plate could be taken out of the plate reader or liquids at slightly different temperatures could be injected. These temperature fluctuations produced distortions in the fluorescence signal, especially for assays at $37^{\circ} \mathrm{C}$. The fluorescence value of the positive control for each data point was used to track such distortions of the fluorescence signal due to temperature changes. The positive control value during the $1^{\text {st }}$ measurement was the reference value for the experiment since all wells were at the expected experimental temperature, and any photobleaching was assumed to be negligible.

Step 4- Correction of all trajectories with the positive control ratio:

All data points were multiplied by the ratio between the positive control reference value and its value at each time. When more than one positive control of the same species was present in the plate, the positive control ratio at each time was obtained by calculating the mean of the ratio of all positive controls in the plate. This approach could be applied because the variation of fluorescence produced by temperature changes is proportional to the fluorescent signal ${ }^{4}$. Supplementary Figure 11 contains an example of the signal processing result.

\section{Step 4.1- For quick measurements (Reporter Characterisation kinetics):}

The correction described in step 4 was detrimental to the precision of the measurements when positive control fluorescence traces had a lower signal-to-noise ratio, and signal fluorescence artefacts were more common. This happened particularly during quick measurements, like $R Q$ reporter characterisations. Besides, since all the kinetics were measured individually, positive controls could not be measured in parallel. For these cases, all the data points in the kinetics section were just multiplied by the ratio between the reference control value and control value obtained in mearement 4, after the kinetics finished (See Supplementary Note I).

\section{Step 5- Correction for residual fluorescence:}

A difference between the signal of the calibration curves and the experiments is that in the former, no quenched $R Q$ is present. To compare both fluorescence signals to infer $R Q$ concentrations, the background due to the residual fluorescence of quenched $R Q$ complexes needed to be subtracted from the experimental measurements. The residual fluorescence was subtracted from the signal $F_{i}$ at data point $i$ using the formula:

$$
F_{i}^{*}=F_{\max } \frac{F_{i}-F_{1}}{\left(F_{\max }-F_{1}\right)}
$$


Here, $F_{i}^{*}$ is the data point $i$ with the corrected background fluorescence, $F_{1}$ the residual fluorescence background value during measurement 1 and $F_{\max }$ the final fluorescence value in measurement 6 (Supplementary Note I). The method assumes that any difference of fluorescence with the negative control during measurement 1 is due to unquenched $R Q$, being that increment proportional to $[R Q]_{0}$ (Supplementary Note I). The fluorescence was corrected in such a way that $F_{1}$ became 0 and $F_{\max }$ did not change.

Step 6- Transformation of fluorescence arbitrary units to nM:

Once all the corrections are applied, all the values of the data points are transformed to $\mathrm{nM}$ by dividing them by the slope of their corresponding calibration curve (Supplementary Note V).
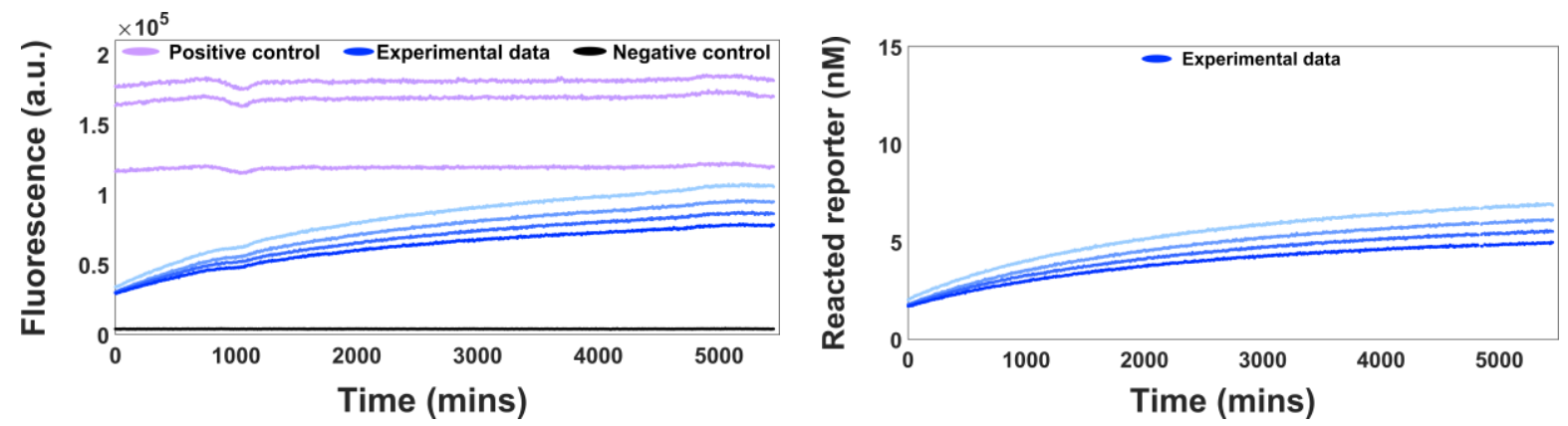

\section{Supplementary Figure 11 | Example of fluorescence data processing}

Four traces from a reaction system with a $20 \mathrm{nt}$ handhold, $5 \mathrm{nt}$ spacer and $2 \mathrm{nt}$ proximal toehold (20/5/2_25), triggered with [4-7] nM of $I_{w c}$. Left) Raw data. Irregularities during the experiment can produce bumps in the fluorescence that tampers the fitting process. These fluctuations appeared in all trajectories and controls contained in the plate. This example contained three different positive controls for Cy3. Even when controls did not have the same Cy3 concentrations, their variations in fluorescence were proportional. Right) The same data after applying the fluorescence processing pipeline. Fluorescence units were transformed to $\mathrm{nM}$, and the bumps disappeared. All concentrations are defined for a reaction volume of $200 \mu \mathrm{L}$. 


\section{Supplementary Note VII:Additional fluorescence processing}

Out of range fluorescence:

The photomultiplier gain was set so that its dynamic range was $1.25 x$ the fluorescence signal produced by $15 \mathrm{nM}$ of Cy3. However, during some assays, the total amount of $R Q$ was slightly over that concentration. This resulted in a fluorescence signal over the photomultiplier detection limit when measuring the fluorescence produced by $[R Q]_{0}$. To correct these out-of-range data points, the gain was slightly decreased until no saturation events appeared. After that, the fluorescence values from the saturated and the low-gain measurement were compared. The fluorescence change ratio between the measurements was calculated with the positive controls and the samples that did not saturate. The corrected fluorescence values of the saturated data points were estimated by multiplying the data point at a low gain by the mean of the obtained ratios. This correction assumed that linearity is maintained at the two different gains. Any error introduced in the inferred $[R Q]_{0}$ using this approximation is considered negligible since variations of $[R Q]_{0}$ had a small impact in the fitting procedure for most of the systems. All files modified this way are listed in the file Original_files/Raw_files_modifications.txt found in www.zenodo.org/record/4389212.

Out of range fluorescence during kinetics:

When out-of-range outliers appeared during the kinetics, that sample was discarded. For experiments with kinetics longer than 2 hours, the datapoint with the outlier was removed from all samples instead.

Estimation of residual fluorescence during kinetic analysis of handhold detachment:

During detachment assays $R_{2} Q_{2}$ was used to trigger the reaction. This fact precluded the measurement of the Cy5 fluorescence baseline of each fluorescence trace. Thus, Cy 5 fluorescence baselines were calculated by using the quenched control of each experiment. The quenching efficiency for $R_{2} Q_{2}$ was estimated from the quenched control by calculating the ratio between its initial fluorescence value and its fluorescence value once fully triggered. The quenching efficiency was multiplied by the maximum fluorescence of each trace to calculate its Cy5 fluorescence baseline. The obtained Cy5 baselines were used to correct the fluorescence traces with Supplementary equation 1 , in the same way as with $R Q$. 


\section{Supplementary Note VIII: $\quad$ Reaction controls for $R Q$}

The control aim was to prove that no leak reaction occurred between $T N$ and $R Q$. A plate with 18 reactions was set. The 18 wells contained a solution of the $R Q$ reporter and $T N$ with a $7 \mathrm{nt}$ toehold. 6 different systems were tested in triplicate: 0,5 and $20 \mathrm{nt}$ long handholds with spacers of 0 and $8 \mathrm{nt}$. The solution of $R Q$ and $T N$ was incubated for more than 24 hours without producing any fluorescent signal. After that, three different concentrations of $I_{h c}(4,6$ and $8 \mathrm{nM})$ were added to each system to prove the proportionality between the fluorescence signal and $\left[I_{h c}\right]_{0}$. Steps of the reaction control for $R Q$ and the obtained results are shown in Supplementary Table 5 and Supplementary Figure 12. An additional control was assayed to test if the reporter could not be triggered by handhold complementarity alone. The modification included to the additional control was that the added Istrand only had complementarity with the handhold domain in $N$ and not with $T$ (Invader-random 3', See Supplementary Table 22). Fluorescence was measured for more than 30 hours without any increase of signal observed. Steps of the control using / without $T$ complementarity and the obtained results are shown in Supplementary Table 6 and Supplementary Figure 13.

\section{Supplementary Table 5 | Steps of the reaction control for $R Q$}

All the trajectories in the plate and their controls were triggered by mechanical injection and measured simultaneously. All the tested $T N$ contained a $7 \mathrm{nt}$ proximal toehold.

\begin{tabular}{|c|c|c|c|c|}
\hline Steps & Addition & Description & Purpose & $\begin{array}{c}\text { Volume } \\
(\boldsymbol{\mu l})\end{array}$ \\
\hline Measurement 1 & $\begin{array}{c}10 \mu \mathrm{L} R Q(300 \mathrm{nM})+ \\
10 \mu \mathrm{L} T N(200 \mathrm{nM})+ \\
130 \mu \mathrm{L} \mathrm{Buffer}\end{array}$ & $\begin{array}{c}\text { Kinetics during } 16.5 \text { hours } \\
\text { of the } R Q \text { basal } \\
\text { fluorescence }\end{array}$ & $\begin{array}{c}\text { Prove that the presence of } T N \text { does not } \\
\text { trigger } R Q\end{array}$ & 150 \\
\hline Measurement 2 & - & $\begin{array}{c}\text { Measurement after } \\
\sim 24 \text { hours }\end{array}$ & Prove signal has not changed & 150 \\
\hline Measurement 3 & $\begin{array}{c}{[40 / 30 / 20] \mu \mathrm{L} / \mathrm{hc}(40 \mathrm{nM})+} \\
{[10 / 20 / 30] \mu \mathrm{L} \mathrm{Buffer}}\end{array}$ & Trigger TN & $\begin{array}{c}\text { Prove that / can trigger the reaction, and } \\
\text { check proportionality of fluorescence } \\
\text { signal. }\end{array}$ & 200 \\
\hline Measurement 4 & $10 \mu \mathrm{L} /(400 \mathrm{nM})$ & Trigger all TN & Prove triggering of total $T N$ & 210 \\
\hline
\end{tabular}

\section{Supplementary Table 6| Steps of the control for I without T-complementarity}

All the trajectories in the plate and their controls were triggered by mechanical injection and measured simultaneously. All the tested $T N$ contained a $7 \mathrm{nt}$ proximal toehold.

\begin{tabular}{|c|c|c|c|c|}
\hline Steps & Addition & Description & $\begin{array}{c}\text { Polume } \\
(\boldsymbol{\mu l})\end{array}$ \\
\hline Measurement 1 & $\begin{array}{c}10 \mu \mathrm{L} R Q(300 \mathrm{nM})+ \\
130 \mu \mathrm{L} \mathrm{Buffer}\end{array}$ & Measure baseline & Remove the residual fluorescence & 140 \\
\hline Measurement 2 & $10 \mu \mathrm{LN}(200 \mathrm{nM})$ & Measure new baseline & Check the signal produced by $[N]_{\text {excess }}$ & 150 \\
\hline Measurement 3 & $50 \mu \mathrm{L} /(40 \mathrm{nM})$ & $\begin{array}{c}\text { Add } / \text { without } T \\
\text { complementarity. }\end{array}$ & $\begin{array}{c}\text { Prove that handhold interaction does } \\
\text { not trigger the reporter. }\end{array}$ \\
\hline Measurement 4 & - & Measure after one day & Prove signal has not changed & 200 \\
\hline
\end{tabular}



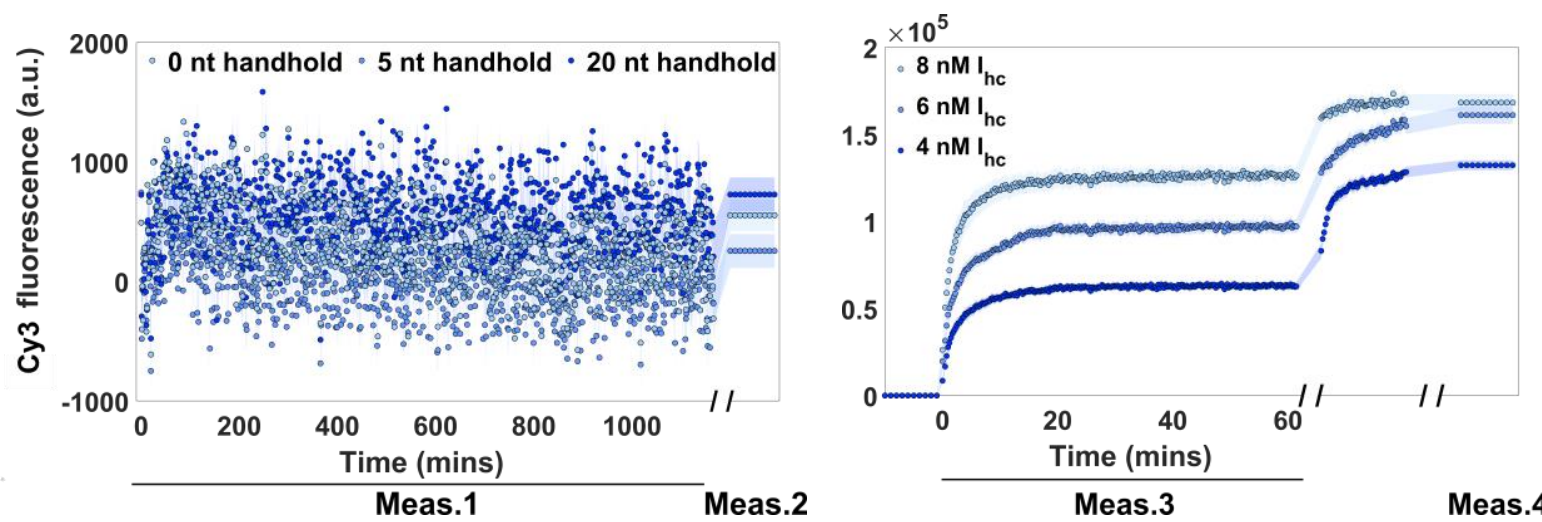

Supplementary Figure 12| Reporter $R Q$ control, proving the absence of leak when mixed with unreacted TN

Processed fluorescence data of 6 different systems tested in triplicate: 0,5 and $20 \mathrm{nt}$ long handholds with spacers of 0 and $8 \mathrm{nt}$, and a proximal toehold of $7 \mathrm{nt}$. Mean of the signal produced for each handhold length with its standard error (shaded). The baseline of fluorescence produced by $[N]_{\text {excess }}$ was removed to clearly show the scale of the fluorescence signal noise. Left) After the kinetics of measurement 1 , measurement 2 is taken after approximately one day. No significant increase in the fluorescence signal could be observed. Right) For each replica of each system, a different concentration of $I_{h c}$ was added: 8,6 and $4 \mathrm{nM}$. The produced signal is proportional to the concentration of $I_{h c}$, which allows the quantification of the strand. All concentrations are defined for a reaction volume of $200 \mu \mathrm{L}$.

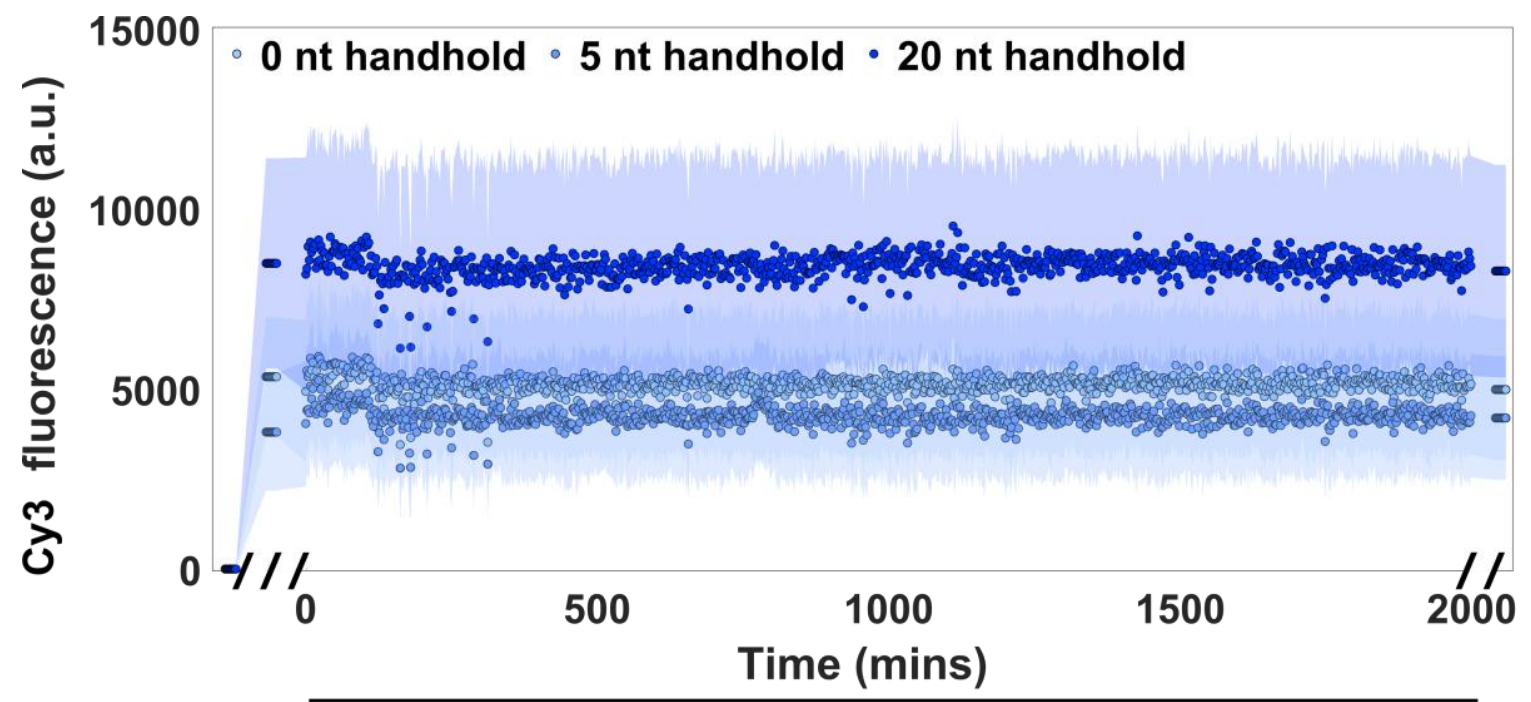

Meas.1//2

Meas.3

Meas.4

Supplementary Figure 13| Reporter $R Q$ control using I without $T$-complementary, proving the absence of reaction when using an I strand that is only handhold complementary

Processed fluorescence data of 6 different systems tested in triplicate: 0,5 and $20 \mathrm{nt}$ long handholds with spacers of 0 and $8 \mathrm{nt}$, and a proximal toehold of $7 \mathrm{nt}$. Mean of the signal produced for each handhold length with its standard error (shaded) for $n=9$. Measurement 1 set the baseline produced by $15 \mathrm{nM}$ of quenched $R Q$. Measurement 2 set a new baseline produced by the $[N]_{\text {excess }}$ after adding $10 \mathrm{nM} T N$. In measurement $3,10 \mathrm{nM}$ of I without $T$ complementarity is added and measured for at least 30 hours. No increase in the fluorescence signal was observed. Measurement 4 is taken after approximately one day, and no increase is observed either. All concentrations are defined for a reaction volume of $200 \mu \mathrm{L}$. 


\section{Supplementary Note IX: Reaction controls for $\boldsymbol{R}_{\mathbf{2}} \boldsymbol{Q}_{\mathbf{2}}$}

Five different conditions were assayed in duplicate, testing different qualities of $R_{2} Q_{2}$. The used $T$ contained a $3 \mathrm{nt}$ proximal toehold and, to trigger the reporter $R_{2} Q_{2}$, an extra $5^{\prime}$ domain. Condition 1 proved that the reporter could be quickly triggered by the addition of the complex IT. During the experiments of condition $2, R_{2} Q_{2}$ was quickly triggered when a $T N$ with a short handhold (6 nt) reacted with $I_{h c}$. Conditions 3 and 4 showed that the leak reaction of $R_{2} Q_{2}$ with $T N$ (reported in Supplementary Note II) is independent of handhold length. Besides, condition 4 proved that systems with $20 \mathrm{nt}$ handholds could not trigger $R_{2} Q_{2}$ quickly after displacement since the produced IT cannot detach from the handhold. Finally, condition 5 showed that $R_{2} Q_{2}$ does not change its fluorescence signal for 3 hours in the presence of only $I_{h c}$. Concentrations of $R_{2} Q_{2}$ differed between conditions due to an unoptimized annealing process of $R_{2} Q_{2}$. However, the qualitative effect of each condition can still be observed. Steps for each condition and the obtained results are shown in Supplementary Table $\mathbf{7}$ and Supplementary Figure 14.

\section{Supplementary Table 7 | Steps of the $R_{2} Q_{2}$ control}

All the trajectories in the plate and their controls were triggered by mechanical injection and measured simultaneously. All the tested $T N$ contained a 3 nt proximal toehold. Each column of the table contains the species added to the conditions before each measurement.

\begin{tabular}{|c|c|c|c|c|}
\hline Conditions & Measurement 1 & Measurement2 & Measurement 3 & Measurement 4 \\
\hline Condition 1 & $\begin{array}{c}75 \mu \mathrm{L} R_{2} Q_{2}(40 \mathrm{nM})+ \\
75 \mu \mathrm{L} \text { Buffer }\end{array}$ & $\begin{array}{c}10 \mu \mathrm{L} I T(200 \mathrm{nM})+40 \\
\mu \mathrm{L} \text { Buffer }\end{array}$ & $10 \mu \mathrm{L} / \mathrm{hc}(400 \mathrm{nM})$ & $10 \mu \mathrm{L} / T(400 \mathrm{nM})$ \\
\hline Condition 2 & $\begin{array}{c}75 \mu \mathrm{L} R_{2} Q_{2}(40 \mathrm{nM})+ \\
+75 \mu \mathrm{L} \text { Buffer }\end{array}$ & $\begin{array}{c}10 \mu \mathrm{L} T N(6 / 2 / 3)(200 \mathrm{nM} \\
++40 \mu \mathrm{L} / \mathrm{hc}(40 \mathrm{nM})\end{array}$ & $10 \mu \mathrm{L} / \mathrm{hc}(400 \mathrm{nM})$ & $10 \mu \mathrm{L} / T(400 \mathrm{nM})$ \\
\hline Condition 3 & $\begin{array}{c}75 \mu \mathrm{L} R_{2} Q_{2}(40 \mathrm{nM})+ \\
+75 \mu \mathrm{L} \text { Buffer }\end{array}$ & $\begin{array}{c}10 \mu \mathrm{L} T N(0 / 2 / 3)(200 \mathrm{nM} \\
++40 \mu \mathrm{L} / \mathrm{hc}(40 \mathrm{nM})\end{array}$ & $10 \mu \mathrm{L} / \mathrm{hc}(400 \mathrm{nM})$ & $10 \mu \mathrm{L} / T(400 \mathrm{nM})$ \\
\hline Condition 4 & $\begin{array}{c}75 \mu \mathrm{L} R_{2} Q_{2}(40 \mathrm{nM})+ \\
+75 \mu \mathrm{L} \text { Buffer }\end{array}$ & $\begin{array}{c}10 \mu \mathrm{L} T N(20 / 2 / 3)(200 \\
\mathrm{nM})+40 \mu \mathrm{L} / \mathrm{hc}(40 \mathrm{nM})\end{array}$ & $10 \mu \mathrm{L} / \mathrm{hc}(400 \mathrm{nM})$ & $10 \mu \mathrm{L} / T(400 \mathrm{nM})$ \\
\hline Condition 5 & $\begin{array}{c}75 \mu \mathrm{L} R_{2} Q_{2}(40 \mathrm{nM})+ \\
+75 \mu \mathrm{L} \text { Buffer }\end{array}$ & $50 \mu \mathrm{L} / \mathrm{hc}(40 \mathrm{nM})$ & $10 \mu \mathrm{L} / \mathrm{hc}(400 \mathrm{nM})$ & $10 \mu \mathrm{L}$ Buffer \\
\hline
\end{tabular}




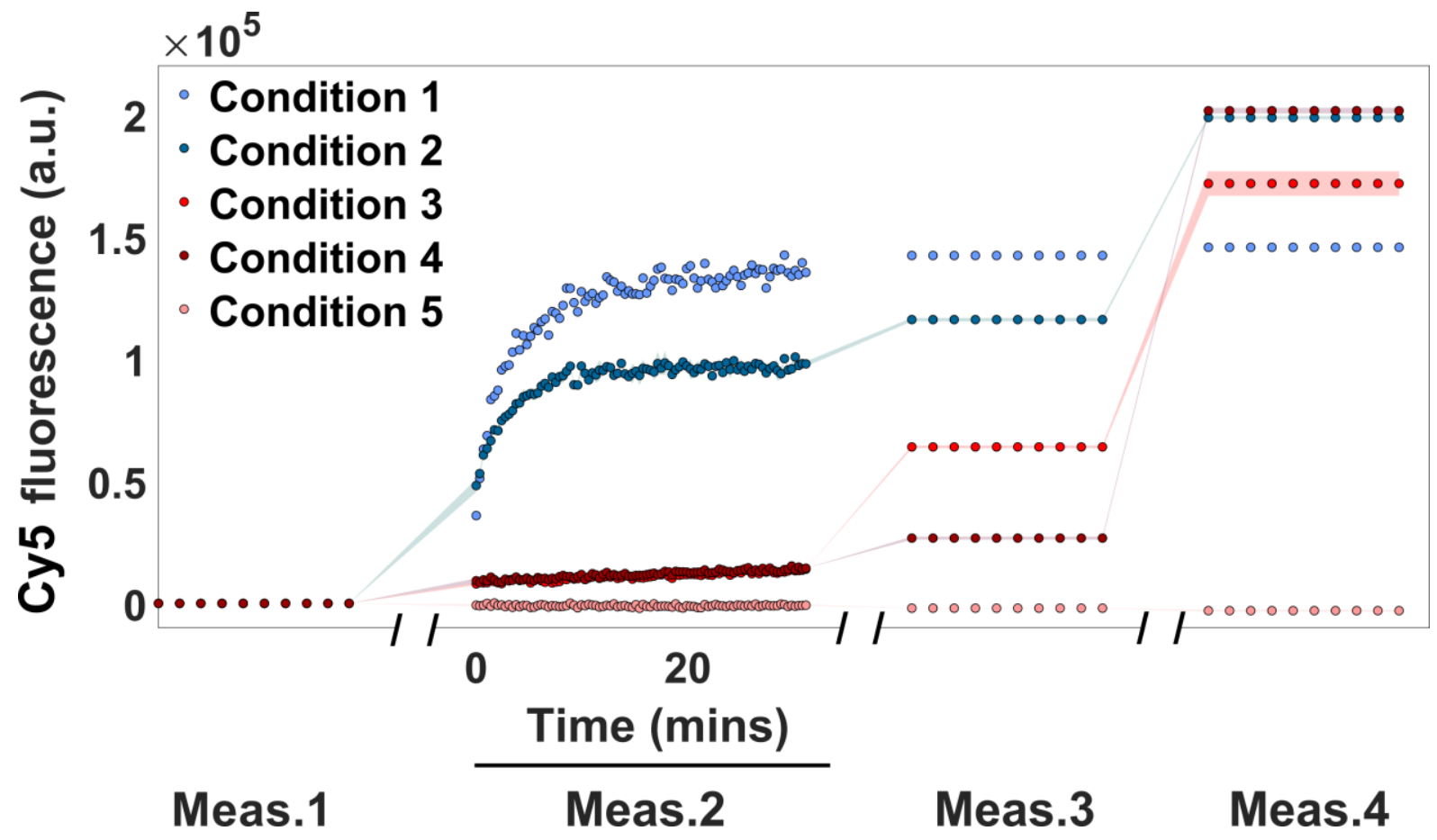

Supplementary Figure $14 \mid$ Reporter $\boldsymbol{R}_{\mathbf{2}} \mathbf{Q}_{\mathbf{2}}$ controls

Processed fluorescence data of five different conditions tested in duplicate (except condition 1). Mean of the signal produced for each condition with its standard error (shaded). The purpose of each condition is described in Supplementary Note VIII. Measure 1 set the baseline produced by $15 \mathrm{nM}$ of quenched $R_{2} Q_{2}$ and measure 2 contains the kinetics of every condition (Described in Supplementary Table 7). Measurement 3 was taken after adding $20 \mathrm{nM} I_{h c}$ to all conditions. During measurement 3 , condition 3 did not reach saturation due to the slow reaction rate of the $3 \mathrm{nt}$ proximal toehold reaction. In the case of condition 4, it did not reach saturation because the produced IT could not detach from the $20 \mathrm{nt}$ handhold and trigger the reporter. Measurement 4 was taken after adding $20 \mathrm{nM} I T$ to all conditions, except condition 5 . All concentrations are defined for a reaction volume of $200 \mu \mathrm{L}$. 


\section{Supplementary Note X: Specificity controls for $\operatorname{Rep}_{A}, \operatorname{Rep}_{B}$ and $\operatorname{Rep}_{C}$}

For the selective production of complexes far-from-equilibrium, not only must the handhold do its part, but the reporters that monitor the reactions $\left(\operatorname{Rep}_{\mathrm{A}}, \operatorname{Rep}_{\mathrm{B}}\right.$, and $\left.\operatorname{Rep}_{\mathrm{C}}\right)$ must be able to discern between the $I T_{X}$ complexes. The purpose of these controls was to confirm that the designed reporters react only with the $I T_{x}$ with which they had handhold complementarity.

Since the design of the reporters was the same as $R_{2} Q_{2}\left(\operatorname{Rep}_{\mathrm{A}}\right.$ being the same reporter) a slow leak reaction was expected in the presence of strand $T$. This leak was slower for $\operatorname{Rep}_{B}$ and $R_{C} p_{C}$, since these reporters contained a non-T-complementary $2 \mathrm{nt}$ clamp at the end of the displaced domain that helped to maintain quencher and fluorescence strands hybridised. For Rep $\mathrm{P}_{\mathrm{A}}$ this clamp was not needed since the additional unspecific interaction between $\mathrm{Cy} 5$ and the quencher was strong enough to stabilize the hybridisation of $\operatorname{Rep}_{\mathrm{A}}$ strands $^{5}$.

The controls tested the rate at which the specific reporters reacted with each $T N$ complex and if any leak could be produced by the interaction of the $I_{x}$ strands alone with the reporter. The results prove that during the time that took the reporters to consume $10 \mathrm{nM}$ of complementary $T N_{x}$, the amount of non-complementary $T N_{x}$ consumed is less than $10 \%$. The additions during each step of the control experiments are described in Supplementary Table 8. Results for $\operatorname{Rep}_{A}, \operatorname{Rep}_{B}$ and $\operatorname{Rep}_{C}$ are shown in Supplementary Figures 15, 16 and 17, respectively.

\section{Supplementary Table 8| Steps of the selectivity controls for Rep complexes}

All the trajectories in the plate and their controls were triggered by mechanical injection and measured simultaneously. The used $T$ contained a $3 \mathrm{nt}$ proximal toehold and an extra $5^{\prime}$ domain to trigger the reporter $R_{2} Q_{2}$. Each column of the table contains the species added to the conditions before each measurement.

\begin{tabular}{|c|c|c|c|c|}
\hline Conditions & Measurement 1 & Measurement 2 & Measurement 3 & Measurement 4 \\
\hline $\boldsymbol{I}_{\boldsymbol{X}}+\boldsymbol{T N}_{\boldsymbol{x}}$ & $\begin{array}{c}20 \mu \mathrm{L} \operatorname{Rep}(300 \mathrm{nM})+ \\
5 \mu \mathrm{L} I_{x}(1 \mu \mathrm{M})+165 \mu \mathrm{L} \text { Buffer }\end{array}$ & $10 \mu \mathrm{L} T N_{x}(200 \mathrm{nM})$ & $20 \mu \mathrm{L} T(1 \mu \mathrm{M})$ & $\begin{array}{c}5 \mu \mathrm{L} \text {-complementary } \\
\text { to the reporter }(1 \mu \mathrm{M})\end{array}$ \\
\hline $\boldsymbol{I}_{\boldsymbol{A}}+\boldsymbol{I}_{\boldsymbol{B}}+\boldsymbol{I}_{\boldsymbol{C}}$ & $\begin{array}{c}20 \mu \mathrm{L} \operatorname{Rep}(300 \mathrm{nM})+5 \mu \mathrm{L} I_{A} \\
(1 \mu \mathrm{M})+5 \mu \mathrm{L} I_{B}(1 \mu \mathrm{M})+ \\
5 \mu \mathrm{L} I_{C}(1 \mu \mathrm{M})+155 \mu \mathrm{L} \text { Buffer }\end{array}$ & $10 \mu \mathrm{L}$ Buffer & $20 \mu \mathrm{L} T(1 \mu \mathrm{M})$ & $\begin{array}{c}5 \mu \mathrm{L} \text {-complementary } \\
\text { to the reporter }(1 \mu \mathrm{M})\end{array}$ \\
\hline
\end{tabular}




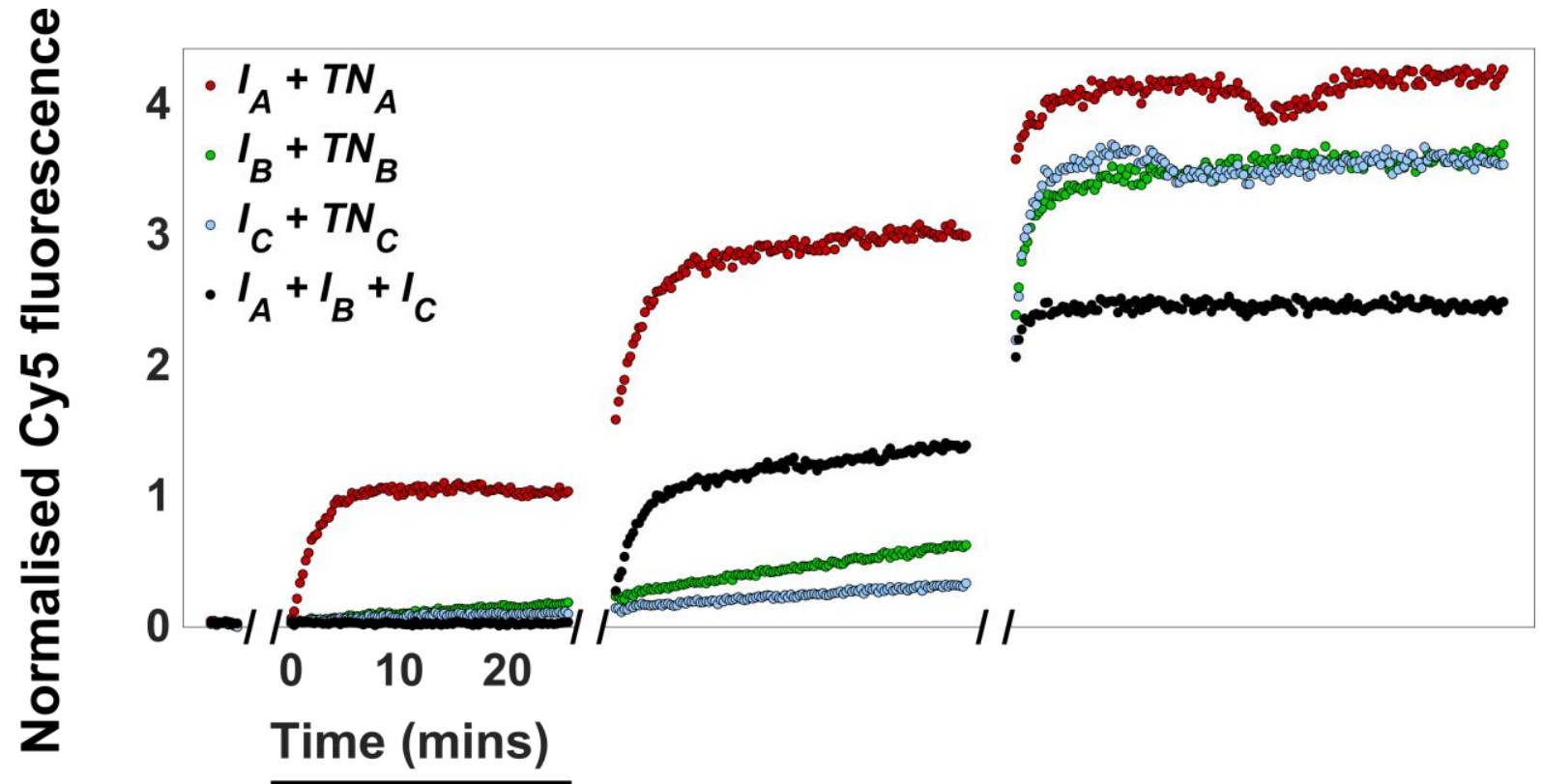

\section{Meas.1 Meas.2 Meas.3 Meas.4}

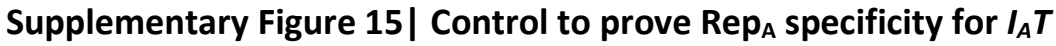

Processed fluorescence data normalised to the max value of $I_{A}+T N_{A}$ condition during measurement 2. Measurement 1 set the baseline produced by $30 \mathrm{nM}$ of quenched $R_{\text {ep }}$ and $25 \mathrm{nM}$ of $I_{x}(75 \mathrm{nM}$ in total in condition $\left.I_{A}+I_{B}+I_{C}\right)$. Measurement 2 contains the kinetics of every condition after adding $10 \mathrm{nM}$ of the specified $T N_{x}$ or just buffer for condition $I_{A}+I_{B}+I_{C}$. Condition $I_{A}+T N_{A}$ is the only one capable of reacting at high speed. Measurement 3 was taken after saturating each condition with $100 \mathrm{nM}$ of $T$, so only the wells containing $I_{A}$ managed to produce fluorescence at an increased rate. In measurement 4 , all wells were saturated with $40 \mathrm{nM}$ of $I_{A}$ to trigger all $\operatorname{Rep}_{A}$. Rep $\mathrm{P}_{A}$, in comparison with the other two reporters, had more leakiness, especially with $T N_{B}$ since handhold $A$ and $B$ have a similar sequence. However, in competition with the other two reporters, the leakiness was greatly reduced. All concentrations are defined for a reaction volume of $200 \mu \mathrm{L}$. 


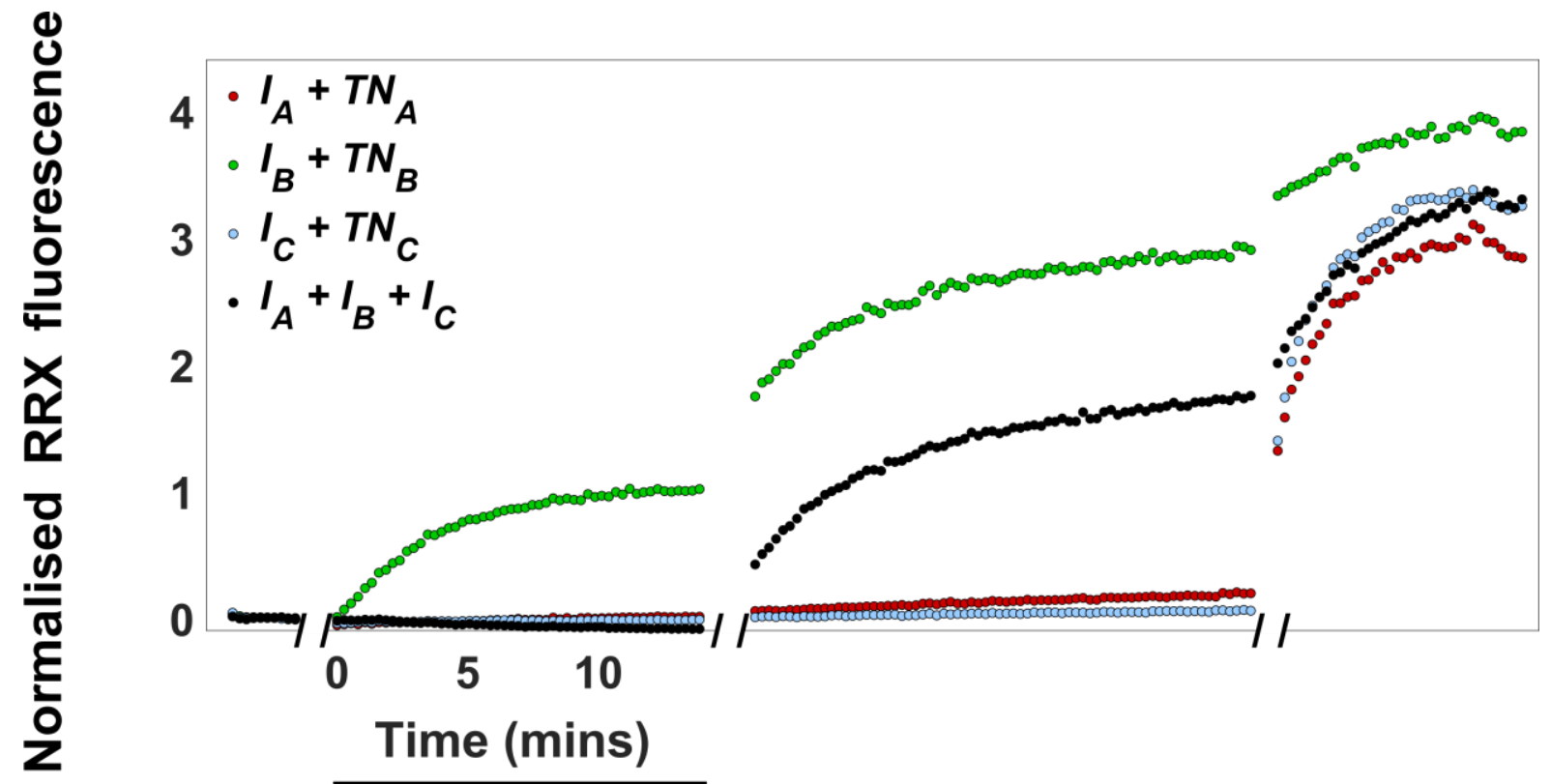

\section{Meas.1 Meas.2 Meas.3}

Meas.4

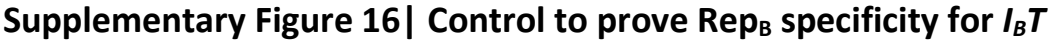

Processed fluorescence data normalised to the max value of $I_{B}+T N_{B}$ condition during measurement 2 . Measurement 1 set the baseline produced by $30 \mathrm{nM}$ of quenched $\operatorname{Rep}_{B}$ and $25 \mathrm{nM}$ of $I_{X}(75 \mathrm{nM}$ in total in condition $\left.I_{A}+I_{B}+I_{C}\right)$. Measurement 2 contains the kinetics of every condition after adding $10 \mathrm{nM}$ of the specified $T N_{x}$ or just buffer for condition $I_{A}+I_{B}+I_{C}$. Condition $I_{B}+T N_{B}$ is the only one capable of reacting at high speed. Measurement 3 was taken after saturating each condition with $100 \mathrm{nM}$ of $T$, so only the wells containing $I_{B}$ managed to produce fluorescence at an increased rate. In measurement 4, all wells were saturated with $40 \mathrm{nM}$ of $I_{B}$ to trigger all $\operatorname{Rep}_{B}$. Rep $p_{B}$ showed less leakiness than $\operatorname{Rep}_{A}$, but the similarity between handholds $A$ and $B$ still resulted in a quicker leak reaction for $I_{A}$ than for $I_{C}$. All concentrations are defined for a reaction volume of $200 \mu \mathrm{L}$. 


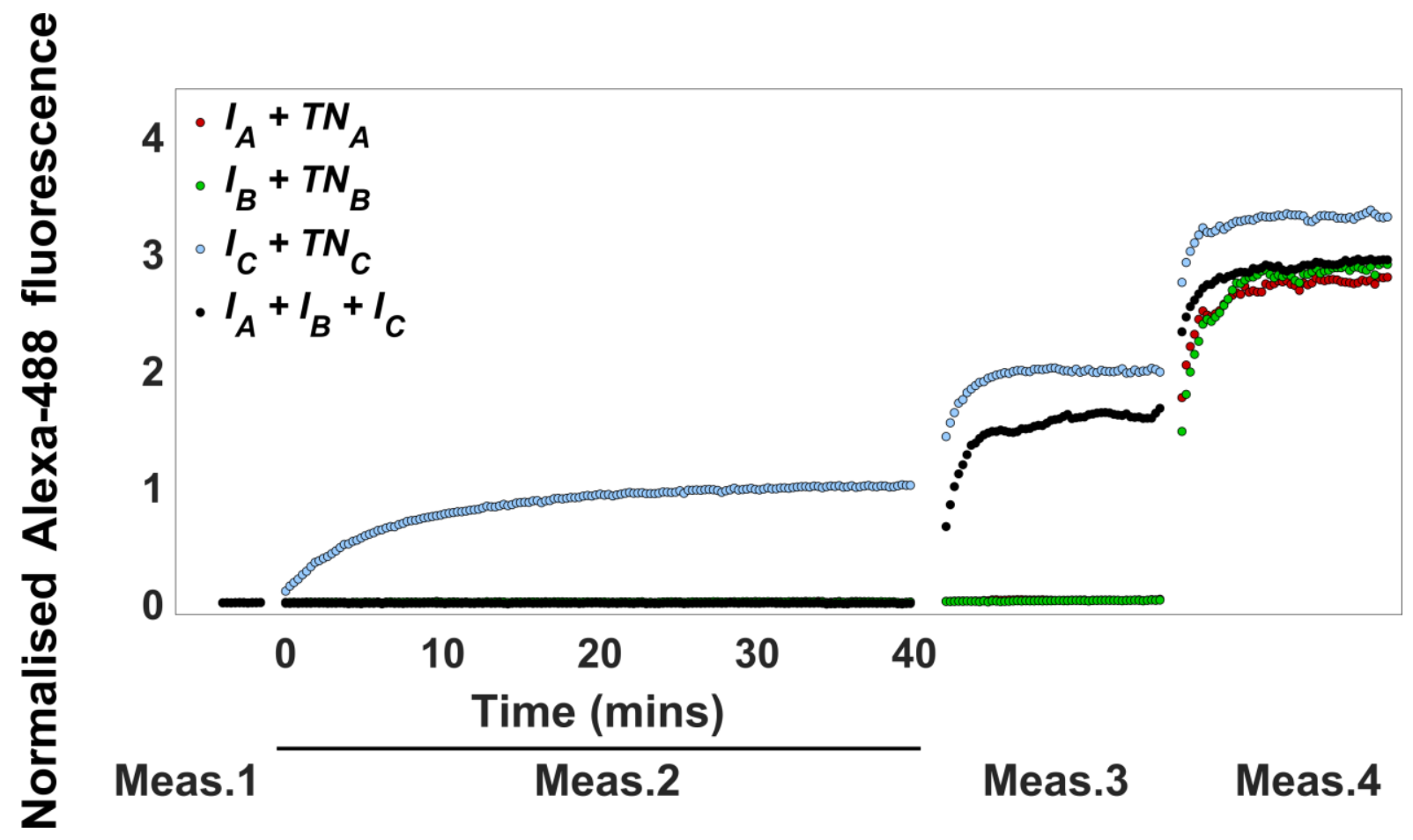

Supplementary Figure 17| Control to prove Repc specificity for $I_{c} T$

Processed fluorescence data normalised to the max value of $I_{C}+T N_{C}$ condition during measurement 2. Measurement 1 set the baseline produced by $30 \mathrm{nM}$ of quenched Rep $\mathrm{p}_{\mathrm{c}}$ and $25 \mathrm{nM}$ of $I_{x}(75 \mathrm{nM}$ in total in condition $\left.I_{A}+I_{B}+I_{C}\right)$. Measurement 2 contains the kinetics of every condition after adding $10 \mathrm{nM}$ of the specified $T N_{x}$ or just buffer for condition $I_{A}+I_{B}+I_{C}$. Condition $I_{C}+T N_{C}$ is the only one capable of reacting at high speed. Measurement 3 was taken after saturating each condition with $100 \mathrm{nM}$ of $T$, so only the wells containing $I_{c}$ managed to produce fluorescence at an increased rate. In measurement 4 , all wells were saturated with $40 \mathrm{nM}$ of $I_{c}$ to trigger all Rep. Rep $p_{c}$ showed no detectable leakiness. All concentrations are defined for a reaction volume of $200 \mu \mathrm{L}$. 


\section{Supplementary Note XI: Implementation of handhold-mediated strand displacement reaction network as an ODE system}

The handhold-mediated strand displacement chemical reaction network is composed of the following reactions:

Binding of / to $T N$ :

N displacement by l:

$$
T N+I \stackrel{k_{b}}{\rightleftharpoons} T N / I .
$$

$$
k_{m}
$$$$
T N / I \rightarrow N / I T \text {. }
$$

Competing TMSD:

$$
\stackrel{k_{t}}{T N+I \rightarrow N+I T .(4)}
$$

Blocking and unblocking of $T N^{\prime}$ s handhold by free in solution IT:

TN with blocked handhold reacting via TMSD:

$$
\begin{gathered}
k_{b} \\
T N+I T \rightleftharpoons T N / I T \text {. (5) } \\
k_{d}
\end{gathered}
$$

$$
T N / I T+I \rightarrow I T+N / I T \text {. }
$$

$\underline{R Q \text { triggering by TMSD. }}$

$$
N / I T+R Q \stackrel{k_{r}}{\rightarrow} R N / I T+Q
$$

Produced quencher strand $(Q)$ is inert.

Free in solution $I T$ attaching and detaching from the handhold in $R N$

$$
R N+I T \stackrel{I T}{\rightleftharpoons} R N / I T \text {. (8) }
$$

Free-in-solution / attaching and detaching from the handhold in $R N$

$$
\begin{gathered}
k_{b} \\
R N+\stackrel{l}{\rightleftharpoons} R N / I . \\
k_{d}
\end{gathered}
$$

$\underline{R N / I \text { triggering } T N \text { and } T N / I T \text { by toehold interaction. }}$

$$
\begin{gathered}
k_{t} \\
T N+R N / I \stackrel{\rightarrow}{\rightarrow}+R N / I T,(10) \\
k_{t} \\
T N / I T+R N / I \rightarrow R N / I T+N / I T .
\end{gathered}
$$

The ODE model did not include inert species $(Q)$, or those which concentration were negligible for all reaction systems $(N)$. Considering the $N$ molecule produced by direct TMSD (Supplementary equation 4) as a distinct species greatly increased the complexity of the ODE model that results from this reaction network. Since $k_{r}$ and $k_{b}$ are always large, we assume for simplicity that direct TMSD instead produces the complex $N / I T$, which swiftly binds to the reporter (Supplementary equations 7 and 10). 
The subsequent detachment of $I T$ from the triggered reporter complex $R N$ is then accounted for by the backwards reaction in Supplementary equation 8.

The introduced approximation could fail when both the toehold and handhold are long. In this limit, toehold-based displacements will produce $N$ quicker, and this $N$ can sequester 1 -strands with its handhold, either before or after triggering the reporter. In our approximation, the $N$ species will instead remain bound to the reacted invader-target complex and cannot perform sequestration of the unreacted invader. However, regions of long proximal toeholds are not the primary focus of this work, and any discrepancies introduced are likely small on the scale of over four orders of magnitude considered here.

No base-pair-level intermediates were considered explicitly for any of the branch migration reactions. The resultant ODE model was composed of the mass-action law based differential equations:

$\frac{d[T N / I]}{d t}=k_{b}[T N][I]-k_{d}[T N / I]-k_{m}[T N / I],(12)$
$\frac{d[T N / I T]}{d t}=k_{b}[T N][I T]-k_{d}[T N / I T]-k_{t}[T N / I T][I]$,

$\frac{d[N / I T]}{d t}=k_{m}[T N / I]+k_{t}[T N][I]+k_{t}[T N / I T][I]-k_{r}[N / I T][R Q]+k_{t}[R N / I][T N]+k_{t}[R N / I][T N / I T]$,

$\frac{d[I T]}{d t}=k_{t}[T N / I T][I]+k_{d}[T N / I T]+k_{d}[R N / I T]-k_{b}[T N][I T]-k_{b}[R N][I T]$,

$\frac{d[R N]}{d t}=k_{d}[R N / I]+k_{d}[R N / I T]-k_{b}[R N][I]-k_{b}[R N][I T]+k_{t}[R N / I][T N]$,

$\frac{d[R N / I]}{d t}=k_{b}[R N][I]+k_{b}[R N][I T]-k_{d}[R N / I]-k_{d}[R N / I T]-k_{t}[R N / I][T N]-k_{t}[R N / I][T N / I T]$,

$\frac{d[R N / I T]}{d t}=k_{r}[N / I T][R Q]+k_{b}[R N][I T]-k_{d}[R N / I T]+k_{t}[R N / I][T N / I T]$.

With conservation laws for the initial species:

$[I]=[/]_{0}-[T N / I]-[T N / I T]-[N / I T]-[I T]-[R N / I]-[R N / I T]$,

$[T N]=[T N]_{0^{-}}[T N / I]-2[T N / I T]-[N / I T]-[I T]-[R N / I T],(20)$

$[R Q]=[R Q]_{0^{-}}[R N]-[R N / I]-[R N / I T]$

And initial conditions: $[T N / I]_{0},[T N / I T]_{0},[N / I T]_{0},[I T]_{0},[R N / I]_{0},[R N / I T]_{0}=0$ and $[R N]=[N]_{\text {excess, }}$ being $[N]_{\text {excess }}$ the excess of $N$ resultant from the inexact stoichiometry when annealing $T N$ with a $10 \%$ excess of $N$. $[N]_{\text {excess, }}[]_{0},[N]_{0}=[T N]_{0}+[N]_{0}$ and $[R Q]_{0}$ were inferred from the fluorescence signal produced during characterisation experiments as described in Supplementary Note I. 


\section{Supplementary Note XII:General fitting procedure}

All fluorescence data were first corrected as described in Supplementary Note VI and the baseline produced by $[N]_{\text {excess }}$ subtracted. All fitting procedures included, in addition to their rate constants, the reaction trigger concentration and $t_{0}$ for each trajectory.

The initial guesses for the reaction trigger concentrations were inferred from the fluorescence value of measurement 4 . The last value of the recorded kinetics in measurement 3 was compared with measurement 4 to determine the percentage of completion of the reaction during the kinetics recording. For kinetics that did not reach at least $90 \%$ completion, the concentration of reaction trigger was only allowed to fluctuate $\pm 10 \%$ from its initial guess.

The initial guess for $t_{0}\left(t_{0}\right.$ init $)$ was obtained from the cutting point of a regression line with the $x$-axis, except during reporter $R Q$ fitting (Supplementary Note XIII). The regression line was calculated by fitting the values of the kinetics that were under $50 \%$ completion, where the trajectories were almost linear.

Fittings were performed with the MATLAB ${ }^{\circledR}$ function fminsearch, based on the simplex search method by Lagarias et al. ${ }^{6}$ The common logarithm of the error function was minimised for all the fluorescence traces of a given system:

$$
\left.\log _{10} \text { (mean weighted error }\right)=\log _{10} \frac{\sum_{i} \sum_{t} \frac{\left(f_{\text {exp }}(i, t)-f_{\text {model }}(i, t)\right)^{2}}{f_{\text {model }}(i, t)}}{n}
$$

where $f_{\text {exp }}(i, t)$ is the inferred concentration at time $t$ of the trace $i$, and $f_{\text {model }}(i, t)$ is the sum of concentrations of all fluorescent species predicted by the model at time $t$ at the reagent conditions of the trace $i$. The error at each point was weighted by $f_{\text {model }}(i, t)$ to account for the Poisson distribution of the photomultiplier detection error as done by Zhang et al. ${ }^{3}$. When more than one trace was fitted simultaneously, the mean total weighted error was calculated by dividing the sum of the error by the number of fitted traces $(n)$.

All MATLAB fitting scripts are available for download at www.zenodo.org/record/4389212. 


\section{Supplementary Note XIII: $\quad$ Reporter $R Q$ fitting}

The experimental value of $k_{r}$ was obtained for every species of $N$ used during the characterisation experiments. Equation $\mathbf{8}$ in the main text was fitted to each trajectory individually. Since all reactions were completed in a minute, the effect of the initial leak on $t_{0}$ was not significant. Thus, the time of $N / I T$ injection was considered $t_{0 \_ \text {init. }}$

The model was fitted in a three-step iteration. During the $1^{\text {st }}$ round of fittings, the initial guess $k_{r_{-} \text {init }}$ was inferred from the time at which the reaction reached $90 \%$ completion:

$$
k_{r_{-} \text {init }}=\frac{\ln \left(10-9 \frac{[N]_{0}}{[R Q]_{0}}\right)}{t_{90 \%}\left([R Q]_{0}-[N]_{0}\right)},
$$

with $t_{90 \%}$ being the time at which $90 \%$ of $[/]_{0}$ reacted, and $[N]_{0}$ the total amount of strand $N$ injected in the form of $N / I T$. During the $2^{\text {nd }}$ round of fittings, $k_{r_{-} \text {init }}$ was the mean of all the $k_{r}$ obtained during the first round at the same temperature $\left(k_{r_{-} \text {init }}\right.$ at $25^{\circ} \mathrm{C}: 5.10^{6} \mathrm{~s}^{-1} \mathrm{M}^{-1} ; k_{r_{-} \text {init }}$ at $\left.37^{\circ} \mathrm{C}: 4.8 .10^{6} \mathrm{~s}^{-1} \mathrm{M}^{-1}\right)$. For the $3^{\text {rd }}$ round, all systems were fitted using as initial guesses their results of the second round for $k_{r}, t_{0}$ and $[N]_{0}$. All fitted $k_{r}$ at $25^{\circ} \mathrm{C}$ and $37^{\circ} \mathrm{C}$ are collected in Supplementary Tables 9 and 10, respectively

\section{Supplementary Table $9 \mid$ Fitted $k_{r}$ at $25^{\circ} \mathrm{C}$}

Data obtained using procedures described in Supplementary Notes I and XIII. The reactant strands used were all $N$ strands "Incumbent $\mathrm{x}$ hh/x sp" (Supplementary Table 20) with "Target (7 th)" (Supplementary Table 21) and "Invader ( $\left.I_{h c}\right)$ " (Supplementary Table 22). Reporter strands were "Reporter $1(R)^{\prime}$ " and "Reporter 1 quencher (Q)" (Supplementary Table 23). Mean of all fitted $k_{r}=$ $5.068 .10^{6} \mathrm{~s}^{-1} \mathrm{M}^{-1} ;$ Standard deviation $=0.505 .10^{6} \mathrm{~s}^{-1} \mathrm{M}^{-1} ;$ Standard mean error $(\mathrm{SEM})=0.052 .10^{6} \mathrm{~s}^{-1} \mathrm{M}^{-1}$.

\begin{tabular}{|c|c|c|c|c|c|}
\hline \multirow{2}{*}{$\begin{array}{c}\begin{array}{c}\text { Condition } \\
\text { (handhold/spacer) }\end{array} \\
0 / 0\end{array}$} & \multicolumn{4}{|c|}{$\begin{array}{c}\text { Individual experiments } \\
\qquad k_{r} \cdot 10^{6}\left(s^{-1} M^{-1}\right)\end{array}$} & \multirow{2}{*}{$\begin{array}{c}\text { Mean } k_{r .10^{6} \pm S E M} \\
\left(s^{-1} M^{-1}\right)\end{array}$} \\
\hline & 5.46 & 5.42 & 5.55 & 5.60 & \\
\hline $0 / 1$ & 5.41 & 6.11 & 5.54 & 6.02 & $5.77 \pm 0.17$ \\
\hline $0 / 2$ & 5.18 & 5.18 & 5.24 & 5.09 & $5.17 \pm 0.03$ \\
\hline $0 / 3$ & 5.45 & 4.99 & 5.08 & 5.04 & $5.14 \pm 0.1$ \\
\hline $0 / 5$ & 5.42 & 5.63 & 5.63 & 5.79 & $5.617 \pm 0.076$ \\
\hline $0 / 8$ & 5.51 & 5.29 & 5.43 & 5.21 & $5.359 \pm 0.069$ \\
\hline $4 / 2$ & 5.47 & 5.25 & 5.44 & 4.08 & $5.06 \pm 0.33$ \\
\hline $5 / 0$ & 5.32 & 5.06 & 5.63 & 6.11 & $5.55 \pm 0.22$ \\
\hline $5 / 1$ & 3.95 & 4.21 & 4.27 & 4.58 & $4.25 \pm 0.13$ \\
\hline $5 / 2$ & 4.66 & 4.35 & 4.33 & 5.68 & $4.76 \pm 0.32$ \\
\hline $5 / 3$ & 5.42 & 5.69 & 4.49 & 5.38 & $5.24 \pm 0.26$ \\
\hline $5 / 5$ & 5.63 & 5.61 & 5.98 & 5.69 & $5.728 \pm 0.086$ \\
\hline $5 / 8$ & 5.08 & 4.63 & 5.03 & 4.65 & $4.85 \pm 0.12$ \\
\hline $6 / 2$ & 4.46 & 4.91 & 4.53 & 4.52 & $4.61 \pm 0.1$ \\
\hline $7 / 2$ & 5.00 & 4.85 & 4.94 & 5.10 & $4.97 \pm 0.51$ \\
\hline $8 / 2$ & 4.82 & 4.55 & 4.72 & 5.05 & $4.78 \pm 0.1$ \\
\hline $9 / 2$ & 4.60 & 4.54 & 4.71 & 4.83 & $4 . .67 \pm 0.064$ \\
\hline $10 / 2$ & 4.80 & 4.83 & 6.01 & 4.80 & $5.11 \pm 0.30$ \\
\hline $20 / 0$ & 3.97 & 4.13 & 3.77 & 4.14 & $4.004 \pm 0.087$ \\
\hline $20 / 1$ & 5.24 & 5.06 & 5.45 & 5.31 & $5.265 \pm 0.081$ \\
\hline $20 / 2$ & 4.87 & 4.96 & 5.11 & 4.75 & $4.922 \pm 0.078$ \\
\hline $20 / 3$ & 4.91 & 5.01 & 5.00 & 4.80 & $4.931 \pm 0.049$ \\
\hline $20 / 5$ & 5.13 & 5.06 & 5.47 & 5.58 & $5.31 \pm 0.13$ \\
\hline $20 / 8$ & 5.12 & 5.12 & 5.12 & 4.9 & $5.08 \pm 0.041$ \\
\hline
\end{tabular}




\section{Supplementary Table 10| Fitted $\boldsymbol{k}_{r}$ at $37^{\circ} \mathrm{C}$}

Data obtained using procedures described in Supplementary Notes I and XIII. The reactant strands used were all $N$ strands "Incumbent $\mathrm{x} \mathrm{hh/x} \mathrm{sp"} \mathrm{except} \mathrm{the} \mathrm{ones} \mathrm{with} \mathrm{spacer} \mathrm{1,3,5} \mathrm{nt} \mathrm{(Supplementary}$ Table 20) with "Target (7 th)" (Supplementary Table 21) and "Invader ( $\left.I_{h c}\right)$ " (Supplementary Table 22). Reporter strands were "Reporter $1(R)$ " and "Reporter 1 quencher $(Q)$ " (Supplementary Table 23). Legend: Red highlight: $k_{r}$ values that were not obtained from direct experimental evidence. These values were estimated from the mean of all the obtained $k_{r}$ at $37^{\circ} \mathrm{C}$. Mean of all $k_{r}=4.52 .10^{6} \mathrm{~s}^{-1} \mathrm{M}^{-1}$; Standard deviation $=0.53 .10^{6} \mathrm{~s}^{-1} \mathrm{M}^{-1} ;$ Standard mean error $(\mathrm{SEM})=0.11 .10^{6} \mathrm{~s}^{-1} \mathrm{M}^{-1}$

\begin{tabular}{|c|c|c|c|c|c|}
\hline $\begin{array}{c}\text { Condition } \\
\text { (handhold/spacer) }\end{array}$ & \multicolumn{4}{|c|}{$\begin{array}{l}\text { Individual experiments } \\
\qquad k_{r} 10^{6}\left(s^{-1} M^{-1}\right)\end{array}$} & $\begin{array}{c}\text { Mean } k_{r} .10^{6} \pm S E M \\
\left(s^{-1} M^{-1}\right)\end{array}$ \\
\hline $0 / 0$ & 4.38 & 4.61 & 4.92 & 5.15 & $4.76 \pm 0.17$ \\
\hline $0 / 8$ & 4.66 & 4.87 & 4.93 & 5.27 & $4.94 \pm 0.13$ \\
\hline $4 / 2$ & - & - & - & - & $4.52 \pm 0.11$ \\
\hline $5 / 0$ & 4.65 & 4.57 & 4.84 & 4.64 & $4.677 \pm 0.057$ \\
\hline $5 / 2$ & - & - & - & - & $4.52 \pm 0.11$ \\
\hline $5 / 8$ & 4.07 & 4.26 & 4.54 & 4.88 & $4.44 \pm 0.18$ \\
\hline $6 / 2$ & - & - & - & - & $4.52 \pm 0.11$ \\
\hline $7 / 2$ & - & - & - & - & $4.52 \pm 0.11$ \\
\hline $8 / 2$ & - & - & - & - & $4.52 \pm 0.1$ \\
\hline $9 / 2$ & - & - & - & - & $4.52 \pm 0.11$ \\
\hline $10 / 2$ & - & - & - & - & $4.52 \pm 0.11$ \\
\hline $20 / 0$ & 3.29 & 3.52 & 3.76 & 3.54 & $3.525 \pm 0.096$ \\
\hline $20 / 2$ & - & - & - & - & $4.52 \pm 0.11$ \\
\hline $20 / 8$ & 4.63 & 4.84 & 4.76 & 4.95 & $4.794 \pm 0.069$ \\
\hline
\end{tabular}




\section{Supplementary Note XIV: $\quad$ TMSD fitting}

The value of $k_{t}$ was fitted using the individual trajectories of each system with a simplified ODE model consisting of Supplementary equations 14 and 18, assuming $[T N / I T], k_{b}, k_{d}$, and $k_{m}=0$.

$$
\begin{gathered}
\frac{d[N]}{d t}=k_{t}[T N][I]-k_{r}[N][R Q], \\
\frac{d[R N]}{d t}=k_{r}[N][R Q] .(25)
\end{gathered}
$$

The model was fitted iteratively in three steps. During the $1^{\text {st }}$ round of fittings, $k_{t}$ init was obtained using a modification of Supplementary equation 23 , exchanging $[N]_{0}$ for $[I]_{0}$, and $[R Q]_{0}$ for $[T N]_{0}$. For kinetics that did not reach $90 \%$ of completion, $k_{t_{-} \text {init }}$ was obtained from the generalized form of Supplementary equation 23:

$$
k_{t_{-} \text {init }}=\frac{\ln \left(\frac{[I]_{0}}{[I]_{\text {end }}}+\frac{[I]_{0}}{[T N]_{0}}\left(1-\frac{[I]_{0}}{[I]_{\text {end }}}\right)\right)}{t_{\text {end }}[T N]_{0}-[I]_{0}},
$$

with $[l]_{\text {end }}$ being the maximum [l] value recorded during the kinetics and $t_{\text {end }}$ the final time of the measurement (corrected with $t_{o_{-} \text {init }}$ ). During the $2^{\text {nd }}$ round of fittings, $k_{\_}$init was the median of all the $k_{t}$ obtained for that system during the $1^{\text {st }}$ round. For the $3^{\text {rd }}$ round, all systems were fitted using as initial guesses its results of the second round for $k_{t}, t_{0}$ and $[I]_{0}$

Reactions triggered with $I_{h c}$, but in the absence of handholds in $N$ also followed this fitting procedure to confirm the value of $k_{t}$ obtained with $I_{w c}$. All fitted $k_{t}$ are collected in Supplementary Tables $\mathbf{1 5}$ to 17. 


\section{Supplementary Note XV: $k_{b}$ estimation}

For reaction systems located in the $2^{\text {nd }}$ order regime with irreversible handhold-binding (handhold $=20$ $\mathrm{nt}$; toehold $>1 \mathrm{nt}), k_{d}$ is considered negligible and $k_{\mathrm{m}}$ very large. Thus, the reaction rate is solely determined by $k_{b}$ (equation 4). To obtain an estimation of $k_{b}$, the complete ODE model, described in Supplementary Note XI, was fitted to individual fluorescence traces of systems confidently located in the $2^{\text {nd }}$ order regime ( $20 \mathrm{nt}$ handhold; $>1$ nt toehold).

During the fitting, $k_{m}$ was fixed to an arbitrarily large value $\left(10^{8} \mathrm{~s}^{-1}\right)$ and $k_{d}$ to $1.10^{-22} \mathrm{~s}^{-1}$. The data was fitted in a two-step iteration. During the $1^{\text {st }}$ round of fittings, $k_{b}$ init was arbitrarily set to $10^{7} \mathrm{~s}^{-1} \mathrm{M}^{-1}$. For the $2^{\text {nd }}$ round, all systems were fitted using as initial guesses its results of the $1^{\text {st }}$ round for $k_{b}, t_{0}$ and $[/]_{0}$.

For the subsequent fittings, the value of $k_{b}$ at each temperature was constrained to a range of twice the standard deviation of the fitted $k_{b}$, centred on its mean value. All $k_{b}$ and their ranges at $25^{\circ} \mathrm{C}$ and $37^{\circ} \mathrm{C}$ are collected in Supplementary Tables 11 and 12, respectively.

\section{Supplementary Table 11| $k_{b}$ fitting at $25^{\circ} \mathrm{C}$}

Data obtained using procedures described in Supplementary Notes II and XV. The reactant strands used were all $N$ strands "Incumbent $20 \mathrm{hh} / \mathrm{x} \mathrm{sp"} \mathrm{(Supplementary} \mathrm{Table} \mathrm{20)} \mathrm{with} T$ strands "Target (x th)" from 2 to 7 nt (Supplementary Table 21) and "Invader ( $\left.I_{h c}\right)$ " (Supplementary Table 22). Reporter strands were "Reporter $1(R)^{\prime \prime}$ " and "Reporter 1 quencher $(Q)$ " (Supplementary Table 23). Mean of all $k_{b}=5 \cdot 14 \cdot 10^{6} \mathrm{~s}^{-1} \mathrm{M}^{-1} ;$ Standard deviation $=1.27 .10^{6} \mathrm{~s}^{-1} \mathrm{M}^{-1} ;$ Standard mean error $(\mathrm{SEM})=0.17 .10^{6} \mathrm{~s}^{-1} \mathrm{M}^{-1}$.

\begin{tabular}{|c|c|c|c|c|c|}
\hline $\begin{array}{c}\text { Condition } \\
\text { (handhold/spacer/toehold) }\end{array}$ & \multicolumn{4}{|c|}{$\begin{array}{l}\text { Individual experiments } \\
\qquad k_{b} 10^{6}\left(\mathrm{~s}^{-1} \mathrm{M}^{-1}\right)\end{array}$} & $\begin{array}{c}\text { Mean } k_{b} 10^{6} \pm S E M \\
\left(s^{-1} M^{-1}\right)\end{array}$ \\
\hline $20 / 0 / 7$ & 6.41 & 6.31 & 5.87 & 7.87 & $6.62 \pm 0.44$ \\
\hline $20 / 0 / 2$ & 3.47 & 3.49 & 3.21 & 3.51 & $3.421 \pm 0.071$ \\
\hline $20 / 1 / 7$ & 7.68 & 6.87 & 6.92 & 6.83 & $7.07 \pm 0.2$ \\
\hline $20 / 1 / 2$ & 5.44 & 5.22 & 5.77 & 5.24 & $5.42 \pm 0.13$ \\
\hline $20 / 2 / 7$ & 5.74 & 5.74 & 5.44 & 5.95 & $5.72 \pm 0.1$ \\
\hline $20 / 2 / 5$ & 7.60 & 7.66 & 7.88 & 6.92 & $7.52 \pm 0.21$ \\
\hline $20 / 2 / 3$ & 4.91 & 4.86 & 4.87 & 4.47 & $4.78 \pm 0.1$ \\
\hline 20/2/2 & 5.55 & 5.28 & 5.52 & 5.29 & $5.411 \pm 0.071$ \\
\hline 20/3/7 & 6.22 & 4.89 & 5.00 & 4.52 & $5.16 \pm 0.37$ \\
\hline $20 / 3 / 2$ & 4.90 & 4.69 & 4.59 & 4.46 & $4.659 \pm 0.092$ \\
\hline $20 / 5 / 7$ & 3.87 & 3.55 & 3.61 & 3.40 & $3.609 \pm 0.098$ \\
\hline $20 / 5 / 2$ & 5.17 & 4.23 & 4.39 & 3.98 & $4.44 \pm 0.26$ \\
\hline $20 / 8 / 7$ & 4.12 & 3.99 & 4.19 & 4.12 & $4.105 \pm 0.044$ \\
\hline $20 / 8 / 2$ & 4.47 & 3.82 & 3.84 & 4.08 & $4.05 \pm 0.15$ \\
\hline
\end{tabular}

\section{Supplementary Table $12 \mid k_{b}$ fitting at $37^{\circ} \mathrm{C}$}

Data obtained using procedures described in Supplementary Notes II and XV. The reactant strands used were all $N$ strands "Incumbent $20 \mathrm{hh} / \mathrm{x}$ sp" (Supplementary Table 20) with $T$ strands "Target (x th)" from 2 to 7 nt (Supplementary Table 21) and "Invader ( $\left.I_{h c}\right)$ " (Supplementary Table 22). Reporter strands were "Reporter $1(R)$ " and "Reporter 1 quencher $(Q)$ " (Supplementary Table 23). Legend: Red: Rates with a standard error bigger than the $10 \%$ of the mean. Corresponds with long experimental conditions (toehold $=0 \mathrm{nt}$ ) or systems at the boundary between different kinetic regimes. Mean of all $k_{b}=1.10^{7} \mathrm{~s}^{-1} \mathrm{M}^{-1} ;$ Standard deviation $=0.45 \cdot 10^{7} \mathrm{~s}^{-1} \mathrm{M}^{-1} ;$ Standard mean error $(\mathrm{SEM})=0.11 .10^{7} \mathrm{~s}^{-1} \mathrm{M}^{-1}$.

\begin{tabular}{|c|c|c|c|c|c|}
\hline $\begin{array}{c}\text { Condition } \\
\text { (handhold/spacer) }\end{array}$ & \multicolumn{4}{|c|}{$\begin{array}{l}\text { Individual experiments } \\
\qquad k_{b} 10^{6}\left(s^{-1} M^{-1}\right)\end{array}$} & $\begin{array}{c}\text { Mean } k_{b} 10^{6} \pm S E M \\
\left(s^{-1} M^{-1}\right)\end{array}$ \\
\hline $20 / 0 / 7$ & 13.73 & 15.82 & 17.81 & 17.84 & $16.3 \pm 0.98$ \\
\hline $20 / 0 / 2$ & 10.47 & 10.66 & 10.18 & 11.25 & $10.64 \pm 0.23$ \\
\hline $20 / 8 / 7$ & 6.09 & 6.68 & 2.90 & 3.07 & $4.69 \pm 0.99$ \\
\hline $20 / 8 / 2$ & 7.94 & 8.98 & 9.23 & 8.59 & $8.68 \pm 0.28$ \\
\hline
\end{tabular}




\section{Supplementary Note XVI: HMSD fitting}

The parameters $k_{d}, k_{m}, k_{b},\left[l_{0}\right.$ and $t_{0}$ were fitted simultaneously for each set of HMSD trajectories. A single set of values for $k_{d}, k_{m}$ and $k_{b}$ was fitted for each system condition. Constraints for the value of $k_{b}$ and the value for the rest of the rate constants in the model were obtained experimentally (See Supplementary Notes XIII, XIV and XV). The model was fitted iteratively in three steps. During the $1^{\text {st }}$ round of fittings, $k_{d \_ \text {init }}$ was calculated with an estimation of handhold-binding free energy for each handhold and spacer combination ${ }^{1}$, using the equilibrium equation:

$$
k_{d_{-} i n i t}=\frac{k_{b}}{e^{-\left(\frac{\Delta G_{h b}}{R T}\right)}},
$$

where $R$ is the gas constant $\left(1.987 \mathrm{cal} \mathrm{mol}^{-1} \mathrm{~K}^{-1}\right), T$ is the experimental temperature, and $\Delta G_{h b}$ is the estimation of the handhold-binding Gibbs free energy in calories per mol ${ }^{1}$. All the estimated $\Delta G_{h b}$ and $k_{d \_ \text {init }}$ at $25^{\circ} \mathrm{C}$ and $37^{\circ} \mathrm{C}$ are collected in Supplementary Tables 13 and 14 , respectively. The value of $k_{d}$ was constrained during the fitting to a range $\pm \sqrt{10} k_{d \_ \text {init. }} k_{m_{-} \text {init }}$ was changed iteratively in the range of $\left[10^{-6}-10^{4}\right]$ one order of magnitude at a time, and the best fitting was stored. For the $2^{\text {nd }}$ round, each system was fitted without constraining $k_{d}$, using the best result of the $1^{\text {st }}$ fitting round as initial guesses for $k_{d}, k_{b}, k_{m}, t_{0}$ and []$_{0}$. The $3^{\text {rd }}$ fitting round used the results of the $2^{\text {nd }}$ fitting round as initial guesses. Results from this fitting procedure and their calculated reaction rates and half-lives at $25^{\circ} \mathrm{C}$ and $37^{\circ} \mathrm{C}$ are collected in Supplementary Tables 15, 16 and 17.

To estimate and error for the fitted constants, the model was fitted to each individual trajectory and the standard error of the mean of each rate is reported. The results of the previous fitting process were used as initial guesses for $k_{d}, k_{b}, k_{m}, t_{0}$ and $[/]_{0}$. For reactions with $2^{\text {nd }}$ order rate kinetics with reversible handhold binding, the standard error for the obtained $2^{\text {nd }}$ order rate $k$ (equation 4 ) is reported, due to compensatory errors between the ratio $\frac{k_{m}}{k_{m}+k_{d}}$ and $k_{b}$. For reactions with $2^{\text {nd }}$ order kinetics and irreversible handhold binding, the standard error for the obtained $k_{b}$ is reported. For reactions with $1^{\text {st }}$ order kinetics, the standard error for the obtained $k_{m}$ is reported. 


\section{Supplementary Table 13| Estimated $\Delta \mathbf{G}_{h b}$ and $k_{d}$ for each handhold at $25^{\circ} \mathrm{C}$}

Data obtained as described in Supplementary Notes II and XVI. The reactant strands used were all $N$ strands "Incumbent $\mathrm{x} \mathrm{hh/x} \mathrm{sp"} \mathrm{(Supplementary} \mathrm{Table} \mathrm{20)} \mathrm{with} \mathrm{all} T$ strands "Target ( $\mathrm{x}$ th)" (Supplementary Table 21) and "Invader $\left(I_{h c}\right)$ " (Supplementary Table 22). Reporter strands were "Reporter $1(R)$ " and "Reporter 1 quencher $(Q)$ " (Supplementary Table 23).

\begin{tabular}{|c|c|c|c|c|c|c|c|c|c|}
\hline $\begin{array}{c}\text { Condition } \\
\text { (handhold/spacer) }\end{array}$ & $\begin{array}{c}\Delta \mathbf{G}_{h b} \\
\left(K c a l M^{-1}\right)\end{array}$ & $\begin{array}{c}k_{d \_} \text {init } \\
\left(s^{-1}\right)\end{array}$ & \multicolumn{6}{|c|}{$\begin{array}{l}\text { Mean fitted } k_{d} \text { for each condition } \\
\qquad\left(s^{-1}\right)\end{array}$} & $\begin{array}{c}\text { Mean kd } \\
\pm \operatorname{SEM}\left(s^{-1}\right) \\
\end{array}$ \\
\hline $4 / 2$ & -7.24 & $2.48 \mathrm{E}+01$ & $7.82 \mathrm{E}+00$ & $1.29 \mathrm{E}+01$ & $1.13 \mathrm{E}+01$ & $3.07 E+01$ & $4.03 E+01$ & $8.43 E+00$ & $1.86 \pm 0.55 \mathrm{E}+1$ \\
\hline $5 / 0$ & -7.26 & $2.40 \mathrm{E}+01$ & $1.34 \mathrm{E}+01$ & - & - & $2.11 \mathrm{E}+01$ & - & $7.67 \mathrm{E}+00$ & $1.41 \pm 0.39 \mathrm{E}+1$ \\
\hline $5 / 1$ & -7.96 & $7.35 \mathrm{E}+00$ & $2.11 \mathrm{E}+00$ & - & - & $7.80 \mathrm{E}+02$ & - & $2.57 \mathrm{E}+00$ & $2.6 \pm 2.6 \mathrm{E}+2$ \\
\hline $5 / 2$ & -8.03 & $6.53 \mathrm{E}+00$ & $8.27 E+00$ & $1.70 \mathrm{E}+01$ & $3.19 E+00$ & $1.96 \mathrm{E}+01$ & $9.81 \mathrm{E}+00$ & $2.46 \mathrm{E}+00$ & $1.01 \pm 0.29 \mathrm{E}+1$ \\
\hline $5 / 3$ & -7.96 & $7.35 \mathrm{E}+00$ & $2.63 E+00$ & - & - & $9.16 \mathrm{E}+00$ & - & $9.56 \mathrm{E}+00$ & $7.1 \pm 2.2 \mathrm{E}+0$ \\
\hline $5 / 5$ & -7.74 & $1.07 \mathrm{E}+01$ & $6.00 E+00$ & - & - & $7.62 \mathrm{E}+00$ & - & 1.37E-01 & $4.6 \pm 2.3 E+0$ \\
\hline $5 / 8$ & -7.96 & $7.35 \mathrm{E}+00$ & $2.59 \mathrm{E}+00$ & - & - & $4.70 \mathrm{E}+00$ & - & $2.79 \mathrm{E}+00$ & $3.36 \pm 0.67 \mathrm{E}+0$ \\
\hline $6 / 2$ & -10.22 & $1.62 \mathrm{E}-01$ & $5.14 \mathrm{E}-01$ & $1.56 \mathrm{E}-01$ & $2.87 \mathrm{E}-02$ & 2.07E-01 & $8.92 \mathrm{E}-01$ & 7.95E-02 & $3.1 \pm 1.4 \mathrm{E}-1$ \\
\hline $7 / 2$ & -11.18 & $3.21 \mathrm{E}-02$ & $1.63 \mathrm{E}-02$ & $5.90 \mathrm{E}-02$ & $9.77 \mathrm{E}-02$ & $4.72 \mathrm{E}-02$ & 2.07E-03 & $8.26 \mathrm{E}-02$ & $5.1 \pm 1.5 \mathrm{E}-2$ \\
\hline $8 / 2$ & -12.39 & $4.16 \mathrm{E}-03$ & $1.60 \mathrm{E}-02$ & $9.74 \mathrm{E}-03$ & $7.65 \mathrm{E}-02$ & $1.23 \mathrm{E}-01$ & $1.76 \mathrm{E}-02$ & 2.63E-04 & $4.1 \pm 2 \mathrm{E}-2$ \\
\hline $9 / 2$ & -15.04 & 4.75E-05 & $8.88 \mathrm{E}-14$ & 2.37E-11 & $9.01 \mathrm{E}-09$ & 6.09E-09 & $3.73 \mathrm{E}-09$ & $4.08 \mathrm{E}-06$ & $6.8 \pm 6.8 \mathrm{E}-7$ \\
\hline $10 / 2$ & -15.83 & $1.25 \mathrm{E}-05$ & $2.89 \mathrm{E}-12$ & $5.80 \mathrm{E}-03$ & $6.13 \mathrm{E}-03$ & 3.79E-03 & 4.90E-04 & $1.52 \mathrm{E}-03$ & $3 \pm 1.1 \mathrm{E}-3$ \\
\hline $20 / 0$ & -29.72 & $8.22 \mathrm{E}-16$ & $1.04 \mathrm{E}-20$ & - & - & $6.59 \mathrm{E}-16$ & - & $3.81 \mathrm{E}-16$ & $3.5 \pm 1.9 \mathrm{E}-16$ \\
\hline $20 / 1$ & -30.42 & $2.52 \mathrm{E}-16$ & $3.01 \mathrm{E}-16$ & - & - & $3.72 \mathrm{E}-16$ & - & 1.17E-16 & $2.63 \pm 0.76 \mathrm{E}-16$ \\
\hline $20 / 2$ & -30.5 & $2.20 \mathrm{E}-16$ & 2.39E-16 & $6.68 \mathrm{E}-16$ & $1.46 \mathrm{E}-16$ & $5.41 \mathrm{E}-16$ & 8.03E-17 & $8.14 \mathrm{E}-17$ & $2.9 \pm 1 \mathrm{E}-16$ \\
\hline $20 / 3$ & -30.42 & $2.52 \mathrm{E}-16$ & $9.14 \mathrm{E}-17$ & - & - & 4.10E-16 & - & $1.27 \mathrm{E}-16$ & $2.1 \pm 1 E-16$ \\
\hline $20 / 5$ & -30.21 & $3.59 \mathrm{E}-16$ & $1.69 \mathrm{E}-16$ & - & - & $2.58 \mathrm{E}-16$ & - & $1.67 \mathrm{E}-16$ & $1.98 \pm 0.3 \mathrm{E}-16$ \\
\hline \multirow[t]{2}{*}{$20 / 8$} & -30.42 & $2.52 \mathrm{E}-16$ & $2.65 \mathrm{E}-16$ & - & - & 5.73E-16 & - & 2.17E-16 & $3.5 \pm 1.1 \mathrm{E}-16$ \\
\hline & \multicolumn{2}{|c|}{ Toehold lengths } & $7 n t$ & $5 n t$ & $3 n t$ & $2 n t$ & $1 \mathrm{nt}$ & $0 \mathrm{nt}$ & \\
\hline
\end{tabular}

\section{Supplementary Table 14 Estimated $\Delta \mathbf{G}_{h b}$ and $\boldsymbol{k}_{d}$ for each handhold at $37^{\circ} \mathrm{C}$}

Data obtained as described in Supplementary Notes II and XVI. The reactant strands used were all $N$ strands "Incumbent $\mathrm{x} \mathrm{hh} / \mathrm{x} \mathrm{sp}$ " except the ones with spacer 1,3 and $5 \mathrm{nt}$ (Supplementary Table 20) with "Target ( $x$ th)" with 0, 2 and 8 nt (Supplementary Table 21) and "Invader ( $\left.I_{h c}\right)$ " (Supplementary Table 22). Reporter strands were "Reporter $1(R)$ " and "Reporter 1 quencher $(Q)$ " (Supplementary Table 23).

\begin{tabular}{|c|c|c|c|c|c|c|}
\hline $\begin{array}{c}\text { Condition } \\
\text { (handhold/spacer) }\end{array}$ & $\begin{array}{c}\Delta \mathbf{G h b} \\
\left(K_{\text {Kcal M}}^{-1}\right)\end{array}$ & $\begin{array}{c}\text { kd_init } \\
\left(s^{-1}\right)\end{array}$ & \multicolumn{3}{|c|}{$\begin{array}{l}\text { Fitted } \boldsymbol{k}_{\boldsymbol{d}} \\
\qquad\left(\mathrm{s}^{-1}\right)\end{array}$} & $\begin{array}{c}\text { Mean } k_{d} \\
\pm \operatorname{SEM}\left(s^{-1}\right)\end{array}$ \\
\hline $4 / 2$ & -6.02 & $5.72 \mathrm{E}+02$ & - & - & $1.95 E+02$ & $1.95 \mathrm{E}+02$ \\
\hline $5 / 0$ & -6.26 & $3.88 \mathrm{E}+02$ & $3.37 E+02$ & $1.82 \mathrm{E}+02$ & $1.28 \mathrm{E}+02$ & $2.16 \pm 0.51 \mathrm{E}+02$ \\
\hline $5 / 2$ & -6.87 & $1.44 E+02$ & - & - & $5.01 E+01$ & $5.01 \mathrm{E}+01$ \\
\hline $5 / 8$ & -6.84 & $1.51 E+02$ & $1.19 E+02$ & $3.59 \mathrm{E}+01$ & $4.79 E+01$ & $6.8 \pm 2.1 \mathrm{E}+01$ \\
\hline $6 / 2$ & -8.53 & $9.75 E+00$ & - & - & $9.13 E+00$ & $9.13 E+00$ \\
\hline $7 / 2$ & -9.45 & $2.19 E+00$ & - & - & $3.8 \mathrm{E}+00$ & $3.8 \mathrm{E}+00$ \\
\hline $8 / 2$ & -10.22 & $6.28 \mathrm{E}-01$ & - & - & $1.05 E+00$ & $1.05 \mathrm{E}+00$ \\
\hline $9 / 2$ & -12.49 & $1.59 \mathrm{E}-02$ & - & - & $1.37 \mathrm{E}-02$ & $1.37 \mathrm{E}-02$ \\
\hline $10 / 2$ & -13.34 & $3.98 \mathrm{E}-03$ & - & - & $1.53 \mathrm{E}-03$ & $1.53 \mathrm{E}-03$ \\
\hline $20 / 0$ & -24.65 & $4.26 \mathrm{E}-11$ & 4.36E-10 & $1.04 \mathrm{E}-10$ & $1.2 \mathrm{E}-11$ & $1.8 \pm 1.1 \mathrm{E}-10$ \\
\hline $20 / 2$ & -25.26 & $1.58 \mathrm{E}-11$ & - & - & $1.6 \mathrm{E}-11$ & $1.6 \mathrm{E}-11$ \\
\hline \multirow[t]{2}{*}{$20 / 8$} & -25.23 & $1.66 \mathrm{E}-11$ & $6.98 \mathrm{E}-11$ & $1.52 \mathrm{E}-06$ & $8.7 \mathrm{E}-12$ & $5.1 \pm 4.14 \mathrm{E}-07$ \\
\hline & \multicolumn{2}{|c|}{ Toehold lengths } & $7 n t$ & $2 n t$ & O nt & \\
\hline
\end{tabular}




\section{Supplementary Table 15| Handhold Characterisation at $25^{\circ} \mathrm{C}$}

Data used for Figure $\mathbf{3}$ in the main text. All the relevant characterisation constants are accompanied by the standard error of the mean of individual fittings (Supplementary Note XVI). Data obtained as described in Supplementary Notes II, XIV (to obtain $\boldsymbol{k}_{t}$ ) and XVI. The reactant strands used were all $N$ strands "Incumbent $\mathrm{x}$ hh/2 sp" (Supplementary Table 20) with all "Target ( $x$ th)" (Supplementary Table 21) and I strands "Invader $\left(I_{h c}\right)$ ", and to obtain the value of $k_{t}$ "Invader random-5' $\left(I_{w c}\right)$ " (Supplementary Table 22). Reporter strands were "Reporter $1(R)$ " and "Reporter 1 quencher $(Q)$ " (Supplementary Table 23). Legend: Bold: Relevant reaction rate for the system. Yellow highlight: $1^{\text {st }}$ order reaction rates. Green: $k_{b}$ with a negligible standard error of the mean. This artefact is produced by the boundaries imposed to $k_{b}$ during its fitting. Red: Rates with a standard error bigger than the $10 \%$ of the mean. Corresponds to long experimental conditions (toehold=0 nt) or systems at the boundary between different kinetic regimes. Red highlight: $k_{t}$ values that were not obtained from direct experimental evidence but the mean of all the systems with the same toehold length. Experiments omitted to reduce the number of extremely slow experiments. The standard error of the mean for each parameter was calculated from the values obtained in individual fittings.

\begin{tabular}{|c|c|c|c|c|c|c|c|}
\hline $\begin{array}{c}\text { System } \\
\text { hh/sp/toe }\end{array}$ & $\begin{array}{l}\boldsymbol{k}_{\boldsymbol{m}} \\
\left(\mathrm{s}^{-1}\right)\end{array}$ & $\begin{array}{c}\boldsymbol{k}_{\boldsymbol{d}} \\
\left(s^{-1}\right)\end{array}$ & $\begin{array}{c}\boldsymbol{k}_{\boldsymbol{b}} \\
\left(s^{-1} M^{-1}\right)\end{array}$ & $\begin{array}{c}\boldsymbol{k}_{t} \\
\left(s^{-1} M^{-1}\right)\end{array}$ & $\begin{array}{c}2^{\text {nd }} \text { order rate }(k) \\
\left(\text { eq.3) }\left(s^{-1} M^{-1}\right)\right.\end{array}$ & $\begin{array}{c}\boldsymbol{t}_{1 / 2}^{W c} \\
(\min )\end{array}$ & $\begin{array}{c}\boldsymbol{t}_{1 / 2} \\
\text { (min) }\end{array}$ \\
\hline $0 / 2 / 7$ & 0 & 0 & 0 & $2.942 \pm 0.085 \mathrm{E}+6$ & $2.942 \pm 0.085 \mathrm{E}+6$ & $5.45 \mathrm{E}-01$ & $5.45 \mathrm{E}-01$ \\
\hline $0 / 2 / 5$ & 0 & 0 & 0 & $2.101 \pm 0.037 E+5$ & $2.101 \pm 0.037 E+5$ & $6.56 \mathrm{E}+00$ & $6.56 \mathrm{E}+00$ \\
\hline $0 / 2 / 3$ & 0 & 0 & 0 & $1.267 \pm 0.025 E+4$ & $1.267 \pm 0.025 \mathrm{E}+4$ & $1.08 \mathrm{E}+02$ & $1.08 \mathrm{E}+02$ \\
\hline $0 / 2 / 2$ & 0 & 0 & 0 & $1.65 \pm 0.15 E+2$ & $1.65 \pm 0.15 E+2$ & $9.63 E+03$ & $9.63 \mathrm{E}+03$ \\
\hline $0 / 2 / 1$ & 0 & 0 & 0 & $8.59 \pm 0.26 E+1$ & $8.59 \pm 0.26 \mathrm{E}+1$ & $2.84 \mathrm{E}+04$ & $2.84 \mathrm{E}+04$ \\
\hline $4 / 2 / 7$ & $1.12 \mathrm{E}+0$ & $8.19 E+00$ & $6.41 E+06$ & $1.053 \pm 0.007 \mathrm{E}+6$ & $1.823 \pm 0.052 E+6$ & $1.77 \mathrm{E}+00$ & $7.80 \mathrm{E}-01$ \\
\hline $4 / 2 / 5$ & $9.52 \mathrm{E}-1$ & $1.26 \mathrm{E}+01$ & $5.24 \mathrm{E}+06$ & $1.136 \pm 0.009 \mathrm{E}+5$ & $4.82 \pm 0.11 \mathrm{E}+5$ & $1.23 \mathrm{E}+01$ & $2.93 \mathrm{E}+00$ \\
\hline $4 / 2 / 3$ & 3.37E-1 & $1.32 \mathrm{E}+01$ & $4.51 E+06$ & $1.599 \pm 0.011 E+4$ & $1.28 \pm 0.017 E+5$ & $8.77 \mathrm{E}+01$ & $1.10 \mathrm{E}+01$ \\
\hline $4 / 2 / 2$ & $1.34 \mathrm{E}-2$ & $2.42 \mathrm{E}+01$ & $5.72 E+06$ & $1.884 \pm 0.015 E+2$ & $3.357 \pm 0.066 E+3$ & $7.44 \mathrm{E}+03$ & $4.19 \mathrm{E}+02$ \\
\hline $4 / 2 / 1$ & $1.13 \mathrm{E}-2$ & $2.79 E+01$ & $4.62 \mathrm{E}+06$ & $1.097 \pm 0.045 \mathrm{E}+2$ & $1.984 \pm 0.076 \mathrm{E}+3$ & $1.28 \mathrm{E}+04$ & $7.08 \mathrm{E}+02$ \\
\hline $4 / 2 / 0$ & $1.78 \mathrm{E}-5$ & $7.55 \mathrm{E}+00$ & $4.76 \mathrm{E}+06$ & $8.3 \pm 1.3 \mathrm{E}+0$ & $1.95 \pm 0.41 \mathrm{E}+1$ & $1.70 \mathrm{E}+05$ & $7.31 \mathrm{E}+04$ \\
\hline $5 / 2 / 7$ & $1.79 \mathrm{E}+0$ & $8.57 E+00$ & $3.87 E+06$ & $6.123 \pm 0.093 E+5$ & $1.282 \pm 0.025 \mathrm{E}+6$ & $2.29 \mathrm{E}+00$ & $1.10 \mathrm{E}+00$ \\
\hline $5 / 2 / 5$ & $4.18 \mathrm{E}-2$ & $3.23 \mathrm{E}-01$ & $3.87 E+06$ & $8.69 \pm 0.16 \mathrm{E}+4$ & $4.958 \pm 0.068 E+5$ & $1.61 \mathrm{E}+01$ & $2.85 \mathrm{E}+00$ \\
\hline $5 / 2 / 3$ & 4.27E-1 & $8.46 \mathrm{E}+00$ & $5.24 E+06$ & $1.674 \pm 0.008 \mathrm{E}+4$ & $2.723 \pm 0.043 E+5$ & $1.82 \mathrm{E}+06$ & $5.29 \mathrm{E}+00$ \\
\hline $5 / 2 / 2$ & $9.66 \mathrm{E}-3$ & $7.28 \mathrm{E}+00$ & $3.87 E+06$ & $2.354 \pm 0.048 \mathrm{E}+2$ & $5.348 \pm 0.027 \mathrm{E}+3$ & $4.82 E+05$ & $2.63 E+02$ \\
\hline $5 / 2 / 1$ & $4.08 \mathrm{E}-3$ & $8.54 E+00$ & $6.41 \mathrm{E}+06$ & $1.116 \pm 0.006 E+2$ & $3.18 \pm 0.1 E+3$ & $1.26 \mathrm{E}+04$ & $4.45 \mathrm{E}+02$ \\
\hline $5 / 2 / 0$ & $1.49 \mathrm{E}-5$ & $2.11 \mathrm{E}+00$ & $5.33 E+06$ & $9.36 \pm 0.62 E+0$ & $4.607 \pm 0.064 E+1$ & $1.52 E+05$ & $3.13 E+04$ \\
\hline $6 / 2 / 7$ & $6.62 \mathrm{E}-1$ & $5.14 \mathrm{E}-01$ & $3.93 E+6$ & $1.072 \pm 0.021 \mathrm{E}+6$ & $3.285 \pm 0.06 E+6$ & $1.31 \mathrm{E}+00$ & $4.55 \mathrm{E}-01$ \\
\hline $6 / 2 / 5$ & $1.55 \mathrm{E}-1$ & $1.56 \mathrm{E}-01$ & $3.76 \mathrm{E}+6$ & $1.066 \pm 0.011 \mathrm{E}+5$ & $1.98 \pm 0.21 \mathrm{E}+6$ & $1.32 \mathrm{E}+01$ & $8.45 \mathrm{E}-01$ \\
\hline $6 / 2 / 3$ & $3.75 \mathrm{E}-2$ & 2.87E-02 & $3.76 \mathrm{E}+6$ & $2.135 \pm 0.015 E+4$ & $2.152 \pm 0.078 E+6$ & $6.57 E+01$ & $1.12 \mathrm{E}+00$ \\
\hline $6 / 2 / 2$ & $2.70 \mathrm{E}-3$ & 2.07E-01 & $3.77 \mathrm{E}+6$ & $2.641 \pm 0.029 \mathrm{E}+2$ & $4.87 \pm 0.12 E+4$ & $5.31 \mathrm{E}+03$ & $3.66 \mathrm{E}+01$ \\
\hline $6 / 2 / 1$ & $4.92 \mathrm{E}-3$ & 8.92E-01 & $4.04 \mathrm{E}+6$ & $1.335 \pm 0.01 \mathrm{E}+2$ & $2.227 \pm 0.037 E+4$ & $1.05 E+04$ & $6.74 \mathrm{E}+01$ \\
\hline $6 / 2 / 0$ & $7.18 \mathrm{E}-6$ & 7.95E-02 & $4.53 \mathrm{E}+6$ & $9.36 \pm 0.62 E+0$ & $4.19 \pm 0.18 \mathrm{E}+2$ & $1.52 \mathrm{E}+05$ & $6.03 \mathrm{E}+03$ \\
\hline $7 / 2 / 7$ & $8.16 \mathrm{E}-2$ & $1.63 \mathrm{E}-02$ & $3.76 \mathrm{E}+6$ & $9.99 \pm 0.17 \mathrm{E}+5$ & $4.13 \pm 0.13 E+6$ & $1.40 \mathrm{E}+00$ & 5.17E-01 \\
\hline $7 / 2 / 5$ & $1.92 \mathrm{E}-1$ & $5.90 \mathrm{E}-02$ & $3.76 \mathrm{E}+6$ & $1.146 \pm 0.009 \mathrm{E}+5$ & $2.991 \pm 0.031 E+6$ & $1.22 \mathrm{E}+01$ & $5.71 \mathrm{E}-01$ \\
\hline $7 / 2 / 3$ & $1.23 \mathrm{E}-1$ & $9.77 \mathrm{E}-02$ & $6.29 \mathrm{E}+6$ & $2.433 \pm 0.032 E+4$ & $3.53 \pm 0.11 E+6$ & $5.76 \mathrm{E}+01$ & $5.55 \mathrm{E}-01$ \\
\hline $7 / 2 / 2$ & $5.63 \mathrm{E}-3$ & 4.72E-02 & $3.76 \mathrm{E}+6$ & $2.987 \pm 0.045 E+2$ & $4 \pm 1.3 E+5$ & $4.69 \mathrm{E}+03$ & $6.87 E+00$ \\
\hline $7 / 2 / 1$ & $7.75 \mathrm{E}-4$ & 2.07E-03 & $6.28 \mathrm{E}+6$ & $1.247 \pm 0.006 E+2$ & $1.71 \pm 0.11 E+6$ & $1.12 \mathrm{E}+04$ & $1.74 \mathrm{E}+01$ \\
\hline $7 / 2 / 0$ & $4.09 \mathrm{E}-5$ & $8.26 \mathrm{E}-02$ & $3.76 \mathrm{E}+6$ & $9.36 \pm 0.62 E+0$ & $1.87 \pm 0.12 E+3$ & $1.52 \mathrm{E}+05$ & $1.24 \mathrm{E}+03$ \\
\hline $8 / 2 / 7$ & $7.27 \mathrm{E}-2$ & $1.60 \mathrm{E}-02$ & $3.76 \mathrm{E}+6$ & $8.548 \pm 0.09 E+5$ & $3.94 \pm 0.14 E+6$ & $1.64 \mathrm{E}+00$ & $5.59 \mathrm{E}-01$ \\
\hline $8 / 2 / 5$ & $7.79 E-2$ & $9.74 \mathrm{E}-03$ & $3.76 \mathrm{E}+6$ & $1.11 \pm 0.007 E+5$ & $3.45 \pm 0.1 \mathrm{E}+6$ & $1.26 \mathrm{E}+01$ & $6.41 \mathrm{E}-01$ \\
\hline $8 / 2 / 3$ & $4.65 \mathrm{E}+0$ & $7.65 \mathrm{E}-02$ & $3.81 \mathrm{E}+6$ & $2.281 \pm 0.018 \mathrm{E}+4$ & $3.768 \pm 0.047 E+6$ & $6.15 E+01$ & $3.88 \mathrm{E}-01$ \\
\hline $8 / 2 / 2$ & $3.78 \mathrm{E}-2$ & $1.23 \mathrm{E}-01$ & $6.29 \mathrm{E}+6$ & $2.52 \pm 0.032 E+2$ & $1.478 \pm 0.044 E+6$ & $5.56 E+03$ & $1.47 \mathrm{E}+00$ \\
\hline $8 / 2 / 1$ & $5.58 \mathrm{E}-3$ & 1.76E-02 & $6.29 \mathrm{E}+6$ & $1.28 \pm 0.017 E+2$ & $1.51 \pm 0.39 \mathrm{E}+6$ & $1.10 \mathrm{E}+04$ & $3.68 \mathrm{E}+00$ \\
\hline $8 / 2 / 0$ & $4.84 \mathrm{E}-5$ & $2.63 \mathrm{E}-04$ & $6.29 E+6$ & $9.36 \pm 0.62 E+0$ & $9.78 \pm 0.83 E+5$ & $1.52 \mathrm{E}+05$ & $2.70 \mathrm{E}+02$ \\
\hline $9 / 2 / 7$ & $2.01 \mathrm{E}+0$ & $8.88 \mathrm{E}-14$ & $4.04 \pm 0.16 \mathrm{E}+6$ & $2.384 \pm 0.069 \mathrm{E}+6$ & $6.42 \pm 0.3 E+6$ & $5.88 \mathrm{E}-01$ & 2.27E-01 \\
\hline $9 / 2 / 5$ & $3.90 \mathrm{E}+0$ & 2.37E-11 & $3.828 \pm 0.048 E+6$ & $2.529 \pm 0.038 E+5$ & $4.08 \pm 0.056 E+6$ & $5.54 \mathrm{E}+00$ & $3.51 \mathrm{E}-01$ \\
\hline $9 / 2 / 3$ & $1.99 \mathrm{E}+0$ & $9.01 \mathrm{E}-09$ & $4.92 \pm 0.1 \mathrm{E}+6$ & $6.137 \pm 0.024 \mathrm{E}+4$ & $4.99 \pm 0.1 \mathrm{E}+6$ & $2.28 \mathrm{E}+01$ & $2.93 \mathrm{E}-01$ \\
\hline $9 / 2 / 2$ & $4.01 \mathrm{E}+0$ & $6.09 \mathrm{E}-09$ & $5.29 \pm 0.34 \mathrm{E}+6$ & $4.66 \pm 0.89 \mathrm{E}+2$ & $5.29 \pm 0.34 \mathrm{E}+6$ & $3.03 E+03$ & $2.73 \mathrm{E}-01$ \\
\hline $9 / 2 / 1$ & $6.57 \mathrm{E}+1$ & $3.73 \mathrm{E}-09$ & $4.405 \pm 0.084 E+6$ & $1.69 \pm 0.36 \mathrm{E}+2$ & $4.406 \pm 0.084 E+6$ & $8.28 \mathrm{E}+03$ & $3.22 \mathrm{E}-01$ \\
\hline $9 / 2 / 0$ & $6.007 \pm 0.021 \mathrm{E}-4$ & $4.08 \mathrm{E}-06$ & $6.29 E+6$ & $9.36 \pm 0.62 E+0$ & $6.29 E+6$ & $1.52 E+05$ & $1.96 \mathrm{E}+01$ \\
\hline $10 / 2 / 7$ & $1.26 \mathrm{E}+0$ & $2.89 \mathrm{E}-12$ & $3.76 \mathrm{E}+6$ & $1.586 \pm 0.049 \mathrm{E}+6$ & $5.347 \pm 0.098 \mathrm{E}+6$ & $8.84 \mathrm{E}-01$ & $2.75 \mathrm{E}-01$ \\
\hline $10 / 2 / 5$ & $6.83 E+1$ & $5.80 \mathrm{E}-03$ & $3.95 \pm 0.18 \mathrm{E}+6$ & $2.417 \pm 0.034 E+5$ & $4.2 \pm 0.19 \mathrm{E}+6$ & $5.80 \mathrm{E}+00$ & 3.39E-01 \\
\hline $10 / 2 / 3$ & $1.83 \mathrm{E}+0$ & $6.13 \mathrm{E}-03$ & $4.41 \pm 0.14 \mathrm{E}+6$ & $5.054 \pm 0.009 \mathrm{E}+4$ & $4.44 \pm 0.14 E+6$ & $2.77 E+01$ & $3.29 \mathrm{E}-01$ \\
\hline $10 / 2 / 2$ & $3.85 \mathrm{E}-2$ & $3.79 \mathrm{E}-03$ & $4.5 \pm 0.1 \mathrm{E}+6$ & $6.97 \pm 0.058 \mathrm{E}+2$ & $4.1 \pm 0.1 \mathrm{E}+6$ & $2.01 \mathrm{E}+03$ & $8.00 \mathrm{E}-01$ \\
\hline $10 / 2 / 1$ & $8.53 \mathrm{E}+1$ & 4.90E-04 & $3.76 \mathrm{E}+6$ & $3.584 \pm 0.02 E+2$ & $3.76 \mathrm{E}+6$ & $3.91 \mathrm{E}+03$ & $3.76 \mathrm{E}-01$ \\
\hline
\end{tabular}




\begin{tabular}{|c|c|c|c|c|c|c|c|}
\hline $10 / 2 / 0$ & $8.85 \pm 0.25 E-4$ & $1.52 \mathrm{E}-03$ & $3.76 \mathrm{E}+6$ & $9.36 \pm 0.62 E+0$ & $3.76 \mathrm{E}+6$ & $1.52 E+05$ & $1.54 \mathrm{E}+01$ \\
\hline $20 / 2 / 7$ & $1.09 \mathrm{E}+1$ & $2.39 \mathrm{E}-16$ & $5.7 \pm 0.11 \mathrm{E}+6$ & $1.277 \pm 0.024 \mathrm{E}+6$ & $6.98 \pm 0.16 \mathrm{E}+6$ & $1.10 \mathrm{E}+00$ & $2.06 \mathrm{E}-01$ \\
\hline $20 / 2 / 5$ & $5.16 \mathrm{E}+1$ & $6.68 \mathrm{E}-16$ & $6.29 \mathrm{E}+6$ & $2.85 \pm 0.41 E+5$ & $6.579 \pm 0.071 E+6$ & $4.92 \mathrm{E}+00$ & 2.17E-01 \\
\hline $20 / 2 / 3$ & $2.08 \mathrm{E}+0$ & $1.46 \mathrm{E}-16$ & $4.75 \pm 0.11 \mathrm{E}+6$ & $5.201 \pm 0.045 E+4$ & $4.8 \pm 0.11 E+6$ & $2.70 E+01$ & $3.04 \mathrm{E}-01$ \\
\hline $20 / 2 / 2$ & $4.68 \mathrm{E}+0$ & $5.41 \mathrm{E}-16$ & $5.412 \pm 0.072 E+6$ & $8.64 \pm 0.1 \mathrm{E}+2$ & $5.413 \pm 0.072 E+6$ & $1.63 E+03$ & $2.66 \mathrm{E}-01$ \\
\hline $20 / 2 / 1$ & $2.34 \mathrm{E}+0$ & 8.03E-17 & $3.761 \pm 0.012 E+6$ & $3.03 \pm 0.16 E+2$ & $3.762 \pm 0.012 E+6$ & $4.63 E+03$ & $3.83 \mathrm{E}-01$ \\
\hline $20 / 2 / 0$ & $2.038 \pm 0.015 E-3$ & $8.14 \mathrm{E}-17$ & $6.29 E+6$ & $9.36 \pm 0.62 \mathrm{E}+0$ & $6.29 \mathrm{E}+6$ & $1.52 \mathrm{E}+05$ & $6.09 \mathrm{E}+00$ \\
\hline
\end{tabular}




\section{Supplementary Table 16| Spacer Characterisation at $25^{\circ} \mathrm{C}$}

Data used to infer the effect of a spacer sequence between the handhold and the displaced domain. All the relevant characterisation constants are accompanied by the standard error of the mean of individual fittings (Supplementary Note XVI). Data obtained as described in Supplementary Notes II, XIV (to obtain $\boldsymbol{k}_{t}$ ) and XVI. The reactant strands used were all $N$ strands "Incumbent $\mathrm{X} \mathrm{hh/x} \mathrm{sp"}$ excluding 2 nt spacers (Supplementary Table 20) with $T$ strands "Target ( $x$ th)" with toeholds of 0, 2 and $8 \mathrm{nt}$ (Supplementary Table 21) and I strands "Invader $\left(I_{h c}\right)$ ", and to obtain the value of $k_{t}$ "Invader random-5" $\left(I_{w c}\right)$ " (Supplementary Table 22). Reporter strands were "Reporter $1(R)$ " and "Reporter 1 quencher $(Q)$ " (Supplementary Table 23). Legend: Bold: Relevant reaction rate for the system. Yellow highlight: $1^{\text {st }}$ order reaction rates. Green: $k_{b}$ with a negligible standard error. This artefact is produced by the boundaries imposed to $k_{b}$ during its fitting. Red: Rates with a standard error bigger than the $10 \%$ of the mean. Corresponds to long experimental conditions (toehold=0 nt) or systems at the boundary between different kinetic regimes. Red highlight: $k_{t}$ values that were not obtained from direct experimental evidence but the mean of all the systems with the same toehold length. Experiments omitted to reduce the number of extremely slow experiments. The standard error of the mean for each parameter was calculated from the values obtained in individual fittings.

\begin{tabular}{|c|c|c|c|c|c|c|c|}
\hline $\begin{array}{c}\text { System } \\
\mathrm{hh} / \mathrm{sp} / \text { toe }\end{array}$ & $\begin{array}{c}\boldsymbol{k}_{m} \\
\left(s^{-1}\right)\end{array}$ & $\begin{array}{c}k_{d} \\
\left(s^{-1}\right)\end{array}$ & $\begin{array}{c}\boldsymbol{k}_{\boldsymbol{b}} \\
\left(s^{-1} M^{-1}\right)\end{array}$ & $\begin{array}{c}k_{t} \\
\left(s^{-1} M^{-1}\right)\end{array}$ & $\begin{array}{c}2^{\text {nd }} \text { order rate }(k) \\
\text { (eq.3) }\left(s^{-1} M^{-1}\right)\end{array}$ & $\begin{array}{c}\boldsymbol{t}_{1 / 2}^{w c} \\
(\min )\end{array}$ & $\begin{array}{c}\boldsymbol{t}_{1 / 2} \\
(\min )\end{array}$ \\
\hline $0 / 0 / 7$ & 0 & 0 & 0 & $4.39 \pm 0.12 E+6$ & $4.39 \pm 0.12 E+6$ & $2.79 E-01$ & $2.82 \mathrm{E}-01$ \\
\hline $0 / 0 / 2$ & 0 & 0 & 0 & $1.99 \pm 0.1 \mathrm{E}+2$ & $1.99 \pm 0.1 \mathrm{E}+2$ & $7.52 E+03$ & $7.51 E+03$ \\
\hline $0 / 1 / 7$ & 0 & 0 & 0 & $3.43 \pm 0.12 E+6$ & $3.43 \pm 0.12 E+6$ & $4.20 \mathrm{E}-01$ & 4.23E-01 \\
\hline $0 / 1 / 2$ & 0 & 0 & 0 & $1.79 \pm 0.029 E+2$ & $1.79 \pm 0.029 E+2$ & $7.48 \mathrm{E}+03$ & $7.47 E+03$ \\
\hline $0 / 1 / 0$ & 0 & 0 & 0 & $1.041 \pm 0.067 E+1$ & $1.041 \pm 0.067 \mathrm{E}+1$ & $3.78 \mathrm{E}+05$ & - \\
\hline $0 / 3 / 7$ & 0 & 0 & 0 & $2.274 \pm 0.057 E+6$ & $2.274 \pm 0.057 \mathrm{E}+6$ & $5.74 \mathrm{E}-01$ & 5.77E-01 \\
\hline $0 / 3 / 2$ & 0 & 0 & 0 & $1.37 \pm 0.12 E+2$ & $1.37 \pm 0.12 E+2$ & $9.08 E+03$ & $9.07 E+03$ \\
\hline $0 / 5 / 7$ & 0 & 0 & 0 & $5.503 \pm 0.095 E+5$ & $5.503 \pm 0.095 E+5$ & $2.34 \mathrm{E}+00$ & $2.35 E+00$ \\
\hline $0 / 5 / 2$ & 0 & 0 & 0 & $1.407 \pm 0.094 \mathrm{E}+2$ & $1.407 \pm 0.094 \mathrm{E}+2$ & $9.16 \mathrm{E}+03$ & $9.15 E+03$ \\
\hline $0 / 8 / 7$ & 0 & 0 & 0 & $3.25 \pm 0.11 E+5$ & $3.25 \pm 0.11 E+5$ & $3.83 E+00$ & $3.83 E+00$ \\
\hline $0 / 8 / 2$ & 0 & 0 & 0 & $1.093 \pm 0.068 \mathrm{E}+2$ & $1.093 \pm 0.068 \mathrm{E}+2$ & $1.32 \mathrm{E}+04$ & $1.32 \mathrm{E}+04$ \\
\hline $5 / 0 / 7$ & $1.46 \mathrm{E}-1$ & $1.34 \mathrm{E}+01$ & $6.25 E+6$ & $3.09 \pm 0.068 E+5$ & $3.76 \pm 0.17 E+5$ & $4.54 \mathrm{E}+00$ & $3.76 \mathrm{E}+00$ \\
\hline $5 / 0 / 2$ & $1.98 \mathrm{E}-2$ & $2.11 \mathrm{E}+01$ & $6.29 E+6$ & $3.112 \pm 0.043 E+2$ & $6.21 \pm 0.096 \mathrm{E}+3$ & $4.55 E+03$ & $2.27 E+02$ \\
\hline $5 / 0 / 0$ & $1.31 \mathrm{E}-4$ & $7.67 E+00$ & $5.73 E+6$ & $9.36 \pm 0.62 E+0$ & $1.069 \pm 0.048 \mathrm{E}+2$ & $1.52 \mathrm{E}+05$ & $1.33 E+04$ \\
\hline $5 / 1 / 7$ & $8.91 \mathrm{E}-2$ & $2.11 \mathrm{E}+00$ & $3.76 E+6$ & $4.764 \pm 0.07 E+5$ & $6.29 \pm 0.17 E+5$ & $2.94 \mathrm{E}+00$ & $2.29 \mathrm{E}+00$ \\
\hline $5 / 1 / 2$ & $2.28 \mathrm{E}-1$ & $7.80 E+02$ & $5.12 \mathrm{E}+6$ & $2.758 \pm 0.028 \mathrm{E}+2$ & $1.774 \pm 0.023 E+3$ & $5.04 \mathrm{E}+03$ & $7.90 E+02$ \\
\hline $5 / 1 / 0$ & $1.82 \mathrm{E}-6$ & $2.57 E+00$ & $4.51 \mathrm{E}+6$ & $9.36 \pm 0.62 E+0$ & $1.24 \pm 0.13 E+1$ & $1.52 \mathrm{E}+05$ & $1.16 \mathrm{E}+05$ \\
\hline $5 / 3 / 7$ & $3.27 \mathrm{E}-1$ & $2.63 E+00$ & $4.16 \mathrm{E}+6$ & $9.13 \pm 0.16 E+5$ & $1.373 \pm 0.054 \mathrm{E}+6$ & $1.54 \mathrm{E}+00$ & $1.05 E+00$ \\
\hline $5 / 3 / 2$ & $1.19 \mathrm{E}-3$ & $9.16 \mathrm{E}+00$ & $4.03 E+6$ & $3.855 \pm 0.029 E+2$ & $9.12 \pm 0.11 E+2$ & $3.62 E+03$ & $1.55 E+03$ \\
\hline $5 / 3 / 0$ & $5.96 \mathrm{E}-7$ & $9.56 \mathrm{E}+00$ & $3.76 \mathrm{E}+6$ & $9.36 \pm 0.62 E+0$ & $9.5 \pm 1.4 \mathrm{E}+0$ & $1.52 \mathrm{E}+05$ & $1.49 \mathrm{E}+05$ \\
\hline $5 / 5 / 7$ & $2.12 \mathrm{E}-1$ & $6 \mathrm{E}+00$ & $3.76 \mathrm{E}+6$ & $3.64 \pm 0.14 \mathrm{E}+5$ & $4.92 \pm 0.19 \mathrm{E}+5$ & $3.85 E+00$ & $2.88 \mathrm{E}+00$ \\
\hline $5 / 5 / 2$ & $1.80 \mathrm{E}-4$ & $7.62 E+00$ & $6.22 E+6$ & $2.66 \pm 0.025 E+2$ & $4.14 \pm 0.11 E+2$ & $5.27 E+03$ & $3.43 E+03$ \\
\hline $5 / 5 / 0$ & $1.76 \mathrm{E}-9$ & $0.14 \mathrm{E}+00$ & $6.16 \mathrm{E}+6$ & $9.36 \pm 0.62 E+0$ & $9.33 \pm 0.58 \mathrm{E}+0$ & $1.52 \mathrm{E}+05$ & $1.86 \mathrm{E}+05$ \\
\hline $5 / 8 / 7$ & $2.07 \mathrm{E}-1$ & $2.59 E+00$ & $5.40 E+6$ & $4.23 \pm 0.11 E+5$ & $8.22 \pm 0.49 E+5$ & $3.32 E+00$ & $1.76 \mathrm{E}+00$ \\
\hline $5 / 8 / 2$ & $1.78 \mathrm{E}-4$ & $4.7 \mathrm{E}+00$ & $6.29 E+6$ & $1.46 \pm 0.12 E+2$ & $4.05 \pm 0.23 E+2$ & $8.43 E+03$ & $3.53 E+03$ \\
\hline $5 / 8 / 0$ & $6.19 \mathrm{E}-7$ & $2.79 E+00$ & $6.18 \mathrm{E}+6$ & $9.36 \pm 0.62 E+0$ & $1.062 \pm 0.058 \mathrm{E}+1$ & $1.52 \mathrm{E}+05$ & $1.36 \mathrm{E}+05$ \\
\hline $20 / 0 / 7$ & $1.46 \mathrm{E}+1$ & $1.04 \mathrm{E}-20$ & $6.29 \pm 0.11 E+6$ & $6.284 \pm 0.085 E+5$ & $6.92 \pm 0.12 E+6$ & $2.23 E+00$ & $2.08 \mathrm{E}-01$ \\
\hline $20 / 0 / 2$ & $1.03 E+1$ & $6.59 \mathrm{E}-16$ & $3.76 \mathrm{E}+6$ & $1.43 \pm 0.044 E+3$ & $3.76 \mathrm{E}+6$ & $1.02 \mathrm{E}+03$ & $3.78 \mathrm{E}-01$ \\
\hline $20 / 0 / 0$ & $4.015 \pm 0.033 E-4$ & $3.81 \mathrm{E}-16$ & $6.29 \mathrm{E}+6$ & $9.36 \pm 0.62 E+0$ & $6.29 E+6$ & $1.52 \mathrm{E}+05$ & $2.92 \mathrm{E}+01$ \\
\hline $20 / 1 / 7$ & $2.67 \mathrm{E}+0$ & $3.01 E-16$ & $6.29 \mathrm{E}+6$ & $1.34 \pm 0.02 E+6$ & $7.634 \pm 0.02 E+6$ & $1.05 \mathrm{E}+00$ & 1.92E-01 \\
\hline $20 / 1 / 2$ & $2.02 E+0$ & $3.72 \mathrm{E}-16$ & $5.41 \pm 0.13 E+6$ & $1.436 \pm 0.012 E+3$ & $5.41 \pm 0.13 E+6$ & $9.81 E+02$ & 2.71E-01 \\
\hline $20 / 1 / 0$ & $2.62 \pm 0.11 E-3$ & $1.17 \mathrm{E}-16$ & $6.29 E+6$ & $9.36 \pm 0.62 E+0$ & $6.29 E+6$ & $1.52 \mathrm{E}+05$ & $4.84 \mathrm{E}+00$ \\
\hline $20 / 3 / 7$ & $1.39 \mathrm{E}+0$ & $9.14 \mathrm{E}-17$ & $4.8 \pm 0.43 E+6$ & $1.417 \pm 0.063 E+6$ & $6.21 \pm 0.49 E+6$ & $9.89 \mathrm{E}-01$ & $2.38 \mathrm{E}-01$ \\
\hline $20 / 3 / 2$ & $1.66 \mathrm{E}+1$ & $4.1 \mathrm{E}-16$ & $4.672 \pm 0.089 \mathrm{E}+6$ & $1.36 \pm 0.014 E+3$ & $4.673 \pm 0.089 E+6$ & $1.03 E+03$ & 3.04E-01 \\
\hline $20 / 3 / 0$ & $1.28 \pm 0.02 E-3$ & $1.27 \mathrm{E}-16$ & $5.46 \mathrm{E}+6$ & $9.36 \pm 0.62 E+0$ & $5.46 \mathrm{E}+6$ & $1.52 \mathrm{E}+05$ & $9.50 E+00$ \\
\hline $20 / 5 / 7$ & $5.48 \mathrm{E}-1$ & $1.69 \mathrm{E}-16$ & $3.761 \pm 0.032 \mathrm{E}+6$ & $5.291 \pm 0.033 \mathrm{E}+5$ & $4.29 \pm 0.035 E+6$ & $2.65 \mathrm{E}+00$ & $3.58 \mathrm{E}-01$ \\
\hline $20 / 5 / 2$ & 1.27E-1 & $2.58 \mathrm{E}-16$ & $4.52 \pm 0.26 \mathrm{E}+6$ & $7.387 \pm 0.033 E+2$ & $4.53 \pm 0.26 \mathrm{E}+6$ & $1.90 \mathrm{E}+03$ & 4.62E-01 \\
\hline $20 / 5 / 0$ & $6.191 \pm 0.028 E-4$ & $1.67 \mathrm{E}-16$ & $3.96 \mathrm{E}+6$ & $9.41 \pm 0.73 E+0$ & $3.96 \mathrm{E}+6$ & $1.52 \mathrm{E}+05$ & $1.93 E+01$ \\
\hline $20 / 8 / 7$ & 1.74E+1 & $2.65 \mathrm{E}-16$ & $4.047 \pm 0.045 E+6$ & $7.752 \pm 0.044 E+5$ & $4.822 \pm 0.049 \mathrm{E}+6$ & $1.81 \mathrm{E}+00$ & 2.95E-01 \\
\hline $20 / 8 / 2$ & $1.68 \mathrm{E}-1$ & $5.73 \mathrm{E}-16$ & $4.2 \pm 0.13 E+6$ & $6.429 \pm 0.045 E+2$ & $4.21 \pm 0.13 E+6$ & $2.21 E+03$ & 4.49E-01 \\
\hline $20 / 8 / 0$ & $5.686 \pm 0.03 E-4$ & $2.17 E-16$ & $3.76 \mathrm{E}+6$ & $9.36 \pm 0.62 E+0$ & $3.76 \mathrm{E}+6$ & $1.52 \mathrm{E}+05$ & $2.10 E+01$ \\
\hline
\end{tabular}




\section{Supplementary Table 17| Handhold Characterisation at $37^{\circ} \mathrm{C}$}

All the relevant characterisation constants are accompanied by the standard error of the mean between all the obtained fluorescence traces for that reaction system. Data obtained as described in Supplementary Notes II, XIV (to obtain $\boldsymbol{k}_{t}$ ) and XVI. The reactant strands used were $N$ strands "Incumbent $\mathrm{x} \mathrm{hh/x} \mathrm{sp"} \mathrm{with} \mathrm{spacers} 0,2$ and 8 (Supplementary Table 20) with $T$ strands "Target (x th)" with toeholds of 0, 2 and $7 \mathrm{nt}$ (Supplementary Table 21) and I strands "Invader $\left(I_{h c}\right)$ ", and to obtain the value of $k_{t}$ "Invader random-5' $\left(I_{w c}\right)$ " (Supplementary Table 22). Reporter strands were "Reporter $1(R)$ " and "Reporter 1 quencher $(Q)$ " (Supplementary Table 23). Legend: Bold: Relevant reaction rate for the system. Yellow highlight: $1^{\text {st }}$ order reaction rates. Red: Rates with a standard error bigger than the $10 \%$ of the mean. Corresponds with long experimental conditions (toehold $=0 \mathrm{nt}$ ) or systems at the boundary between different kinetic regimes. Red highlight: $k_{t}$ values that were not obtained from direct experimental evidence. Their value was estimated from the $k_{t}$ for a toehold $=0 \mathrm{nt}$ at $25^{\circ} \mathrm{C}$. The standard error of the mean for each parameter was calculated from the values obtained in individual fittings.

\begin{tabular}{|c|c|c|c|c|c|c|c|}
\hline $\begin{array}{c}\text { System } \\
\mathrm{hh} / \mathrm{sp} / \text { toe }\end{array}$ & $\begin{array}{c}k_{m} \\
\left(s^{-1}\right)\end{array}$ & $\begin{array}{c}k_{d} \\
\left(s^{-1}\right)\end{array}$ & $\begin{array}{c}\boldsymbol{k}_{\boldsymbol{b}} \\
\left(s^{-1} M^{-1}\right)\end{array}$ & $\begin{array}{c}k_{t} \\
\left(s^{-1} M^{-1}\right)\end{array}$ & $\begin{array}{c}2^{\text {nd }} \text { order rate }(k) \\
\text { (eq.3) }\left(s^{-1} M^{-1}\right)\end{array}$ & $\begin{array}{c}\boldsymbol{t}_{1 / 2}^{W C} \\
(\min )\end{array}$ & $\begin{array}{c}\boldsymbol{t}_{1 / 2} \\
(\min )\end{array}$ \\
\hline $0 / 0 / 7$ & 0 & 0 & 0 & $5.14 \pm 0.11 E+6$ & $5.14 \pm 0.11 \mathrm{E}+6$ & $2.38 \mathrm{E}-01$ & $2.41 \mathrm{E}-1$ \\
\hline $0 / 0 / 2$ & 0 & 0 & 0 & $4.33 \pm 0.2 \mathrm{E}+2$ & $4.24 \pm 0.19 E+2$ & $3.63 E+03$ & $3.63 E+3$ \\
\hline $0 / 8 / 7$ & 0 & 0 & 0 & $4.27 \pm 0.053 E+5$ & $4.27 \pm 0.053 E+5$ & $2.53 \mathrm{E}+00$ & $2.53 \mathrm{E}+0$ \\
\hline $0 / 8 / 2$ & 0 & 0 & 0 & $2.71 \pm 0.16 E+2$ & $2.65 \pm 0.16 \mathrm{E}+2$ & $5.43 E+03$ & $5.42 \mathrm{E}+3$ \\
\hline $5 / 0 / 7$ & $2.3 \mathrm{E}+0$ & $3.37 \mathrm{E}+02$ & $8.34 \mathrm{E}+06$ & $3.746 \pm 0.048 E+5$ & $4.312 \pm 0.127 \mathrm{E}+5$ & $3.74 \mathrm{E}+00$ & $3.26 \mathrm{E}+0$ \\
\hline $5 / 0 / 2$ & $3.61 \mathrm{E}-2$ & $1.82 \mathrm{E}+02$ & $7.66 \mathrm{E}+06$ & $4.32 \pm 0.17 E+2$ & $1.955 \pm 0.187 E+3$ & $3.31 E+03$ & $7.18 \mathrm{E}+2$ \\
\hline $5 / 0 / 0$ & $1.22 \mathrm{E}-3$ & $1.28 \mathrm{E}+02$ & $5.73 E+06$ & $9.36 \pm 0.62 \mathrm{E}+0$ & $6.419 \pm 0.073 \mathrm{E}+1$ & $1.52 \mathrm{E}+05$ & $2.18 \mathrm{E}+4$ \\
\hline $5 / 8 / 7$ & $1.82 \mathrm{E}+0$ & $1.19 \mathrm{E}+02$ & $1.04 \mathrm{E}+07$ & $4.915 \pm 0.08 \mathrm{E}+5$ & $6.472 \pm 0.133 E+5$ & $2.85 \mathrm{E}+00$ & $2.17 \mathrm{E}+0$ \\
\hline $5 / 8 / 2$ & $2.85 \mathrm{E}-4$ & $3.59 \mathrm{E}+01$ & $5.57 \mathrm{E}+06$ & $3.4 \pm 0.19 \mathrm{E}+2$ & $3.841 \pm 0.284 \mathrm{E}+2$ & $4.21 E+03$ & $3.66 \mathrm{E}+3$ \\
\hline $5 / 8 / 0$ & $4.36 \mathrm{E}-5$ & $4.80 \mathrm{E}+01$ & 7.97E+06 & $9.36 \pm 0.62 E+0$ & $1.661 \pm 0.123 E+1$ & $1.52 \mathrm{E}+05$ & $8.45 E+4$ \\
\hline $20 / 0 / 7$ & $1.24 \mathrm{E}+1$ & $4.36 \mathrm{E}-10$ & $1.459 \pm 0.003 E+7$ & $6.424 \pm 0.083 E+5$ & $1.5229 \pm 0.003 E+7$ & $2.18 \mathrm{E}+00$ & $9.82 \mathrm{E}-2$ \\
\hline $20 / 0 / 2$ & 7.93E+0 & $1.04 \mathrm{E}-10$ & $1.054 \pm 0.025 \mathrm{E}+7$ & $5.33 \pm 1 E+2$ & $1.054 \pm 0.025 \mathrm{E}+7$ & $2.76 \mathrm{E}+03$ & $1.40 \mathrm{E}-1$ \\
\hline $20 / 0 / 0$ & $6.456 \pm 0.081 E-4$ & $1.18 \mathrm{E}-11$ & 1.19E+07 & $9.36 \pm 0.62 E+0$ & 1.19E+7 & $1.52 \mathrm{E}+05$ & $1.81 \mathrm{E}+1$ \\
\hline $20 / 8 / 7$ & $6.37 \mathrm{E}+0$ & $6.98 \mathrm{E}-11$ & $5.57 \pm 0.27 E+6$ & $6.187 \pm 0.078 E+5$ & $6.19 \pm 0.28 E+6$ & $2.27 E+00$ & $2.32 \mathrm{E}-1$ \\
\hline $20 / 8 / 2$ & $1.31 \mathrm{E}+1$ & $1.52 \mathrm{E}-06$ & $8.59 \pm 0.75 E+6$ & $3.77 \pm 0.4 E+2$ & $8.59 \pm 0.75 E+6$ & $3.79 E+03$ & $1.68 \mathrm{E}-1$ \\
\hline $20 / 8 / 0$ & $1.323 \pm 0.008 E-3$ & $8.75 \mathrm{E}-12$ & $1.44 \mathrm{E}+07$ & $9.36 \pm 0.62 E+0$ & $1.44 \mathrm{E}+7$ & $1.52 \mathrm{E}+05$ & $8.9 \mathrm{E}+0$ \\
\hline $4 / 2 / 0$ & $3.58 \mathrm{E}-4$ & $1.95 \mathrm{E}+02$ & $8.67 \mathrm{E}+06$ & $9.36 \pm 0.62 E+0$ & $2.527 \pm 0.104 \mathrm{E}+1$ & $5.58 \mathrm{E}+04$ & $5.55 \mathrm{E}+4$ \\
\hline $5 / 2 / 0$ & $1.39 \mathrm{E}-4$ & $5.01 \mathrm{E}+01$ & $7.66 \mathrm{E}+06$ & $9.36 \pm 0.62 E+0$ & $3.798 \pm 0.11 E+1$ & $3.71 E+04$ & $3.70 \mathrm{E}+4$ \\
\hline $6 / 2 / 0$ & $1.41 \mathrm{E}-4$ & $9.13 \mathrm{E}+00$ & $6.41 \mathrm{E}+06$ & $9.36 \pm 0.62 E+0$ & $1.08 \pm 0.11 E+2$ & $1.31 \mathrm{E}+04$ & $1.31 \mathrm{E}+4$ \\
\hline $7 / 2 / 0$ & $1.78 \mathrm{E}-4$ & $3.80 E+00$ & $7.07 E+06$ & $9.36 \pm 0.62 E+0$ & $3.395 \pm 0.099 \mathrm{E}+2$ & $4.35 \mathrm{E}+03$ & $4.25 E+3$ \\
\hline $8 / 2 / 0$ & $2.80 \mathrm{E}-4$ & $1.06 \mathrm{E}+00$ & $5.57 \mathrm{E}+06$ & $9.36 \pm 0.62 E+0$ & $1.486 \pm 0.031 E+3$ & $1.02 \mathrm{E}+03$ & $1.02 \mathrm{E}+3$ \\
\hline $9 / 2 / 0$ & $1.85 \pm 0.17 E-4$ & $1.37 \mathrm{E}-02$ & $9.02 E+06$ & $9.36 \pm 0.62 E+0$ & $1.2 \mathrm{E}+5$ & $8.20 E+01$ & $9.73 E+1$ \\
\hline $10 / 2 / 0$ & $5.65 \pm 0.36 \mathrm{E}-4$ & $1.53 \mathrm{E}-03$ & $9.60 \mathrm{E}+06$ & $9.36 \pm 0.62 E+0$ & $2.59 E+6$ & $2.27 \mathrm{E}+01$ & $2.27 E+1$ \\
\hline $20 / 2 / 0$ & $1.78 \pm 0.28 \mathrm{E}-3$ & $1.60 \mathrm{E}-11$ & $5.57 E+06$ & $9.36 \pm 0.62 E+0$ & $5.57 \mathrm{E}+6$ & $7.02 E+00$ & $6.96 \mathrm{E}+0$ \\
\hline
\end{tabular}




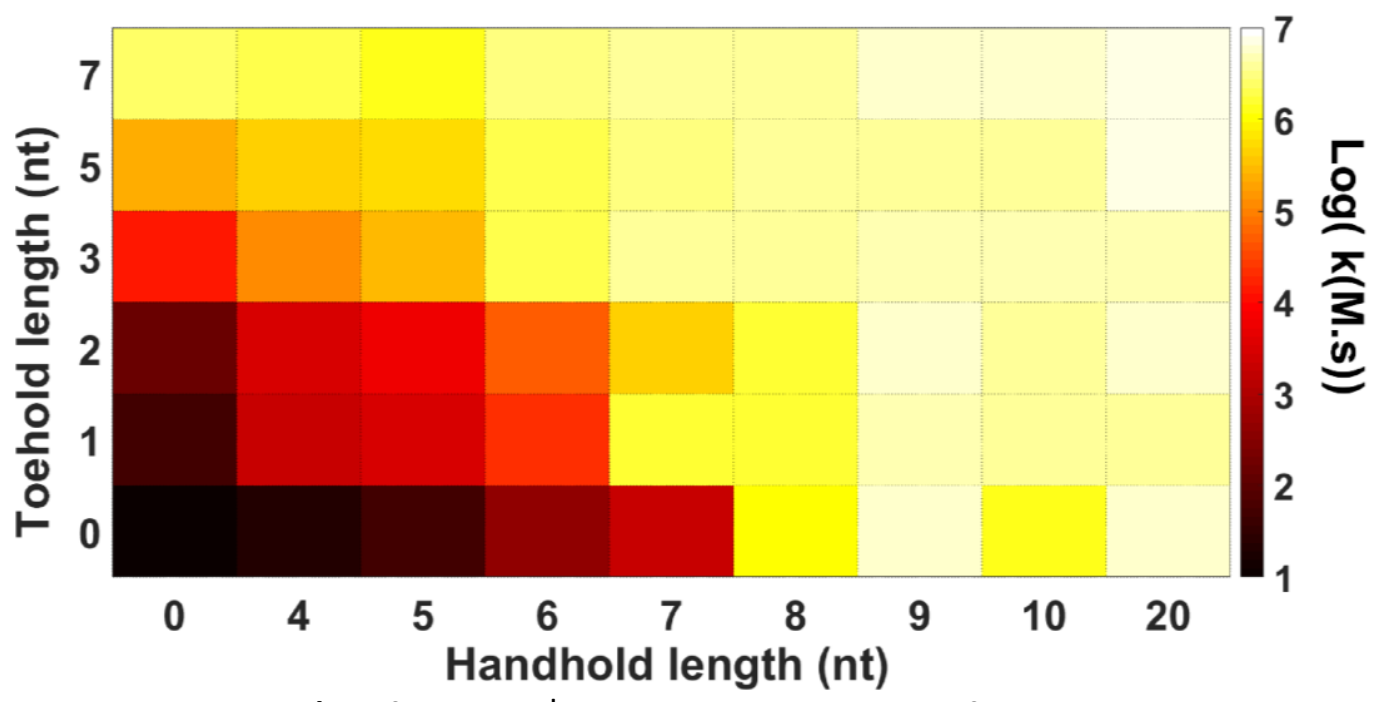

Supplementary Figure 18 Inferred $2^{\text {nd }}$ order rate constants for handhold-mediated strand displacement.

$k$ values collected in Supplementary Table 15. Reaction rates for toeholds $=0$ nt do not correspond with their real $1^{\text {st }}$ order reaction rate.

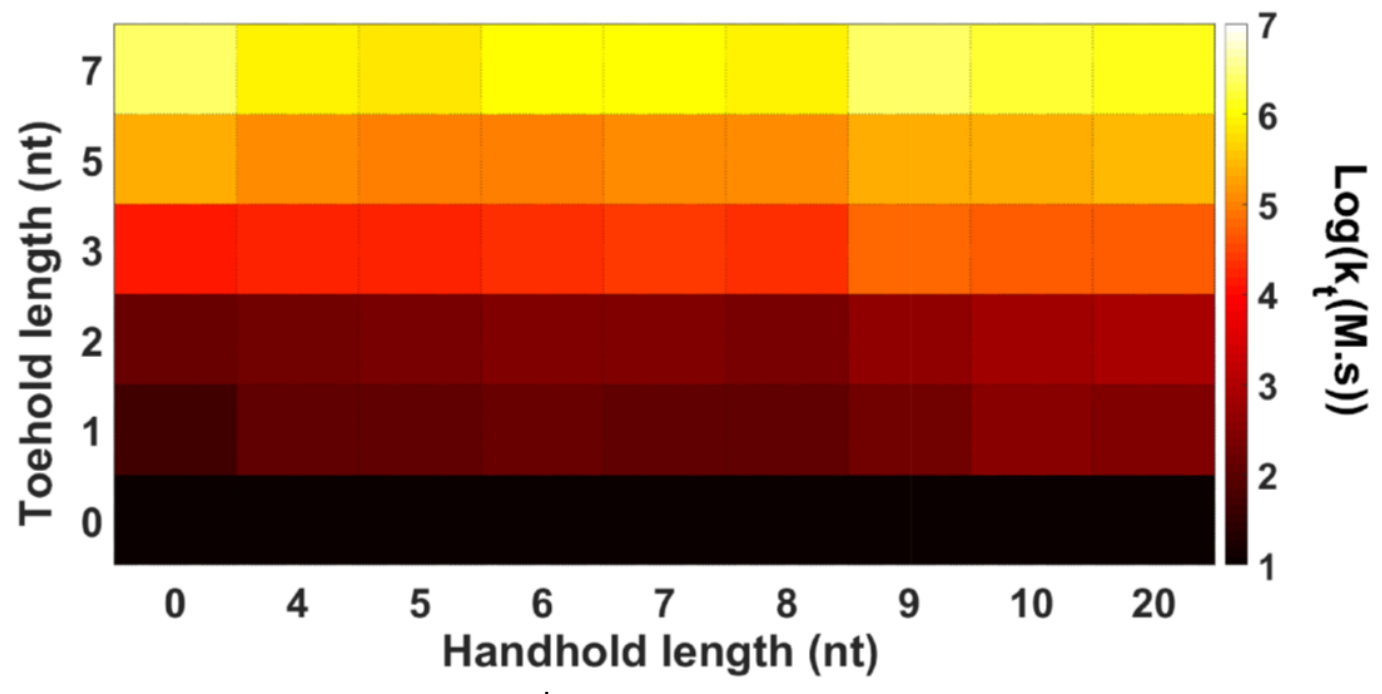

Supplementary Figure 19| Inferred $2^{\text {nd }}$ order rate constants for I-strands without handhold complementarity.

The reaction rates are the ones of a toehold mediated strand displacement $\left(k_{t}\right.$, Supplementary Table 15). Handhold presence in $N$ produces negligible rate changes for strand displacement reactions when $\mathrm{l}$-strands lack handhold-complementarity. 


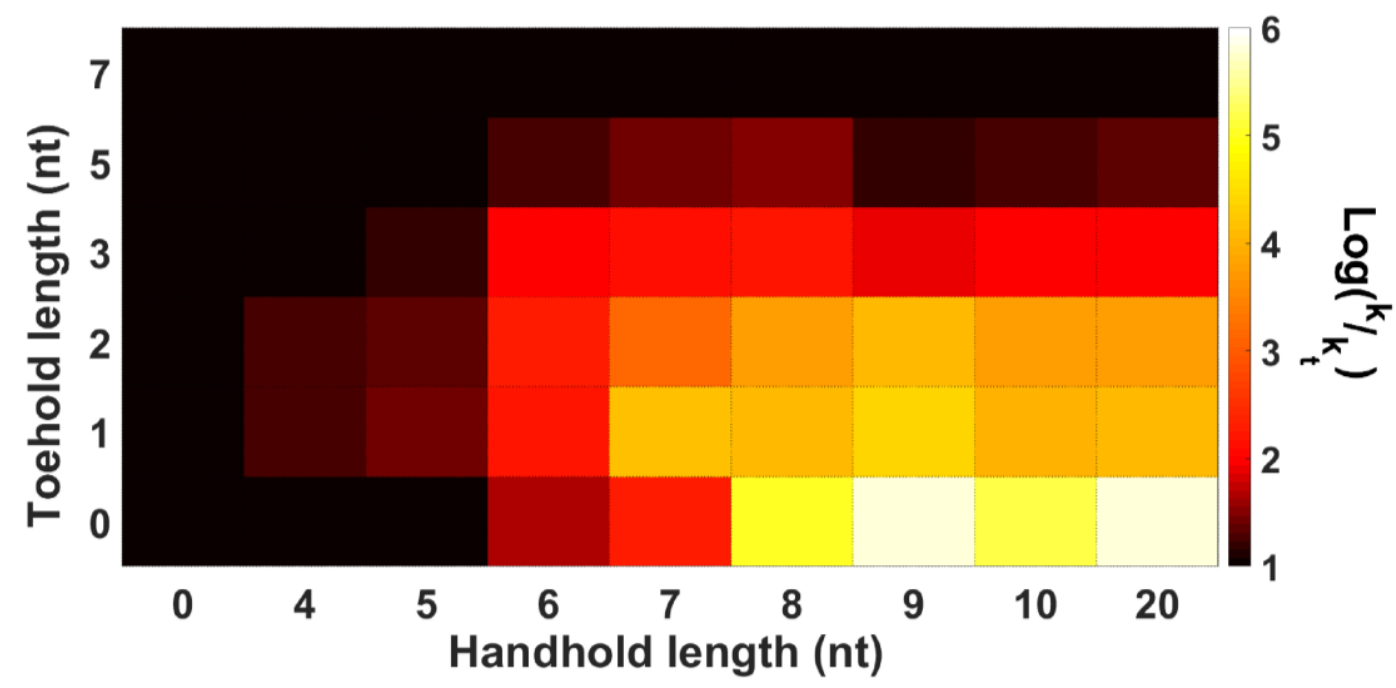

Supplementary Figure 20| Comparison between $\boldsymbol{k}$ and $\boldsymbol{k}_{\boldsymbol{t}}$

Our inferred $2^{\text {nd }}$ order rate constants suggest that HMSD can increase displacement rate by up to 5 orders of magnitude compared to displacement triggered with 1 -strands without handholdcomplementarity. The reaction rate increment obtained by the addition of handholdcomplementarity is sensibly higher in the regime of toeholds below $3 \mathrm{nt}$. 


\section{Supplementary Note XVII: Fitting of the kinetic analysis of handhold detachment}

$R_{2} Q_{2}$ controls shown in Supplementary Note IX proved that the presence of strand $T$ in the solution triggered $R_{2} Q_{2}$ associative toehold at a slow rate, except when free in solution as a product $I T$. Due to the high reaction rate of $R_{2} Q_{2}$ with $I T$, the slow-rate leak was ignored when fitting the kinetics of $R_{2} Q_{2}$. The obtained trajectories were fitted to obtain the value of $K_{\text {eq }}$ (equation 6 ). Each trajectory was fit individually to the ODE describing equation $\mathbf{5}$ :

$$
\frac{\mathrm{d}\left[\mathrm{R}_{2} I T\right]}{\mathrm{dt}}=k_{\mathrm{r} 2}\left[R_{2} Q\right][I T],
$$

With constraints:

$$
[I T]=\frac{-\left(K_{2} Q_{2}\right]=\left[R_{2} Q_{2}\right]_{0}-\left[R_{2} I T\right],(29)}{2}
$$

Supplementary equation $\mathbf{3 0}$ describes free $I T$ assuming an instantaneous equilibrium between $N / I T$ and $I T+N$. The detailed derivation of Supplementary equation $\mathbf{3 0}$ from equation $\mathbf{6}$ of the main text is included in the Supplementary Note XIX. The values of $k_{r 2}$ were obtained from individually fitting Supplementary equations 24 and 25 to the kinetics of a system in the absence of handhold (Supplementary Figure 21)

For each system, the model was fitted to individual fluorescence traces to obtain the value of $K_{\text {eq, }}\left[I_{0}\right.$ and $t_{0}$. $K_{\text {eq_init }}$ was set arbitrarily to $5 \cdot 10^{-9} \mathrm{nM}$. For the obtained $K_{\text {eq }}$, the percentage of expected detached $[I T]$ in the equilibrium was calculated with Supplementary equation $\mathbf{3 0}$ for $[N]_{0} ;[]_{0}=10 \mathrm{nM}$ and $\left[R_{2} / T\right]=0 \mathrm{nM}$. Fitted $K_{\text {eq }}$ and the expected percentage of detached [IT] are collected in Supplementary Table 18. Results for the control without $R Q$ reporter are collected in Supplementary Table 19.

Supplementary Table 18| Fitting results from the kinetic analysis of handhold detachment Data obtained as described in Supplementary Notes III and XVII. The reactant strands used were all $N$ strands "Incumbent $\mathrm{x}$ hh/2 sp" (Supplementary Table 20) with "Target(3 th)- $R_{2} Q_{2}$ trigger" (Supplementary Table 21) and "Invader ( $\left.I_{h c}\right)$ " (Supplementary Table 22). Reporter strands were "Reporter $1(R)$ ", "Reporter 1 quencher $(Q)$ ", " $\operatorname{Rep}_{\mathrm{A}}\left(R_{2}\right)$ " and "Rep $p_{\mathrm{A}}$ quencher $\left(Q_{2}\right)$ "(Supplementary Table 23).

\begin{tabular}{c|c|c}
$\begin{array}{c}\text { Condition } \\
\text { (handhold/spacer) }\end{array}$ & $\begin{array}{c}\boldsymbol{K}_{\text {eq }} \\
(\boldsymbol{M})\end{array}$ & $\begin{array}{c}\text { \% of detached } \\
\text { displacement products }\end{array}$ \\
\hline $\mathbf{4 / 2}$ & $1.27 \mathrm{E}-1$ & 99.99 \\
$\mathbf{5 / 2}$ & $1.19 \mathrm{E}-1$ & 99.99 \\
$\mathbf{6 / 2}$ & $9 \mathrm{E}-1$ & 99.99 \\
$\mathbf{7 / 2}$ & $1.01 \mathrm{E}-1$ & 99.99 \\
$\mathbf{8 / 2}$ & $2.1 \mathrm{E}-1$ & 99.99 \\
$\mathbf{9 / 2}$ & $5.6 \mathrm{E}-8$ & $83.3 \pm 2.8$ \\
$\mathbf{1 0 / 2}$ & $3.945 \mathrm{E}-9$ & $45.6 \pm 1.7$ \\
$\mathbf{2 0 / 2}$ & $\sim 0$ & 0
\end{tabular}


Supplementary Table 19| Fitting results from the kinetic analysis of handhold detachment in the absence of reporter $R Q$

Data obtained as described in Supplementary Notes III and XVII. The reactant strands used were $N$ strands "Incumbent $\mathrm{x}$ hh/2 sp" with handholds 7, 8, 9 and 10 nt (Supplementary Table 20) with "Target(3 th)- $R_{2} Q_{2}$ trigger" (Supplementary Table 21) and "Invader ( $\left.I_{h c}\right)$ " (Supplementary Table 22). Reporter strands were " $\operatorname{Rep}_{\mathrm{A}}\left(R_{2}\right)$ " and "Rep $\mathrm{A}_{\mathrm{A}}$ quencher $\left(Q_{2}\right)$ " (Supplementary Table 23). The standard error of the mean for each parameter was calculated from the values obtained in individual fittings.

\begin{tabular}{c|c|c}
$\begin{array}{c}\text { Condition } \\
\text { (handhold/spacer) }\end{array}$ & $\begin{array}{c}\text { Keq } \pm \text { SEM } \\
\text { \% of detached } \\
\text { displacement products }\end{array}$ \\
\hline $\mathbf{7 / 2}$ & $1.01 \mathrm{E}-1$ & 99.99 \\
$\mathbf{8 / 2}$ & $1.77 \mathrm{E}-1$ & 99.99 \\
$\mathbf{9 / 2}$ & $2.381 \pm 0.02 \mathrm{E}-8$ & $75.84 \pm 0.14$ \\
$\mathbf{1 0 / 2}$ & $5.22 \pm 0.23 \mathrm{E}-9$ & $50.68 \pm 0.82$
\end{tabular}

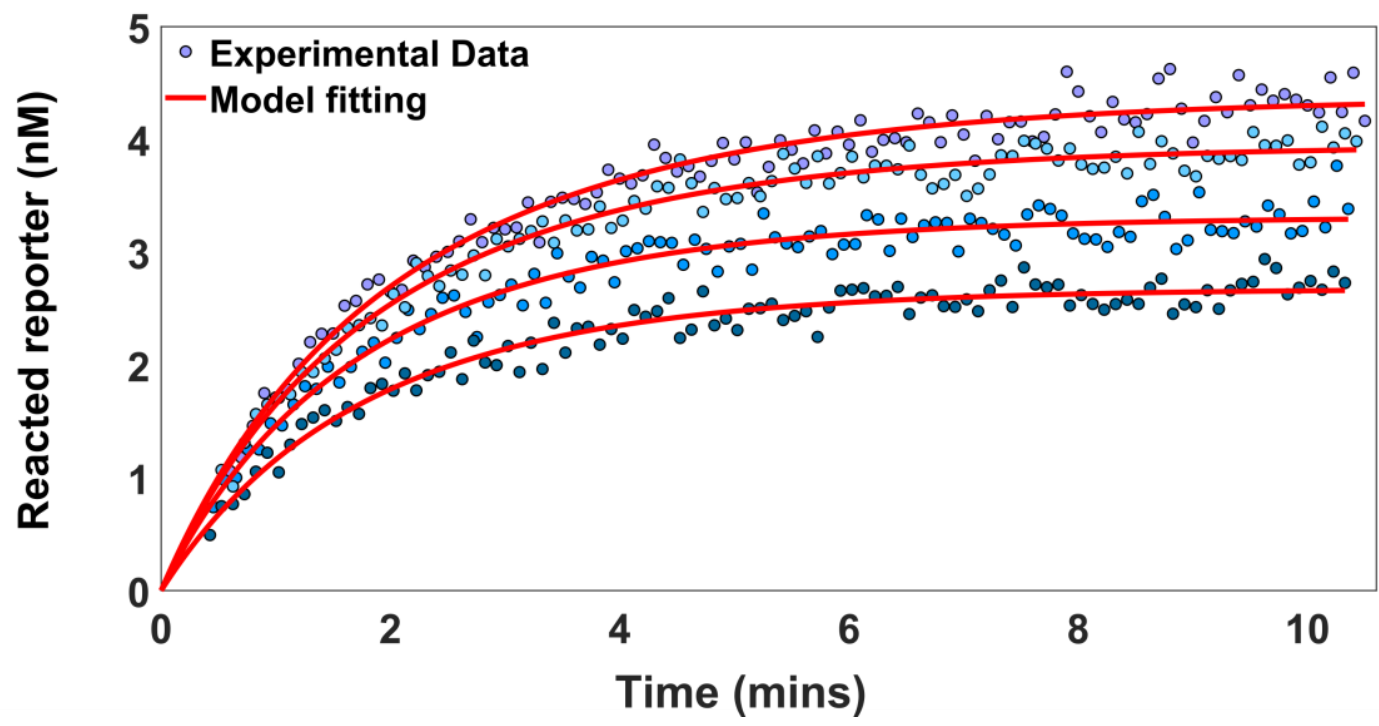

Supplementary Figure 21| Triggering of reporter $R_{2} Q_{2}$ in the absence of handhold.

Processed fluorescence data of the addition of $R_{2} Q_{2}$ (Measurement 4) after the reaction of a system without handhold, a $2 \mathrm{nt}$ spacer, and a $3 \mathrm{nt}$ toehold that has reacted with $I_{\mathrm{hc}}$. Each trajectory was fitted individually with Supplementary equations 28 and 29 to obtain the value of $k_{r 2}$. In the absence of a handhold, all IT was assumed to be detached. The obtained values for $k_{r 2}$ were $6.51,7.14,7.58$ and 7.33.10 $\mathrm{M}^{-1} \mathrm{~s}^{-1}$, which produces a mean of $7.14 \pm 0.23 .10^{5} \mathrm{M}^{-1} \mathrm{~s}^{-1}$. All concentrations are defined for a reaction volume of $200 \mu \mathrm{L}$. 


\section{Supplementary Note XVIII: Derivation of HMSD bimolecular rate}

\section{(equation 4)}

To calculate the effective rate of IT production, we omit the reporter reaction and assume the species $T N / I$ to be in quasi-steady state, since $k_{d}$ and $k_{m}$ are assumed to be large:

$$
\begin{gathered}
\frac{d[T N / I]}{d t}=k_{b}[T N][I]-k_{d}[T N / I]-k_{m}[T N / I]=0 \\
{[T N / I]=\frac{k_{b}}{k_{d}+k_{m}}[T N][I](31)}
\end{gathered}
$$

So, the rate of production of IT for handhold mediated strand displacement is defined:

$$
\frac{d[I T]}{d t}=k_{m}[T N / I]=k_{m} \frac{k_{b}}{k_{d}+k_{m}}[T N][I](32)
$$

Toehold-mediated strand displacement also contributes to the reaction with a rate equal to $k_{t}[T N][I]$. By adding the contribution of handhold and toehold mediated displacements we obtain the final rate:

$$
\frac{d[I T]}{d t}=\left(k_{m} \frac{k_{b}}{k_{d}+k_{m}}+k_{t}\right)[T N][I]=k[T N][I]
$$




\section{Supplementary Note XIX: Derivation of Supplementary equation 30}

From the equilibrium equation (equation 6), it is possible to obtain a boundary for the value of [IT] that depends on the concentrations of the species in the solution. The first step is to substitute $[N]=[N]_{0}-[N / I T]$ and $[N / I T]=[I]_{0}-[I T]-\left[R_{2} / T\right]$ :

$$
\begin{gathered}
K_{e q}=\frac{[N][I T]}{[N / I T]}=\frac{\left([N]_{0}+[I T]+\left[R_{2} / T\right]-[I]_{0}\right)[I T]}{[I]_{0}-[I T]-\left[R_{2} / T\right]}, \quad(34) \\
\left([I]^{0}-[I T]-\left[R_{2} / T\right]\right) K_{e q}=\left([N]_{0}+[I T]+\left[R_{2} / T\right]-[I]_{0}\right)[I T], \\
K_{e q}\left([I]^{0}-\left[R_{2} / T\right]\right)-K_{e q}[I T]=[I T]^{2}+\left([N]_{0}+\left[R_{2} / T\right]-[I]_{0}\right)[I T], \\
0=[I T]^{2}+\left(K_{e q}+[N]_{0}+\left[R_{2} / T\right]-[I]_{0}\right)[I T]+K_{e q}\left(\left[R_{2} / T\right]-[I]_{0}\right)(35),
\end{gathered}
$$

$[I T]$ (Supplementary equation 30 ) is the positive solution of the quadratic equation:

$$
[I T]=\frac{-\left(K_{e q}+[N]_{0}+\left[R_{2} I T\right]-[I]_{0}\right)+\sqrt{\left(K_{e q}+[N]_{0}+\left[R_{2} I T\right]-[I]_{0}\right)^{2}-4 K_{e q}\left(\left[R_{2} I T\right]-[I]_{0}\right)}}{2}
$$




\section{Supplementary Note XX:Sequences}

All sequences used during the experiments are collected in Supplementary Table 20 to 23. Strands are named by their handhold ( $\mathrm{hh}$ ), spacer (sp) and toehold (th) sequences length. The combination of these strands produces the complexes referred during the supplementary as "X/X/X_Temperature". For example, the combination of Incumbent(5hh/2sp) with Target(7nt) reacting at $25^{\circ} \mathrm{C}$, is referred to as the system $5 / 2 / 7 \_25$. The domains of each strand are coded in the same colour as in Figures 1 and 3, specifically:

Grey: $R$ binding domain in $N$ strands and binding domain between $R$ and $Q$ strands.

Orange: $N$ strands domain. Displaced domain during the strand displacement. Same sequence as green.

Green: / strands domain. Same sequence as orange.

Blue: $T$ strands domain. Complementary to orange/green

Gold: Handhold domain of variable length in $N$ strands. Variable 8 nt sequence in reporters. The handhold-complementary sequence in I strands.

Light blue: Toehold domain. Always the same sequence, but with variable length in $T$ strands. $7 \mathrm{nt}$ for $/$ strands and $2 \mathrm{nt}$ for $I_{A / B / C}$ strands

Black: Spacer domains between orange and gold domains in $N$. Hairpins, poly-T spacers and clamps in Rep $\mathrm{p}_{\mathrm{A} / \mathrm{B} / \mathrm{C}} \mathrm{strands}$

Purple: $\operatorname{Rep}_{\mathrm{A} / \mathrm{B} / \mathrm{C}}$ binding domain in $N$ strands and binding domain between $\operatorname{Rep}_{\mathrm{A} / \mathrm{B} / \mathrm{C}}$ and their quenchers. 


\section{Supplementary Table 20|Incumbent strands ( $N$ )}

Incumbent (0 hh/0 sp) Incumbent (0 hh/1 sp) Incumbent $(0 \mathrm{hh} / 2 \mathrm{sp})$ Incumbent $(0 \mathrm{hh} / 3 \mathrm{sp})$ Incumbent $(0 \mathrm{hh} / 5 \mathrm{sp})$ ncumbent $(0 \mathrm{hh} / 8 \mathrm{sp})$ Incumbent (4 hh/2 sp) Incumbent ( $5 \mathrm{hh} / 0 \mathrm{sp})$ Incumbent ( $5 \mathrm{hh} / 1 \mathrm{sp}$ ) (ncumbent (5 hh/2 sp) Incumbent (5 hh/2 sp) Incumbent $(5 \mathrm{hh} / 3 \mathrm{sp})$ Incumbent $(5 \mathrm{hh} / 5 \mathrm{sp})$ Incumbent ( $5 \mathrm{hh} / 8 \mathrm{sp})$ Incumbent (6 hh/2 sp) Incumbent ( $7 \mathrm{hh} / 2 \mathrm{sp})$ Incumbent ( $8 \mathrm{hh} / 2 \mathrm{sp})$ Incumbent $(9 \mathrm{hh} / 2 \mathrm{sp})$ Incumbent $(9 \mathrm{hh} / 2 \mathrm{sp})$ Incumbent $(10 \mathrm{hh} / 2 \mathrm{sp})$ Incumbent $(20 \mathrm{hh} / 0 \mathrm{sp})$ Incumbent (20 hh/1 sp) Incumbent (20 hh/2 sp) Incumbent $(20 \mathrm{hh} / 3 \mathrm{sp})$ Incumbent $(20 \mathrm{hh} / 5 \mathrm{sp})$ Incumbent $(20 \mathrm{hh} / 8 \mathrm{sp})$ Template $A(8 \mathrm{hh} / 2 \mathrm{sp})$ Template $A(8 \mathrm{hh} / 2 \mathrm{sp})$ Template B $(8 \mathrm{hh} / 2 \mathrm{sp})$ Template C ( $8 \mathrm{hh} / 2 \mathrm{sp})$
5' - CCC TAT TAC CCT TGC CGA CCC TAC CTA CCT ACC CTG CG - 3'

$5^{\prime}$ - CCC TAT TAC CCT TGC CGA CCC TAC CTA CCT ACC CTG CGA T - 3'

$5^{\prime}$ - CCC TAT TAC CCT TGC CGA CCC TAC CTA CCT ACC CTG CGA TA - 3'

5' - CCC TAT TAC CCT TGC CGA CCC TAC CTA CCT ACC CTG CGA TAC C - 3'

$5^{\prime}$ - CCC TAT TAC CCT TGC CGA CCC TAC CTA CCT ACC CTG CGA TAC CTA A - 3'

$5^{\prime}$ - CCC TAT TAC CCT TGC CGA CCC TAC CTA CCT ACC CTG CGA TGA TG - $3^{\prime}$

$5^{\prime}$ - CCC TAT TAC CCT TGC CGA CCC TAC CTA CCT ACC CTG CGG ATG T - 3'

$5^{\prime}$ - CCC TAT TAC CCT TGC CGA CCC TAC CTA CCT ACC CTG CGA GAT GT - 3'

$5^{\prime}$ - CCC TAT TAC CCT TGC CGA CCC TAC CTA CCT ACC CTG CGA TGA TGT - 3'

$5^{\prime}$ - CCC TAT TAC CCT TGC CGA CCC TAC CTA CCT ACC CTG CGA TAG ATG T - 3'

$5^{\prime}$ - CCC TAT TAC CCT TGC CGA CCC TAC CTA CCT ACC CTG CGA TAC CGA TGT - 3'

$5^{\prime}$ - CCC TAT TAC CCT TGC CGA CCC TAC CTA CCT ACC CTG CGA TAC CTA AGA TGT - 3'

$5^{\prime}$ - CCC TAT TAC CCT TGC CGA CCC TAC CTA CCT ACC CTG CGA TGA TGT G - 3'

$5^{\prime}$ - CCC TAT TAC CCT TGC CGA CCC TAC CTA CCT ACC CTG CGA TGA TGT GA - 3'

$5^{\prime}$ - CCC TAT TAC CCT TGC CGA CCC TAC CTA CCT ACC CTG CGA TGA TGT GAA - $3^{\prime}$

$5^{\prime}$ - CCC TAT TAC CCT TGC CGA CCC TAC CTA CCT ACC CTG CGA TGA TGT GAA G - 3'

$5^{\prime}$ - CCC TAT TAC CCT TCC CGA CCC TAC CTA CCT ACC CTG CCA TCA TCT GAA GT - 3'

$5^{\prime}$ - CCC TAT TAC CCT TGC CGA CCC TAC CTA CCT ACC CTG CGG ATG TGA AGT GAA TGA TGA G - 3'

$5^{\prime}$ - CCC TAT TAC CCT TGC CGA CCC TAC CTA CCT ACC CTG CGA GAT GTG AAG TGA ATG ATG AG - 3'

$5^{\prime}$ - CCC TAT TAC CCT TGC CGA CCC TAC CTA CCT ACC CTG CGA TGA TGT GAA GTG AAT GAT GAg - 3'

$5^{\prime}$ - CCC TAT TAC CCT TGC CGA CCC TAC CTA CCT ACC CTG CGA TAG ATG TGA AGT GAA TGA TGA G - 3'

5' - CCC TAT TAC CCT TGC CGA CCC TAC CTA CCT ACC CTG CGA TAC CGA TGT GAA GTG AAT GAT GAG - 3'

$5^{\prime}$ - CCC TAT TAC CCT TGC CGA CCC TAC CTA CCT ACC CTG CGA TAC CTA AGA TGT GAA GTG AAT GAT GAG - $3^{\prime}$

$5^{\prime}$ - CCG ACC CTA CCT ACC TAC CCT GCG ATG ATG TGA A - 3'

$5^{\prime}$ - CCG ACC CTA CCT ACC TAC CCT GCG ATG ATA GCA T - 3'

$5^{\prime}$ - CCG ACC CTA CCT ACC TAC CCT GCG ATT TAG GTT T - 3' 


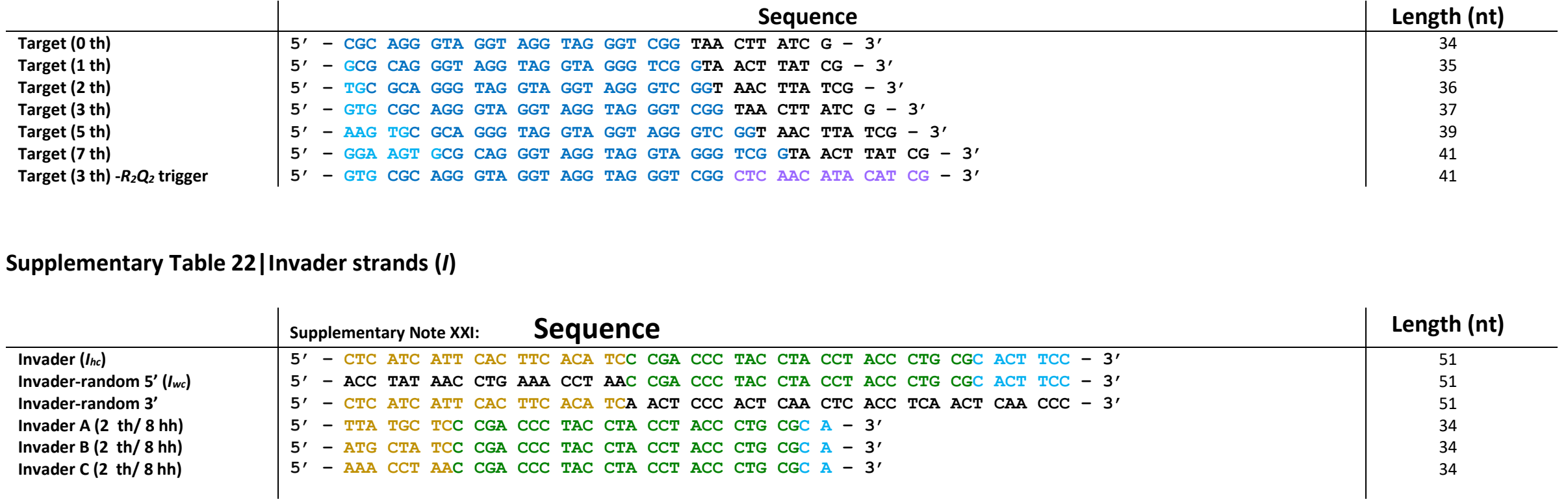

\section{Supplementary Table 23|Reporter strands}

\begin{tabular}{|c|c|c|}
\hline & Sequence & Length (nt) \\
\hline Reporter $1(R)$ & $5^{\prime}$ - AGG GTC GGC AAG GGT AAT AGG GAC ttt-Cy3 - 3' & 27 \\
\hline Reporter 1 quencher (Q) & 5' - IowaBlackFQ-ttt GTC CCT ATT ACC CTT GCC - 3' & 21 \\
\hline $\operatorname{Rep}_{A}\left(R_{2}\right)$ & $5^{\prime}$ - Cy5-ttt CGA TGT ATG TTG AGG CGT GCC GTT TTC GGC ACG CGA TGT GAA - 3' & 45 \\
\hline Rep $\mathrm{A}_{\mathrm{Auencher}}\left(Q_{2}\right)$ & $5^{\prime}$ - CAA CAT ACA TCG ttt-IowaBlackRQ - 3' & 15 \\
\hline Rep fluorescence & 5' - RhodamineRed ${ }^{\text {TMX}} \mathrm{X}$-ttt GTC GAT GTA TGT TGA GGC GTG CCG TTT TCG GCA CGC GAT AGC AT - 3' & 47 \\
\hline Rep в quencher & $5^{\prime}$ - CAA CAT ACA TCG AC ttt-BlackHoleQuencher2 & 17 \\
\hline Rep fluorescence & 5' - AlexaFluor ${ }^{\circledR}$ 48-ttt GTC GAT GTA TGT TGA GGC GTG CCG TTT TCG GCA CGC TTA GGT TT - 3' & 47 \\
\hline Repc quencher & $5^{\prime}$ - CAA CAT ACA TCG AC ttt-IowaBlackFQ - 3' & 17 \\
\hline
\end{tabular}


1. SantaLucia, J., Jr. \& Hicks, D. The Thermodynamics of DNA Structural Motifs. Annu Rev Biophys Biomol Struct 33, 415-440 (2004).

2. Koehler, R.T. \& Peyret, N. Thermodynamic Properties of DNA Sequences: Characteristic Values for the Human Genome. Bioinformatics 21, 33333339 (2005).

3. Zhang, D.Y. \& Winfree, E. Control of DNA Strand Displacement Kinetics Using Toehold Exchange. Journal of the American Chemical Society 131, 17303-17314 (2009).

4. Liu, W.-T., Wu, J.-H., Li, E.S.-Y. \& Selamat, E.S. Emission Characteristics of Fluorescent Labels with Respect to Temperature Changes and Subsequent Effects on DNA Microchip Studies. Applied and environmental microbiology 71, 6453-6457 (2005).

5. Moreira, B.G., You, Y., Behlke, M.A. \& Owczarzy, R. Effects of Fluorescent Dyes, Quenchers, and Dangling Ends on DNA Duplex Stability. Biochem Biophys Res Commun 327, 473-484 (2005).

6. Lagarias, J.C., Reeds, J.A., Wright, M.H. \& Wright, P.E. Convergence Properties of the Nelder-Mead Simplex Method in Low Dimensions. SIAM J. Optim. 9, 112-147 (1998). 